\title{
Resonance Production in \\ Two-PHOTON INTERACTIONS ${ }^{\star}$
}

\author{
Natalie Ann Roe \\ $-$ \\ Stanford Linear Accelerator Center \\ Stanford University \\ Stanford, California 94309
}

February 1989

Prepared for the Department of Energy

under contract number DE-AC03-76SF00515

Printed in the United States of America.-Available from the National Technical Information Service, U.S. Department of Commerce, 5285 Port Royal Road, Springfield, Virginia 22161. Price: Printed Copy A06, Microfiche A01.

* Ph.D. Dissertation 


\begin{abstract}
Resonance production in two-photon interactions is studied using data collected with the ASP detector at the PEP $e^{+} e^{-}$storage ring located at the Stanford Linear Accelerator Center.

The ASP detector is a non-magnetic lead-glass calorimeter constructed from 632 lead-glass bars. It covers $94 \%$ of $4 \pi$ in solid angle, extending to within $20^{\circ}$ of the beamline. Lead-scintillator calorimeters extend the coverage to within $21 \mathrm{mr}$ of the beamline on both-sides. Energy resolution of $\frac{10 \%}{\sqrt{E}}$, where $E$ is the energy is $\mathrm{GeV}$, is achieved for electrons and photons in the lead-glass calorimeter, and particle trajectories are reconstructed with high efficiency. A total luminosity of $108 \mathrm{pb}^{-1}$ was collected with the ASP detector at a center-of-mass energy of $29 \mathrm{GeV}$.

The observed process is $e^{+} e^{-} \rightarrow e^{+} e^{-} \gamma^{*} \gamma^{*} \rightarrow e^{+} e^{-} X$, where $\mathrm{X}$ is a pseudoscalar resonance $\left(J^{P C}=0^{-+}\right)$and $\gamma^{*}$ is a virtual (mass $\neq 0$ ) photon. The outgoing electrons scatter down the beampipe and are not detected. The observed resonances are the $\eta$ and $\eta^{\prime}$ mesons, with masses of 549 and $958 \mathrm{MeV}$, respectively. They are detected in the $\gamma \gamma$ decay mode; a total of $2380 \pm 49 \eta \rightarrow \gamma \gamma$ and $568 \pm .26 \eta^{\prime} \rightarrow \gamma \gamma$ events are observed.
\end{abstract}

From the number of events, the detection efficiency, and the calculated production cross sections the radiative widths, $\Gamma_{\gamma \gamma}$, of the $\eta$ and $\eta^{\prime}$ were measured and found to be:

$$
\begin{aligned}
\Gamma_{\gamma \gamma}(\eta) & =.481 \pm .010 \pm .047 \mathrm{keV} \\
\Gamma_{\gamma \gamma}\left(\eta^{\prime}\right) & =4.71 \pm .22 \pm .70 \mathrm{keV}
\end{aligned}
$$

These results are in good agreement with the world average values.

The radiative width is a probe of the quark content, because photons couple to the quark charge. In the framework of flavor SU(3) symmetry of the light (up, down, strange) quarks, the pseudoscalar mixing angle gives the quark content of the $\eta$ and $\eta^{\prime}$ mesons. Based on the radiative widths quoted above, the pseudoscalar mixing angle has been determined to be $\theta_{p}=-19.8 \pm 2.5^{\circ}$, in agreement with results from $\mathrm{J} / \psi$ decays. 


\section{Acknowledgments}

I would like to take this opportunity to say a word of thanks. First, to my advisor David Burke, for his advice and support, his boundless energy, and his enthusiasm for physics.

Many thanks are also due to the members of the ASP collaboration, who made this experiment the success it has been and who have been a pleasure to work with. In particular, I acknowledge the camaraderie of my office-mate, Chris Hawkins, who was a ready source of Fortran wit and wisdom. Tom Steele's valuable friendship and TEX-pertise smoothed the watcrs and made the journey much more pleasant. Thanks also to Lydia Beers, who brightened the way with humor and a friendly smile.

Finally, I want to thank my mother for weekly doses of encouragement, my father and brothers for keeping my Alfa Romeo running, and my husband Michael for everything else. 


\section{Contents}

Abstract $\quad$ iii

Acknowledgments $\quad$ iv

List of tables $\quad$ vii

List of figures viii

1 Introduction to Two-Photon Interactions 1

1.1 Historical Development

1.2 The Equivalent Photon Approximation 6

1.3 Properties of Two-Photon Interactions 7

1.4 Exact Lowest-Order Calculations 10

1.5 The Pseudoscalar Resonances and SU(3) Symmetry 13

2 Experimental Apparatus 16

2.1 The ASP Detector $\quad$ - 17

2.2 Online Calibration and Monitoring 23

2.3 The Trigger 25

3 Event Reconstruction 30

3.1 Off-line Calibration 30

3.2 Energy Determination 32

3.3 Tracking $\quad 35$

4 Event Simulation $\quad 39$

4.1 Event Generation $\quad 40$

4.2 Detector Simulation 41

4.3 Trigger Simulation 45

5 Event Selection $\quad 48$

5.1 Production First Pass 48

5.2 Analysis First Pass $\quad 49$ 
5.3 Final Event Selection 53

6 Background Calculations $\quad 57$

6.1 Sources of Background Events 57

6.2 Fourth-Order QED Backgrounds $\quad 57$

6.3 Two-photon backgrounds $\quad 60$

6.4 Cosmic Rays $\quad$. 63

6.5 Beam-Gas Interactions _ _ 64

6.6 Summary of Background Contributions 68

7 Efficiency Calculations $\quad 70$

7.1 Detector Acceptance 71

7.2 Trigger Efficiency - - 74

$\begin{array}{lll}7.3 & \text { Production First Pass Efficiency } & 78\end{array}$

$\begin{array}{ll}7.4 \text { Analysis First Pass Efficiency } & 79\end{array}$

7.5 Final Event Selection Efficiency $\quad 80$

7.6 Luminosity Measurement $\quad 85$

8 Results and Conclusions $\quad 88$

8.1 Fitting the $\gamma \gamma$ Invariant Mass Distribution $\quad 88$

8.2 The Radiative Widths of the $\eta$ and $\eta^{\prime}$. $\quad 91$

8.3 The Pseudoscalar Mixing Angle 95

8.4 Summary and Conclusions $\quad 98$

$\begin{array}{llr}\text { Appendix A } & \text { Forward Drift Chambers } & 99\end{array}$

A.1 Mechanical Construction $\quad 100$

A.2 Operation and Electronic Read-Out 101

$\begin{array}{llr}\text { A.3 Performance } & 102\end{array}$

REFERENCES 104 


\section{Tables}

1.1 Spin $1 / 2$ Fundamental Particles 3

1.2 Mediators of the Fundamental Forces. 3

1.3 Definition of Frequently Used Symbols $\quad 8$

1.4 Neutral Members of the Pseudoscalar Nonet 14

2.1 Trigger Definitions 27

4.1 $Q^{2}$ Dependence of Two-Photon Cross Sections 41

6.1 Summary of Background Contributions, 69

7.1 Detector acceptance for $\eta$ and $\eta^{\prime}$ events 71

$7.2 \eta$ peak and width measurements 76

$\begin{array}{lll}7.3 & \text { Efficiency of production first pass cuts } & 79\end{array}$

$\begin{array}{lll}7.4 & \text { Efficiency of analysis first pass cuts } & 79\end{array}$

7.5 Efficiency of final event selection cuts 81

7.6 Efficiency of analysis on data and simulated radiative Bhabhas 82

7.7 Summary of efficiencies.

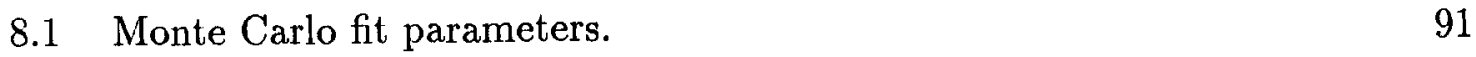

8.2 Fit parameters for $\gamma \gamma$ invariant mass distribution. $\quad 92$

8.3 Summary of quantities for calculation of radiative width. 93

8.4 Summary of measurements of the $\eta$ radiative width. 94

8.5 Summary of measurements of the $\eta^{\prime}$ radiative width. $\quad 95$ 


\section{Figures}

1.1 Photon-Photon Scattering 2

1.2 Two-Photon Production of electron pairs 6

1.3 Kinematics of the Two-Photon Process 7

2.1 Side view of the $\overline{\mathrm{A}} \mathrm{SP}$ detector $\quad 18$

2.2 Cross section of the central detector 19

2.3 Forward Shower Counter conștruction 24

2.4 Summing Circuit Diagram - 26

2.5 Trigger efficiency vs. lead-glass energy. 28

3.1 Attenuation in the lead glass 31

3.2 Radiation length of the lead glass vs. $\phi$. 33

3.3 Resolution in the lead glass 35

3.4 Resolution on Intercept $\quad 38$

4.1 Ratio of simulated lead-glass signal to data. 43

4.2 Ratio of simulated central PWC signal to data. 44

5.1 Threshold behavior of 'super trigger'. $\quad 50$

5.2 Lead-Glass quadrant time. $\quad 52$

5.3 Lead-Glass time vs. TOF time. $\quad 52$

5.4 Lead-Glass time - TOF time.

5.5 Shower shape of photons and $\pi^{\circ}$ 's. $\quad 55$

$5.6 \mathrm{p}_{\mathrm{t}}$ distribution of final data sample. $\quad 56$

5.7 Invariant mass distribution of final data sample. $\quad 56$

6.1 Feynman diagrams for $e^{+} e^{-} \rightarrow\left(e^{+} e^{-}\right) \gamma \gamma$

$6.2 e^{ \pm} \gamma$ background invariant mass $\quad 60$

$\begin{array}{lll}6.3 & f_{2}(1270) \text { background invariant mass } & 62\end{array}$ 
6.4 Crystal Ball $\pi^{\circ} \pi^{\circ}$ invariant mass distribution 63

6.5 Continuum $\pi^{\circ} \pi^{\circ}$ background contribution $\quad 63$

6.6 Timing distribution of identified cosmic ray events 65

6.7 Timing distribution of final sample. $\quad 65$

6.8 Z distributions of $\eta$ events. $\quad 67$

6.9 Invariant mass of beam-gas events. 68

$\begin{array}{lll}7.1 \theta \text { distribution for } \eta \text { events. } & 72\end{array}$

$7.2 \theta$ distribution for $e^{+} e^{-} \rightarrow\left(e^{+} e^{-}\right) e^{+} e^{-}$events. 74

7.3 Raw energy distribution, Monte Carlo vs. data, for e-pairs. 77

7.4 Central PWC efficiency vs. track energy. $\quad 83$

7.5. Stray energy distribution for Monte Carlo and data. 85

7.6 Lead glass shower shape distributions.

7.7 Central PWC Shower shape distributions. $\quad 86$

$\begin{array}{lll}7.8 & \text { Angular distribution of Bhabha events. } & 87\end{array}$

8.1 Invariant Mass of Monte Carlo $\eta$ events. $\quad 89$

8.2 Invariant Mass of Monte Carlo $\eta^{\prime}$ events. 90

8.3 Invariant Mass of Monte Carlo $f_{2}(1270)$ events. $\quad$ : 90

$\begin{array}{llr}8.4 & \text { Fitted } \gamma \gamma \text { Invariant Mass. } & 92\end{array}$

A.1 Resolution of forward drift chambers. 103

$\begin{array}{lll}\text { A.2 Drift velocity vs. distance from sense wire. } & 104\end{array}$ 


\section{Introduction to Two-Photon Interactions}

This thesis is a study of the production of $\eta$ and $\eta^{\prime}$ mesons in photon-photon interactions. Time-reversal invariance implies that any state produced by two photons may also decay back into two photons, and these are in fact the reactions which have been observed:

$$
\gamma \gamma \rightarrow \eta \rightarrow \gamma \gamma \text { and } \gamma \gamma \rightarrow \eta^{\prime} \rightarrow \gamma \gamma
$$

By way of introduction, let us first briefly describe the nature of two-photon interactions and the role of mesons in particle physics.

The interaction of two photons is a purely quantum effect which was first understood in the early 1930's with the advent of Quantum Field Theory. ${ }^{(1)}$ According to the laws of classical electrodynamics, photons do not interact; instead, their electromagnetic fields add linearly. This principle of the linear superposition of electromagnetic fields is well established in the macroscopic domain. ${ }^{(2)}$ However at the subatomic level there is a finite probability for two photons to scatter, either elastically, into two photons, or inelastically, producing a pair of charged particles. These processes are illustrated in the Feynman diagrams of fig. 1.1.

Because photons couple only to electric charge, they cannot couple directly to one another. The photon self-interaction occurs by virtue of the Heisenberg uncertainty principle, which allows a photon to become, for a short time, a pair of virtual charged particles. This fluctuation of a photon into two charged particles is highly improbable unless the photon has an energy greater than twice the mass of the lightest charged 

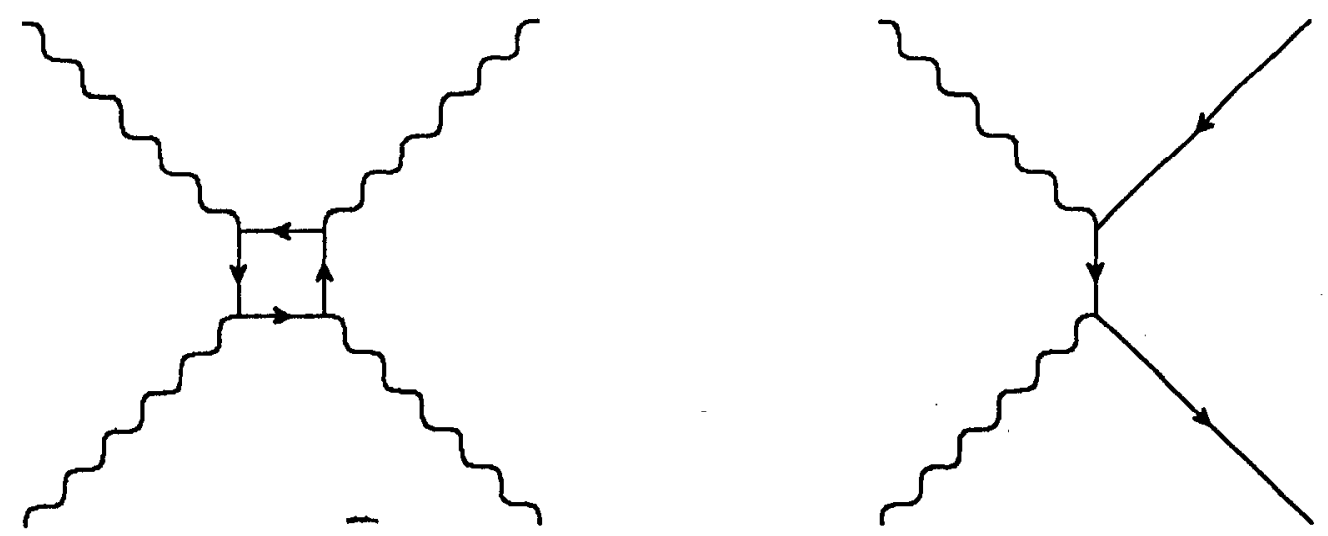

Figure 1.1. The scattering of two photons, a) elastically into two photons, and b) inelastically into a pair of charged particles.

particle, the electron. Photons of this energy are gamma rays with a frequency greater than $10^{20}$ cycles/sec. Therefore it is not surprising that the scattering of photons by photons is not an everyday feature! To reach the regime where we can observe this process we need particle accelerators where such high energies are available. The data for this research were accumulated with the ASP detector at the PEP $e^{+} e^{-}$storage ring $(\sqrt{s}=29 \mathrm{GeV})$, located at the Stanford Linear Accelerator Center.

Mesons, such as the $\eta$ and $\eta^{\prime}$, are quark anti-quark pairs, which we denote by $\mathrm{q} \overline{\mathrm{q}}$. Quarks are fundamental particles, and together with the leptons, they are the building blocks of all matter. Quarks are the constituents of protons and neutrons, from which atomic nuclei are made. The leptons include the familiar electron, and its associated neutrino, which form a doublet. There are two other known lepton doublets, or 'generations' of leptons. The quarks are also arranged in doublets, each doublet consisting of one quark with $+\frac{2}{3}$ of the electron charge and one with $-\frac{1}{3}$ of the electron charge. The three generations of leptons matches the three known generations of quarks. (We don't know if there is something special about the number three, or if there are additional generations yet to be discovered.) All of the known quarks and leptons are given in table 1.1. In addition to quarks and leptons, which are spin $1 / 2$ fermions, the list of fundamental particles includes some integer-spin bosons which are the mediators of the four fundamental forces. These include the photon 
for the electromagnetic force, the $W^{ \pm}$and $Z^{0}$ for the weak force, and gluons for the strong force, all particles with spin one. The as yet undiscovered graviton for the gravitational force is predicted to have spin two. These particles are summarized in table 1.2. (Particles which are anticipated but not yet discovered are in parentheses.)

Table 1.1. Spin 1/2 Fundamental Particles.

\begin{tabular}{|c|c|c|c|c|}
\hline \multicolumn{5}{|c|}{ Spin 1/2 Fundamental Particles } \\
\hline \multicolumn{3}{|c|}{ Charge } \\
\hline \multirow{2}{*}{ Quarks } & up & charm & (top) & $+\frac{2}{3}$ \\
& down & strange & bottom & $-\frac{1}{3}$ \\
\hline \multirow{2}{*}{ Leptons } & electron & muon & tau & -1 \\
& $\nu_{e}$ & $\nu_{\mu}$ & $\nu_{\tau}$ & 0 \\
\hline
\end{tabular}

Table 1.2. Mediators of the Fundamental Forces.

\begin{tabular}{|c|c|}
\hline Force & Mediator \\
\hline Electromagnetism & photon \\
Weak Force & $W^{ \pm}, Z^{\circ}$ \\
Strong Force & gluons \\
Gravity & (graviton) \\
\hline
\end{tabular}

Quarks come in several different 'flavors'; five have been discovered so far, called up, down, charm, strange and bottom, and it is hoped that the sixth, the top quark, will soon be discovered. In addition, each quark flavor comes in three different 'colors', which represent additional degrees of freedom. Although quarks are fractionally charged, they always appear in combinations which have integer charge. For example, a meson consisting of a $q \bar{q}$ pair will have charge $0,+1$ or -1 , because anti-quarks carry the opposite chargc of the quarks. 
When two photons interact through the creation of virtual quark pairs, the quarks may strongly interact in the final statc to producc a $q \bar{q}$ bound state, or meson. This process is resonant when the invariant mass of the photons is close to that of the produced meson, greatly enhancing the rate. Any meson produced in a two-photon interaction will, of course, be electrically neutral. The $\eta$ and the $\eta^{\prime}$ are neutral mesons composed primarily of the light quarks, $\mathrm{u}, \mathrm{d}$ and $\mathrm{s}$ (for up, down and strange). In fact, we will see later that the $\eta$ and the $\eta^{\prime}$ can be described as linear combinations of $u \bar{u}, d \bar{d}$, and $s \bar{s}$ quark-pairs. In this study we will measure the coupling of the $\eta$ and $\eta^{\prime}$ to two photons, which is called the radiative width, by observing the rate at which each is produced in two-photon interactions. This will allow us to determine how much of each quark flavor each meson contains, because the photon coupling to quarks goes like the fourth power of the quark charge; therefore photons couple more strongly to up quarks than to down or strange quarks.

\subsection{Historical Development}

The interaction of two photons was first described by Landau and Lifshitz ${ }^{(1)}$ in 1934. Shortly thereafter, Euler and Kockel ${ }^{(3)}$ calculated the cross section for the elastic scattering of light by light, $\gamma \gamma \rightarrow \gamma \gamma$, and Breit and Wheeler ${ }^{(4)}$ calculated the cross section for the inelastic scattering process $\gamma \gamma \rightarrow e^{+} e^{-}$. Intense beams of highly energetic photons are required to produce a measurable rate, and consequently the observation of these processes remained experimentally unattainable for several decades.

The first observations of photon-photon scattering employed a technique proposed in 1951 by Primakoff( ${ }^{(5)}$. An energetic photon beam was used on a nuclear target, such as a sheet of copper or lead. The real photons in the beam interacted with the virtual photons in the Coulombic field of the nuclei. The advantage of this method over using two beams of photons directed at each other is the high density of virtual photons in the target. 'I'he first measurement of the radiative width of the $\eta$ was performed using this technique in 1967 by Bemporad et al. ${ }^{(6)}$. However Primakoff production suffers from a serious experimental difficulty due to a background from 
the interaction of the incident photon beam with the hadronic field of the nucleus. To sort out the electromagnetic and hadronic production, the different dependence of the cross sections on nuclear species, incident energy, and production angle is exploited in a global fit. However, the systematic uncertainties can be large in such a fit.

It was Low ${ }^{(7)}$ who first suggested, in 1960, that resonance production in photonphoton interactions could be studied at $e^{+} e^{-}$storage rings. Specifically, he proposed this as a means of measuring the radiative width of the $\pi^{\circ}$ meson, which was known only to three orders of magnite at that time. At an $e^{+} e^{-}$storage ring, the electron and positron beams are accompanied by beams of virtual photons, making them sources of high energy photon-photon collisions. That the electromagnetic field of a relativistic charged particle can be viewed as a collection of virtual photons was first realized in the 1930's by Williams ${ }^{(8)}$ and Weizsäcker ${ }^{(9)}$, who calculated the resulting spectrum of virtual photons in the approximation that the electron energy was much greater than its mass. Low used the 'equivalent photon approximation' (EPA) of Williams and Weizsäcker for the photon flux in an $e^{+} e^{-}$storage ring and calculated the production rate for the process $e^{+} e^{-} \rightarrow e^{+} e^{-} \pi^{\circ}$.

When the first $e^{+} e^{-}$storage rings were brought into operation in the early 1960 's, two-photon interactions attracted little attention because the energies and luminosities were too low for such processes to be observed. Instead, attention was focused on the states produced via the one-photon annihilation channel, which is favored at low beam energies over the two-photon process.

Not until 1970 were two-photon reactions detected, at Novosibirsk ${ }^{(10)}$ with the VEPP-2 ring and at Frascati(11) with the Adone ring. The process $e^{+} e^{-} \rightarrow e^{+} e^{-} \gamma \gamma \rightarrow$ $e^{+} e^{-} e^{+} e^{-}$, shown in fig. 1.2, was the first to be observed at both machines. This process is exactly calculable using the theory of Quantum Electrodynamics, or QED, and it is useful as a calibration process as we will see later.

The first observation of resonance production in two-photon interactions did not occur until 1979, when the MarkII detector on the SPEAR storage ring at SLAC reported production of the $\eta^{\prime}$ meson in two-photon interactions. Since that time the radiative widths of several other mesons have been measured using the tech- 


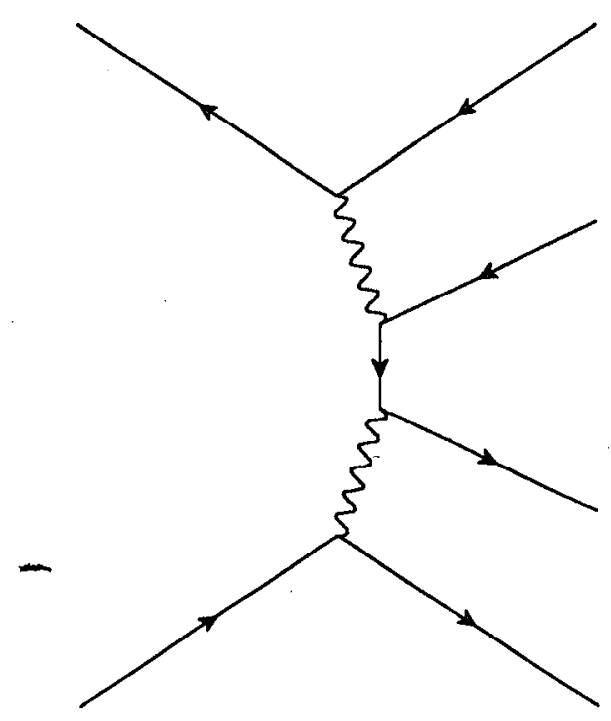

Figure 1.2. Electron pair production in two-photon interactions.

nique proposed by Low. These measurements are experimentally challenging, and in fact the radiative width of the $\pi^{\circ}$ has only recently been measured in two photon interactions $^{(12)}$.

\subsection{The Equivalent Photon Approximation}

In fig. 1.3 the formation of a resonance by two virtual photons is illustrated, and the kinematic variables are defined in table 1.3. Following Low, we will calculate the cross section for this process in the equivalent photon approximation (EPA) of Williams and Weizsäcker. Exact treatments are available and have been used in this analysis, but for an intuitive understanding EPA provides a very useful description.

In EPA the problem is divided into two parts, the emission of the photons and their subsequent interaction. The energy spectrum of the bremsstrahlung photons is given by:

$$
\frac{d N}{d \omega}=\frac{N(\omega)}{\omega} ; \quad N(\omega) \sim \frac{2 \alpha}{\pi} \ln \frac{E}{m_{e}}
$$

The total cross section for the reaction $\gamma \gamma \rightarrow X$ is a function only of the invariant mass of the $\gamma \gamma$ system, $s \sim 4 \omega_{1} \omega_{2}$, so we can write the cross section in the form:

$$
\sigma\left(e^{+} e^{-} \rightarrow e^{+} e^{-} X\right)=\int \frac{d \omega_{1}}{\omega_{1}} \int \frac{d \omega_{2}}{\omega_{2}} N\left(\omega_{1}\right) N\left(\omega_{2}\right) \sigma_{\gamma \gamma \rightarrow X}(s)
$$




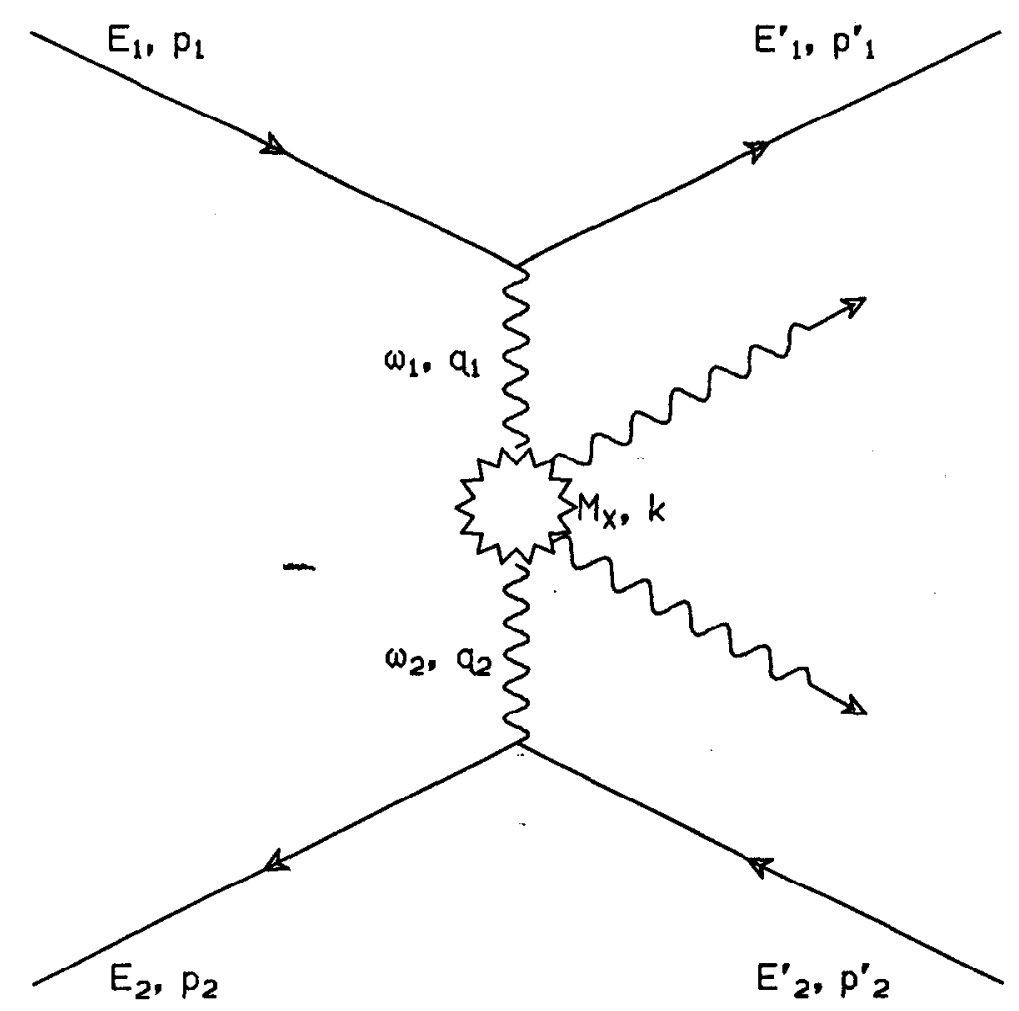

Figure 1.3. Resonance production in two-photon interactions, with the notation for the variables used.

Substituting for $N(\omega)$ using eq. 1.1 and keeping only the leading terms in $\ln \left(\frac{E}{m_{e}}\right)$ this becomes

$$
\sigma\left(e^{+} e^{-} \rightarrow e^{+} e^{-} X\right) \sim 2 \frac{\alpha 2}{\pi}\left(\ln \frac{E}{m_{e}}\right)^{2} \int \frac{d s}{s} \sigma_{\gamma \gamma \rightarrow X}(s) f\left(\frac{\sqrt{s}}{2 E}\right)
$$

where $\mathrm{f}$ is a function given by

$$
f(x)=-\left(2+x^{2}\right)^{2} \ln x-\left(1-x^{2}\right)\left(3+x^{2}\right)
$$

which takcs on values ranging from 1 to 10 for most problems of interest.

\subsection{Properties of Two-Photon Interactions}

From eq. 1.3 several important properties of two-photon interactions can be seen at once. First, the cross section rises logarithmically with increasing beam energy. This is in marked contrast to annihilation into one photon, which falls like the inverse 
Table 1.3. Definition of Frequently Used Symbols.

\begin{tabular}{|c|c|}
\hline Symbol & Description \\
\hline$m_{e}$ & Electromagnetic coupling constant \\
$\theta, \phi$ & Electron mass \\
$\mathrm{p}_{\mathrm{t}}$ & Component of momentum transverse to $e^{ \pm}$beams \\
$\mathrm{E}, \mathrm{p}$ & Electron beam energy, 4-vector \\
$\mathrm{E}^{\prime}, \mathrm{p}^{\prime}$ & Scattered electron beam energy, 4-vector \\
$\omega, \mathrm{q}$ & Photon energy, 4-vector \\
$s=4 \omega_{1} \omega_{2}$ & Invariant mass of $\gamma \gamma$ system \\
$Q^{2}$ & Negative photon mass squared \\
$\mathrm{X}$ & System produced in $\gamma \gamma$ interaction \\
$M_{X}, k$ & Mass, 4-vector of $\mathrm{X}$ \\
$\Gamma_{\gamma \gamma}^{X}$ & Radiative width for $X \rightarrow \gamma \gamma$ \\
$\Gamma^{X}$ & Total width of $\mathrm{X}$ \\
\hline
\end{tabular}

of the beam energy squared. This explains the remarkable fact that the two-photon process dominates over the annihilation channel at beam energies greater than a few $\mathrm{GeV}$, despite being higher order in $\alpha$. However, the produced state frequently has a large longitudinal boost, pushing the final state particles to small angles and reducing the experimental acceptance. Because the photons can have any energy up to the beam energy, two-photon interactions automatically scan the mass region from twice the electron mass up to twice the beam energy. In a world of finite luminosity and running time one is in practice limited to lower masses because of the $\frac{1}{s}$ decrease in photon flux.

Another important feature which is central to EPA is the small scattering angle of the outgoing electrons, $\theta_{e} \sim \sqrt{\frac{m_{e}}{E}}$. This has important experimental consequences, since most of the time both electrons escape down the beampipe and are not detected. 
In the 'single-tag' case, where the experimenter requires one of the electrons to be detected above a minimum tagging angle, typically $\sim 1^{\circ}$, the cross section is reduced by about an order of magnitude. Double-tagged experiments are down by about two orders of magnitude in signal and consequently very few have been performed.

The small scattering angle implies a small mass for the virtual photons, defined by:

$$
q^{2}=\left(p^{\prime}-p\right)^{2} \sim-2 E E^{\prime}\left(1-\cos \theta^{\prime}\right)
$$

Here $\theta^{\prime}$ is the angle of the scattered electron and is typically very close to zero. The virtual photons are space-like, i.e.they have a negative mass. They are often referred to as 'quasi-real', being almost massless like a real photon. The variable most often used is the positive quantity $Q^{2}=-q^{2}$. In events with high $Q^{2}$, such as single- or double-tagged events, the EPA is no longer a good approximation. In this case a form factor should be used which incorporates the $Q^{2}$ dependence.

It is straightforward to identify the process $e^{+} e^{-} \rightarrow e^{+} e^{-} \gamma \gamma \rightarrow e^{+} e^{-} X$, even in the untagged case. The electrons are close to the beam-line and carry little transverse momentum. Conservation of momentum requires that the produced system $\mathrm{X}$ also have low net $p_{t}$. The maximum $p_{t}$ is given by

$$
\mathrm{p}_{\mathrm{t}}^{\max }=2 E \sin \theta_{v}
$$

where $\theta_{v}$ is the veto angle below which the electrons are undetected in the apparatus. Because usually only one electron will be scattered at a non-zero angle, most events will have a maximum net $p_{t}$ which is one-half this value. The longitudinal momentum will usually be non-zero, so that an isotropic angular distribution in the photonphoton center of mass will be peaked at lower angles in the lab frame. If $\mathrm{X}$ is a two-particle final state, such as $\gamma \gamma \rightarrow e^{+} e^{-}$, or decays to one as in the process $\gamma \gamma \rightarrow \eta \rightarrow \gamma \gamma$, the final state particles will be back-to-back in the plane transverse to the beam but will usually be acollinear in the plane containing the beam. This topology, together with a low net $p_{t}$ and an observed energy much less than twice the beam energy, is a very distinctive signature for two-photon interactions. 


\subsection{Exact Lowest-Order Calculations}

Applying the Feynman rules ${ }^{(13)}$ to the diagram of fig. 1.3 gives the amplitude for the process $e^{+} e^{-} \rightarrow e^{+} c^{-} X:^{*}$

$$
M=-e^{2} \bar{u}\left(p_{1}^{\prime}\right) \gamma^{\mu} u\left(p_{1}\right) \bar{v}\left(p_{2}^{\prime}\right) \gamma^{\nu} v\left(p_{2}^{\prime}\right) \frac{1}{q_{1}^{2} q_{2}^{2}} T_{\mu \nu}
$$

In this expression, $u$ and $v$ are the Dirac spinors for the electron and positron, each of the photon propagators contributes a factor of $\frac{1}{q^{2}}$, and $T_{\mu \nu}$ is the amplitude for the process $\gamma \gamma \rightarrow X$. The cross section is then given by:

$$
d \sigma_{e^{+} e^{-} \rightarrow e^{+} e^{-} X}=\sum_{s p i n s}\left|M^{2}\right| \frac{(2 \pi)^{4} \delta\left(q_{1}+q_{2}-k\right) d \Gamma}{4 \sqrt{\left(p_{1} p_{2}\right)^{2}-m_{e}^{4}}} \frac{1}{(2 \pi)^{6}} \frac{d^{3} p_{1}^{\prime}}{2 E_{1}} \frac{d^{3} p_{2}^{\prime}}{2 E_{2}}
$$

where

$$
d \Gamma=\prod_{j} \frac{d^{3} k_{j}}{2 k_{j}^{0}(2 \pi)^{3}}
$$

is the Lorentz invariant phase space volume of the produced state $\mathrm{X}$ decaying into the final state particles $k_{j}$.

Following V. Budnev et al. ${ }^{(14)}$, we can define an unnormalized density matrix for the photon flux at the $i^{\text {th }}$ vertex by:

$$
\rho_{i}^{\mu \nu}=-\frac{1}{q_{i}^{2}} t_{i}^{\mu \nu} .
$$

The leptonic tensor $t^{\mu \nu}$ is obtained from eq. 1.8 by averaging over the initial state spins and summing over the final state spins (assuming unpolarized beams), giving a term for each $e^{ \pm} \gamma$ vertex of the form:

$$
\begin{aligned}
t_{i}^{\mu \nu} & =\sum_{\text {spins }} \overline{\bar{u}}\left(p_{1}^{\prime}\right) \gamma^{\mu} u\left(p_{i}\right) \bar{u}\left(p_{i}\right) \gamma^{\nu} u\left(p_{i}^{\prime}\right) \\
& =\frac{1}{2} \operatorname{Tr}\left[\gamma^{\mu}\left(p_{i}+m_{e}\right) \gamma^{\nu}\left(p_{i}^{\prime}+m_{e}\right)\right] \\
& =q_{i}^{2} g^{\mu \nu}-2\left(p_{i}^{\mu} p_{i}^{\prime \nu}+p_{i}^{\prime}{ }_{i}^{\mu} p_{i}^{\nu}\right)
\end{aligned}
$$

* The discussion of this section is based on formulae presented in Refs. 14 and 16. 
where $g^{\mu \nu}$ is the metric tensor defined according to the convention of Bjorken and Drell. ${ }^{(15)}$ Neglecting terms of order $\frac{m_{e}}{E}$ we can now write the cross section in the following form:*

$$
d \sigma_{e^{+} e^{-} \rightarrow e^{+} e^{-} X}=\frac{\alpha^{2}}{2 \pi^{4}} \frac{1}{q_{1}^{2} q_{2}^{2}} \rho_{1}^{\mu \mu^{\prime}} \rho_{2}^{\nu \nu^{\prime}} W_{\mu^{\prime} \nu^{\prime}, \mu \nu} \frac{1}{s} \frac{d^{3} p_{1}^{\prime}}{2 E_{1}} \frac{d^{3} p_{2}^{\prime}}{2 E_{2}} .
$$

In eq. 1.12 the photon flux factors $\rho^{\mu \nu}$ are separated from the physics of the $\gamma \gamma \rightarrow X$ vertex, which is containcd in the rank 4 tensor

$$
\begin{aligned}
W_{a^{\prime} b^{\prime}, a b} & =\frac{1}{2} \int M_{* a^{\prime} b^{\prime}} M_{a b}(2 \pi)^{4} \delta\left(q_{1}+q_{2}-k\right) d \Gamma, \\
M_{a b} & =F\left(q_{1}^{2}, q_{2}^{2}\right) T_{\mu \nu} \epsilon_{1 a}^{\mu} \epsilon_{2 b}^{\nu} .
\end{aligned}
$$

This differential expression has been used to generate Monte Carlo events and to evaluate the total cross section for two-photon production of $\eta$ and $\eta^{\prime}$ mesons. The form factor $F\left(q_{1}^{2}, q_{2}^{2}\right)$ describes how the interaction of virtual photons differs from that of real photons, and must be determined experimentally. For events with large $Q^{2}$, the cross section is usually suppressed, i.e. $F<1$, and in the limit $q_{1}^{2}, q_{2}^{2} \rightarrow 0, F \rightarrow 1$.

The polarization vectors $\epsilon$ describe the helicity structure of the $\gamma \gamma$ interaction, and the indices $a, b$ take on the values,+- , and 0 . Conservation of helicity and parity at the $\gamma \gamma$ vertex implies a number of interesting properties. A detailed analysis is given in a review by Poppe; ${ }^{(16)}$ taken together these conservation laws are known as Yang's theorem, ${ }^{(17)}$ which states that all states with even spin and all states with odd spin and even parity (except $J=1$ ) may be produced in $\gamma \gamma$ interactions. This is very different from the annihilation channel, in which the allowed quantum numbers are limited to those of the photon, $J^{P C}=1^{--}$. Yang's theorem applies only in the limit of real photons, $q_{1}^{2}, q_{2}^{2} \rightarrow 0$. If one of the photons is highly virtual any spin-parity combination may be produced. In all cases the charge conjugation must be even. These properties makc two-photon interactions very well suited to the observation of

* In this approximation, $\left(p_{1} p_{2}\right)^{2}-m_{e}^{4} \sim\left(E_{1} E_{2}-\vec{p}_{1} \cdot \vec{p}_{2}\right)^{2} \sim\left(2 E_{1} E_{2}\right)^{2}=\left(\frac{s}{2}\right)^{2}$. Also, we use the convention $\alpha=\frac{e^{2}}{h c}$ and $\hbar=c=1$. 
$C=+$ resonances. In this analysis we are concerned with the neutral pseudoscalars, which have $J^{P C}=0^{-+}$.

The conservation laws also dictate which helicity combinations may produce a state of given spin and parity. For pseudoscalar mesons, all combinations with one or more longitudinal photons vanish exactly. In the untagged case, only $\sigma_{T T}$, the cross section for transverse photons survives and eq. 1.12 becomes:

$$
\begin{aligned}
d \sigma_{e^{+} e^{-} \rightarrow e^{\mp} e^{-} X} & =\frac{\alpha^{2}}{2 \pi^{4}} \frac{1}{q_{1}^{2} q_{2}^{2}} \frac{\sqrt{X}}{s} 4 \rho_{1}^{++} \rho_{2}^{++} \sigma_{T T} \frac{d^{3} p_{1}^{\prime}}{2 E_{1}} \frac{d^{3} p_{2}^{\prime}}{2 E_{2}} \\
\sigma_{T T} & =\frac{1}{4 \sqrt{X}} W_{++,++}
\end{aligned}
$$

Here $X$ is the Möller flux factor,

$$
X=\left(q_{1} q_{2}\right)^{2}-q_{1}^{2} q_{2}^{2}=\frac{1}{4}\left[s^{4}-2 s^{2}\left(q_{1}^{2}+q_{2}^{2}\right)+\left(q_{1}^{2}-q_{2}^{2}\right)\right]
$$

where $s=4 \omega_{1} \omega_{2}$ is the invariant mass of the $\gamma \gamma$ system. As $q_{1}^{2}, q_{2}^{2} \rightarrow 0, X \rightarrow s^{4} / 4$. In the limit of real photons, the result for $\sigma_{T T}$ is given by:

$$
\sigma_{T T}=8 \pi(2 J+1) \frac{M_{X}}{s} \frac{\Gamma^{X} \Gamma_{\gamma \gamma}^{X}}{\left(s^{2}-M_{X}^{2}\right)^{2}+\Gamma^{2} M_{X}^{2}}
$$

For narrow resonances, such as the $\eta$ or $\eta^{\prime}$, the Breit-Wigner can be replaced by a delta function.* The cross section is then a function of $\Gamma_{\gamma \gamma}^{X}$ and $M_{X}$ only:

$$
\sigma(\gamma \gamma \rightarrow X)=8 \pi^{2}(2 J+1) \frac{\Gamma_{\gamma \gamma}^{X}}{M_{X}}(2 J+1) \delta\left(M_{X}^{2}-s\right)
$$

* For a narrow resonance,

$$
\lim _{M \Gamma \rightarrow 0} \frac{M_{X} \Gamma}{\left(M_{X}^{2}-M_{\gamma \gamma}^{2}\right)^{2}+M_{X}^{2} \Gamma^{2}}=\pi \delta\left(M_{X}^{2}-M_{\gamma \gamma}^{2}\right)
$$


The branching ratios of all significant decay modes have been established for the $\eta$ and $\eta^{\prime}$ mesons in fixed-target experiments, so a measurement of the production rate in $\gamma \gamma$ interactions provides a measurement not only of the radiative width but also of the total width. This is important because the narrow total width of these states makes them difficult to measure directly from the line shape.

To tie this discussion back into the results of section 2 , we can substitute eq. 1.17 into the EPA formula (eq. 1.3) to obtain an approximation for two-photon resonance production given by:

$$
\sigma(e e \rightarrow e e X)=\frac{16 \alpha^{2}}{M_{X}^{3}} \Gamma_{\gamma \gamma}^{X}(2 J+1)\left(\ln \frac{E}{m_{e}}\right)^{2} f\left(\frac{M_{X}}{2 E}\right)
$$

where $\mathrm{f}$ is the function defined in eq. 1.4. This is precisely Low's result for $\pi^{\circ}$ production in photon-photon interactions.

\subsection{The Pseudoscalar Resonances and SU(3) Symmetry}

The form of $\Gamma_{\gamma \gamma}^{X}$, where $\mathrm{X}$ is a pscudoscalar, is fixed by Lorentz and gauge invariance to be of the form

$$
\Gamma_{\gamma \gamma}^{X}=\frac{M^{3}}{64 \pi} g_{p}^{2}
$$

where $g_{p}$ describes the coupling strength between two real photons and the constituent quarks in the meson. It is interesting to note that the factor of $M^{3}$ exactly cancels a similar factor in eq. 1.18. As a result, the total two-photon production cross section for pseudoscalars decreases rather slowly with mass according to the function $f$ defined in eq. 1.4 .

A neutral meson can be described as a linear combination of $q \bar{q}$ pairs:

$$
P=\sum_{i} a_{i}\left|\mathrm{q}_{\mathrm{i}} \overline{\mathrm{q}}_{\mathrm{i}}\right\rangle
$$

where the coefficients $a_{i}$ run over all quark flavors and are satisfy $\sum_{i} a_{i}^{2}=1$. Because photons couple to charge, it follows that $\mathrm{g}$ will be proportional to $\sum_{i} a_{i} e_{i}^{2}$, where $e_{i}$ is the quark charge as given in Table 1 . In the case of the light pseudoscalar 
mesons this coupling is described by the triangle anomaly calculation of Adler, Bell and Jackiw, ${ }^{(18)}$ who found

$$
g_{p}=\frac{\sqrt{2} \alpha N_{c}}{\pi f_{\pi}} \sum_{i} a_{i} e_{i}^{2}
$$

This is a QCD calculation in which all the non-perturbative parts are lumped together into the pion decay constant, which has been measured in charged pion decay to be $f_{\pi}=93 \mathrm{MeV}$, and $N_{c}=3$ is the number of colors. Putting $g_{p}$ back into eq. 1.19, the dependence of the raliative width on the fourth power of the quark charges is evident.

The experimental determination of the coefficients $a_{i}$ is the goal of this analysis. The theoretical framework is provided by the flavor symmetry of SU(3), in which the masses of the three lightest quarks, u, d, and s, are taken to be equal. There are $3 \otimes 3=8 \oplus 1$ different $\mathrm{q} \overline{\mathrm{q}}$ combinations which are possible, giving a nonet composed of an octet and a singlet. There are several different nonets, one for each spin-parity combination. The pseudoscalar nonet consists of the pions, $\left(\pi^{+}, \pi^{-}, \pi^{\circ}\right)$, the kaons $\left(K^{+}, K^{-}, K^{\circ}, \bar{K}^{\circ}\right)$, the $\eta$ and the $\eta^{\prime}$. The masses, widths and branching ratios of the neutral members of the pseudoscalar nonet are summarized in table 1.4 .

Table 1.4. Neutral members of the pseudoscalar nonet.

\begin{tabular}{|c|c|c|c|}
\hline Resonance & Mass $(\mathrm{MeV})$ & $\Gamma$ & $\operatorname{Br}(\gamma \gamma)$ \\
\hline$\pi^{\circ}$ & $134.9 \pm .004$ & $7.57 \pm 0.32 \mathrm{eV}$ & $98.8 \pm .03 \%$ \\
\hline$\eta$ & $548.8 \pm 0.6$ & $1.35 \pm .08 \mathrm{keV}$ & $38.9 \pm 0.4 \%$ \\
\hline$\eta^{\prime}$ & $957.6 \pm 0.3$ & $240 \pm 30 \mathrm{keV}$ & $2.23 \pm .18 \%$ \\
\hline
\end{tabular}

The SU(3) basis states for the neutral members of the pseudoscalar nonet are defined by:

$$
\begin{aligned}
& \left|\pi^{\circ}\right\rangle=\frac{1}{\sqrt{2}}|u \bar{u}-d \bar{d}\rangle \\
& \left|\eta_{8}\right\rangle=\frac{1}{\sqrt{6}}|u \bar{u}+d \bar{d}-2 s \bar{s}\rangle \\
& \left|\eta_{1}\right\rangle=\frac{1}{\sqrt{3}}|u \bar{u}+d \bar{d}+s \bar{s}\rangle
\end{aligned}
$$


These states differ from the physically observed $\eta$ and $\eta^{\prime}$ resonances, so to differentiate them we have uscd subscripts. The $\eta_{1}$ is the singlet state and the $\eta_{8}$ belongs to the octet. The observed mass eigenstates, are a mixture of $\eta_{1}$ and $\eta_{8}$, defined by the mixing angle $\theta_{p}$ :

$$
\begin{array}{r}
|\eta\rangle=\cos \theta_{p}\left|\eta_{8}\right\rangle-\sin \theta_{p}\left|\eta_{1}\right\rangle \\
\left|\eta^{\prime}\right\rangle=\sin \theta_{p}\left|\eta_{8}\right\rangle+\cos \theta_{p}\left|\eta_{1}\right\rangle
\end{array}
$$

To see if $\mathrm{SU}(3)$ symmetry is a reasonable approximation, let us compute the radiative width of the $\pi^{\circ}$ using the $\mathrm{SU}(3)$ quark content assignment and the AdlerBell-Jackiw calculation, eq. 1.23. The result, with

$$
\sum_{i} a_{i} e_{i}^{2}=\frac{1}{\sqrt{2}} e_{u}^{2}-\frac{1}{\sqrt{2}} e_{d}^{2}=\frac{1}{3 \sqrt{2}}
$$

is

$$
g_{\pi^{\circ}}=\frac{\alpha}{\pi f_{\pi}}
$$

Using eq. 1.21 results in $\Gamma\left(\pi^{\circ} \rightarrow \gamma \gamma\right)=7.6 \mathrm{eV}$, to be compared with the current experimental average $\Gamma\left(\pi^{\circ} \rightarrow \gamma \gamma\right)=7.5 \pm .2 \mathrm{eV}$.

For the $\eta$ and $\eta^{\prime}$ mesons a similar calculation yields

$$
\begin{aligned}
& \Gamma_{\gamma \gamma}^{\eta}=\frac{\alpha^{2}}{64 \pi^{3}} \frac{M_{\eta}^{3}}{3}\left[\frac{1}{f_{8}} \cos \theta_{p}-\frac{\sqrt{8}}{f_{1}} \sin \theta_{p}\right]^{2} \\
& \Gamma_{\gamma \gamma}^{\eta^{\prime}}=\frac{\alpha^{2}}{64 \pi^{3}} \frac{M_{\eta^{\prime}}^{3}}{3}\left[\frac{1}{f_{8}} \sin \theta_{p}+\frac{\sqrt{8}}{f_{1}} \cos \theta_{p}\right]^{2}
\end{aligned}
$$

As discussed further in chapter 8 , this result relating the radiative widths of the $\eta$ and $\eta^{\prime}$ mesons to the pseudo-scalar mixing angle can be used to determine $\theta_{p}$. The question of whether an admixture of other states, such as charm or gluons, could be present will also considered. 


\section{Experimental Apparatus}

The data for this experiment were collected at the Stanford Linear Accelerator Center (SLAC) on the PEP $e^{+} e^{-}$storage ring. SLAC is a national laboratory operated by Stanford University under contract from the U.S. Department of Energy, and is one of three national laboratories devoted to research in particle physics in the U.S.; the others are Fermilab, located near Chicago, Illinois, and Brookhaven National Lab, located in Brookhaven, New York. SLAC is located on a 480 -acre site adjacent to the Stanford University campus in Palo Alto, California. SLAC has been in operation since 1964, when construction of its two-mile linear electron accelerator was completed. This facility is the longest and most energetic linear electron accelerator available anywhere in the world today.

The PEP collider, an $e^{+} e^{-}$storage ring with a circumference of $2.2 \mathrm{~km}$, was constructed in 1980. Electrons and positrons for PEP are supplied at an energy of $14.5 \mathrm{GeV}$ by the two-mile linear accelerator. Three electron bunches and three positron bunches counter-rotate in the circular PEP vacuum pipe, colliding in six interaction regions (IR's) every $2.4 \mu \mathrm{s}$ at a total center-of-mass energy of $29 \mathrm{GeV}$. The peak luminosity achieved at PEP was approximately $3 \times 10^{31} \mathrm{~cm}^{-2} \mathrm{sec}^{-1}$, and the typical luminosity was about half that. Five of the six IR's were occupied by large, multi-purpose detectors. The sixth, known as IR 10, was initially uninstrumented.

In the spring of 1983 an experiment to search for weakly-interacting particles using photon tagging was proposed to be installed in IR 10 by D. Burke and R. 
Hollebeek. ${ }^{(19)}$ The basic idea was formulated in 1978 by $\mathrm{Ma}$ and $\mathrm{Okada}^{(20)}$, and further elaboratcd on in 1981 by Barbiellini, Richter and Siegrist. ${ }^{(21)}$ The idea is to tag the radiative process $e^{+} e^{-} \rightarrow \nu \bar{\nu} \gamma$ by detecting a single photon (the neutrinos have a very low cross section to interact in matter and escape undetected.) Counting the number of observed single photons determines the number of light neutrino families. Extending this idea, Burke and Hollebeek proposed to search for any particle which interacted weakly in matter, including exotic states such as supersymmetric particles. An inexpensive detector madofrom extruded lead-glass bars and optimized for photon detection was all that was required. The proposal was approved and work on the detector began immediately.

Assembly of the ASP detector was completed during the summer of 1984 and data-taking commenced that fall. The first data-taking cycle extended from November through May of 1984/85, and the second from November through February of 1985/86. The PEP ring was then shut down for an extended period while work on a new machine, the Stanford Linear Collider, took priority. The ASP delector was eventually removed from IR 10 and put into storage.

The ASP single-photon search ${ }^{(22)}$ yielded just one signal event, consistent with the expectations of the Standard Model for three generations of light neutrinos. Limits were placed on supersymmetric processes which could have contributed to the signal, and the number of light neutrino generations was limited to be less than 7.5 , at the $90 \%$ confidence level, the best limit available to date from an $e^{+} e^{-}$machine.

Designed to efficiently detect events with a single photon, the ASP detector was well suited to the detection of events with two photons in the final state, which is the signature required by this analysis. In this chapter the ASP detector, trigger and on-line monitoring systems are described, with emphasis on the lead-glass calorimeter and the trigger because they are most important for this analysis.

\subsection{The ASP Detector}

ASP was located in IR 10, the smallest interaction region at PEP, which was only accessible by entering a neighboring interaction region and walking down the 
PEP arcs. Another disadvantage was the location of the IR hall directly downstream from the $e^{-}$injection port, which resulted in high radiation levels during injection into PEP. Partially offsetting these disadvantages, IR 10 was $20 \mathrm{~m}$ underground. The earth shielded the detector, reducing the cosmic-ray flux by almost a factor of 3 .

The complete apparatus consisted of a lead-glass calorimeter and a system of forward detectors which together covered the solid angle without any gaps or cracks above a polar angle $\theta>21 \mathrm{mr}$. The detector is shown schematically in fig. 2.1 and fig. 2.2. The coordinaterystem has $+\hat{z}$ parallel to the beampipe (in the direction of the positron beam), $+\hat{y}$ vertically upwards, and $+\hat{x}$ pointing horizontally toward the center of the PEP ring. The polar coordinate system is conventionally defined with respect to these axes. In the following, each detector component is described, beginning with the beampipe and working outwards.

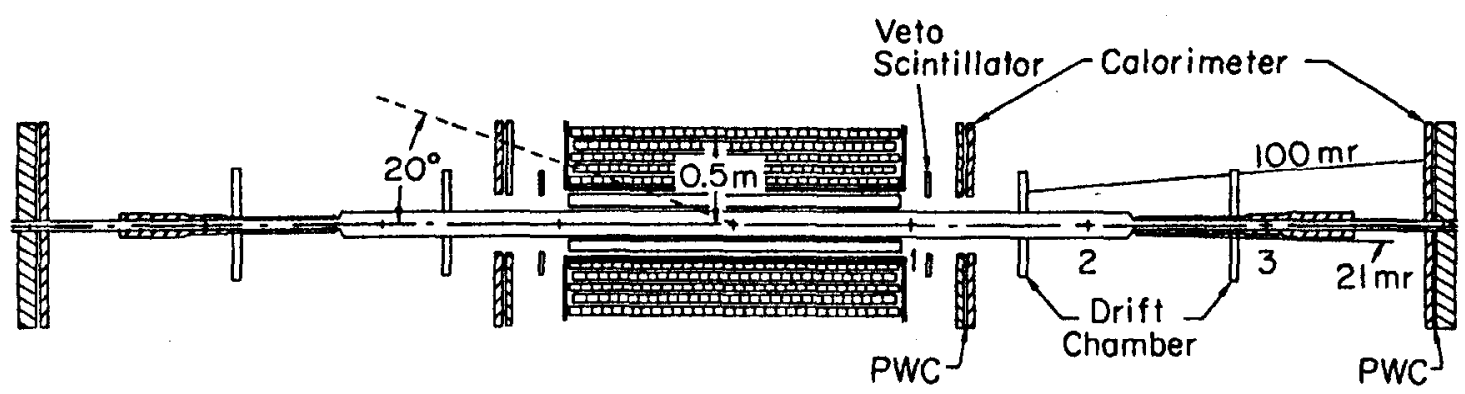

Figure 2.1. A vertical cross section of the ASP detector through the beam axis. The apparatus is $8.8 \mathrm{~m}$ long and $1.2 \mathrm{~m}$ wide.

The beampipe was a thin-walled vacuum chamber with a radius of $8 \mathrm{~cm}$. The central section of the beampipe covered the region $100 \mathrm{mr}<\theta<\pi-100 \mathrm{mr}$ and was made of .100 inch thick aluminium." The vacuum inside the beampipe was typically $\sim 10^{-9}$ Torr at the interaction point (IP). A tungsten mask below $21 \mathrm{mr}$ defined the forward detector acceptance. Between $50 \mathrm{mr}$ and $100 \mathrm{mr}$ the beampipe consisted of a 'window' of .090 inch aluminum, from 30 to $50 \mathrm{mr}$ there was a heavy stainless steel flange, and from 21 to $30 \mathrm{mr}$ the beampipe was .060 inch stainless steel.

A central tracker to detect charged particles, consisting of five planes of propor- 


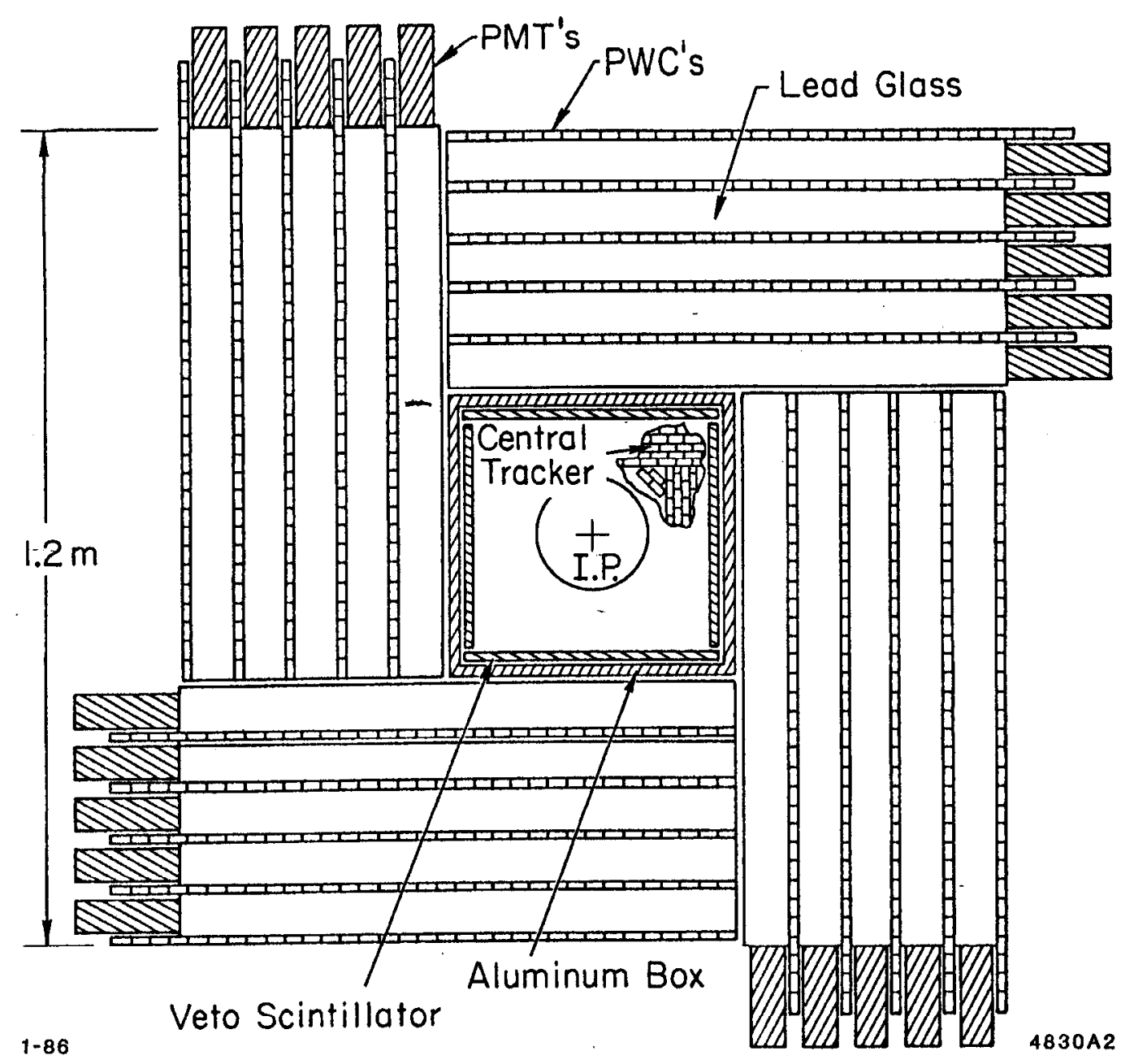

Figure 2.2. Cross section in the $x-y$ plane of the central calorimeter and tracking system. Only a section of the central tracker is shown; it completely surrounds the interaction point (IP).

tional chamber tubes, surrounded the beampipe. Each tube was $1.0 \times 2.3 \mathrm{~cm}^{2}$ in cross section and $2 \mathrm{~m}$ long. The walls were thinned by chemical etching to $0.3 \mathrm{~mm}$ to reduce the photon conversion rate. The tubes, which ran parallel to the beam, were staggered in the xy view so that charged particles from the beam axis could not pass completely undetected between them. Additional tubes were mounted in the corners to provide at least five layers of tracking at all values of $\phi$. The tubes were glued together and mounted on a Hexcell backplate. Each tube was strung with a 
sense wire of resistive Stablohm 800 wire, and both ends were read out to provide a z-coordinate by charge division. The central tracker was operated with a gas mixture of $48.3 \%$ argon, $48.3 \%$ ethane and $3.4 \%$ ethyl alcohol vapor. The alcohol vapor was added to prevent degradation of the wires due to high radiation exposure near the beampipe.

Surrounding the central tracker on all four sides was $2 \mathrm{~cm}$ of scintillator to provide redundancy in the identification of charged tracks. Each veto scintillator (VS) was read out on both ends with a piece of wavelength shifter viewed by a single photomultiplier tube (PMT). The signals from both ends were used to reconstruct a z-coordinate.

The lead-glass calorimeter consisted of 632 lead-glass bars, arranged in four quadrants of five layers each. Alternating layers had 31 or 32 bars, staggered to eliminate cracks and to provide optimal position resolution. Viewed along the beam direction, $\hat{\mathrm{z}}$, the pinwheel design left no radial gaps through which a charged particle or a photon from the interaction point could escape undetected. This arrangement of the lead-glass bars, with the long axis of each bar perpendicular to the beam direction, provided spatial information in the $\mathrm{xz}$ or $\mathrm{yz}$ planes.

The lead-glass bars measured $6 \times 6 \times 75 \mathrm{~cm}^{3}$ and were polished on both ends. The sides of the bars were smooth on an optical wavelength scale, but had ripples on the scale of a few $\mathrm{mm}$ which were created in the extrusion process. Extruded lead-glass bars are much cheaper to produce than polished lead-glass blocks, and it has been shown that their optical properties are equivalent. ${ }^{(23)}$

The type of lead glass used was Schott type F2; the composition was $11.8 \%$ lead, $29.7 \%$ oxygen, $21.5 \%$ silicon, $3.7 \%$ sodium, $3.3 \%$ potassium, and $.35 \%$ cerium by weight. The lead glass had a radiation length of $3.17 \mathrm{~cm}$ and an index of refraction of 1.58 . It was doped with $0.35 \%$ cerium to increase its radiation hardness ${ }^{(24)}$. The presence of cerium causes the lead glass to become slightly yellow, reducing its transmission at short wavelengths, but it protects against the dramatic transmission losses which occur with large doses of radiation. This effect was measured using an intense ${ }^{60}$ Co gamma ray source and one-inch cubes of lead-glass. For an integrated 
dose of $100 \mathrm{rad}$, undoped lead glass showed a $5 \%$ loss in transmission at $\lambda=500 \mathrm{~nm}$ (extrapolated to a length of $35 \mathrm{~cm}$.) while lead glass with $.3 \%$ cerium doping showed no loss after 100 rads. At greater exposures the transmission of undoped lead glass decreased rapidly while that of the cerium-doped lead glass decreased much more slowly.

Each lead-glass bar was read out on one end by an Amperex XP2212PC photomultiplier tube, a 12-stage PMT with high gain, good stability, and low noise. Prior to assembly, the PMTs were calibrated using a green Hewlett-Packard Superbright (HLMP-3950) light-emitting diode (LED). The PMT's were powered by a single LeCroy 1440 high voltage supply, which was controlled by the on-line VAX via CAMAC. This programmable supply was monitored every 4 minutes to verify the voltage settings and could automatically correct voltages which drifted. It proved to be a very reliable, stable power supply. The PMT calibration data were used to select groups of eight PMT's with roughly similar response to be powered from a single high voltage channel. This voltage was fanned out through a resistive divider which was then adjusted to provide the correct voltage for each tube.

The PMT's were glued to the bars using Stycast 6061 optical epoxy; each PMT covered $42 \%$ of the surface area of the end of a bar. The bars were wrapped with aluminum foil on five sides and a $\mu$-metal shield was put around each PMT to reduce the effect of external magnetic fields. The five lead-glass and PWC layers in each quadrant were then stacked in a light-tight box made of 0.75 inch thick aluminum. To minimize the non-active material inside the lead-glass calorimeter, the walls of the aluminum box were made half as thick where two quadrants abut. The calorimeter was supported internally by $6.8 \mathrm{~cm}$ high aluminum I-beams. Between adjacent layers of PWC six I-beams were used as spacers, creating 'shelves' on which the lead-glass bars were placed between thin layers of foam padding. This arrangement prevented undue stress on the lead-glass bars.

Each quadrant measured approximately $1.0 \times 0.5 \times 2.0 \mathrm{~m}^{3}$ and was an independent unit which was easily dismounted and transported. This was a necessary part of the design, because every component had to be carried in along the arcs from an adjacent 
interaction region. The two lower quadrants were mounted on rails and each was then bolted to the quadrant above, allowing the detector to be split by a remotelycontrolled hydraulic drive. This provided easy access to the beampipe and detector components whenever necessary. The ASP detector was located directly downstream from the PEP electron injection port, so to avoid excess radiation damage the two halves of the detector were moved away from the beamline whenever electrons were injected into the storage ring. To further protect the lead glass during injection, leadbrick walls were installet to shield it when it was in the open position. Electronic sensors ensured that the two halves were in the fully-closed position for data taking.

Interleaved with the lead glass were five planes of central proportional wire chambers (CPWC). The CPWC's were made from $2 \mathrm{~m}$ long aluminum extrusions with eight cells; four such extrusions formed one CPWC plane. Each of the 32 cells in a plane measured $1.2 \times 2.4 \mathrm{~cm}^{2}$ and was strung with one $48 \mu \mathrm{m}$ gold-plated tungsten sense wire. A mixture of $95 \%$ argon and $5 \%$ carbon dioxide gas flowed continuously through the CPWC's at atmospheric pressure. The CPWC wires were oriented with the sense wires parallel to the beam direction to provide spatial information in the $\mathrm{xy}$ plane, from which the azimuthal angle, $\phi$, could be reconstructed. Taken together, information from the CPWC's and lead-glass bars allowed for 3-dimensional track reconstruction.

Above the lead-glass calorimeter a time-of-flight (TOF) system consisting of 48 pieces of scintillator $3.45 \mathrm{~m}$ long, $20 \mathrm{~cm}$ wide and $2.5 \mathrm{~cm}$ thick was suspended from the ceiling. These counters were aligned with their long axes parallel to the beam direction in two overlapping groups which covered the lead-glass calorimeter in $+z$ and $-z$. Each scintillator was read out on both ends, allowing reconstruction of the $z$ coordinate. The TOF system was us̄ed primarily to reject cosmic ray events.

The lead-glass bars, CPWC's, central tracker and veto scintillators provided tracking and calorimetry for particles in the region $20^{\circ}<\theta<160^{\circ}$. A system of forward detectors completed the coverage to within $21 \mathrm{mr}$ of the beamline. Two calorimeters were located on each side of the central detector, at $z= \pm 1.5 \mathrm{~m}$ and 
$z= \pm 4.0 \mathrm{~m}$. The forward shower counters (FSC) (see fig. 2.3) were constructed from alternating sheets of lead $(0.6 \mathrm{~cm})$ and scintillator $(1.3 \mathrm{~cm})$ in modules which were 6 radiation lengths $\left(X_{0}\right)$ thick. The scintillator layers in each module were ganged together and read out by four wavelength shifter bars, each viewed by a single PMT. Two such modules were used for each of the inner FSC's, which overlapped the angular region covered by the central detector and extended the coverage to within $100 \mathrm{mr}$ of the beamline. Two crossed planes of PWC's, constructed of the same type of aluminum extrusion used the CPWC's, were inserted between the modules to measure the spatial position of electromagnetic showers. The outer FSC's, which covered the region between $21 \mathrm{mr}$ and $120 \mathrm{mr}$ from the beamline, consisted of three $6 X_{0}$ modulcs each. PWC's were again located at a depth of $6 X_{0}$, between the first and second modules. The additional material in the outer calorimeters reduced the probability that a photon would fail to convert in the detector, and provided good containment of showers from $e^{+} e^{-}$at low angles. The forward calorimeters provided a veto and calibration system for the ASP experiment as well as a luminosity monitor for the PEP storage ring.

Between the inner and outer FSC's on each side were drift chambers, located at $z= \pm 1.9 \mathrm{~m}$ and $z= \pm 3.0 \mathrm{~m}$. At each location there were two drift-chamber planes to measure both the $\mathrm{x}$ and $\mathrm{y}$ coordinates, so as to provide precise charged particle tracking between 21 and $100 \mathrm{mr}$. The drift chambers were operated with a gas mixture of $48.2 \%$ argon, $48.2 \%$ ethane and $1.6 \%$ ethyl alcohol.

\subsection{Online Calibration and Monitoring}

The entire detector was continuously monitored, with readings every four minutes; any voltage or current which strayed outside the prescribed boundaries would cause the data acquisition system to immediately shut down and would sound an alarm. A calibration was performed once during each eight-hour shift to provide upto-date constants and to detect any serious hardware malfunctions. In addition, a small fraction of the data was analyzed as it was being recorded to check for more subtle problems. At the end of each run the results of the analysis were printed out 


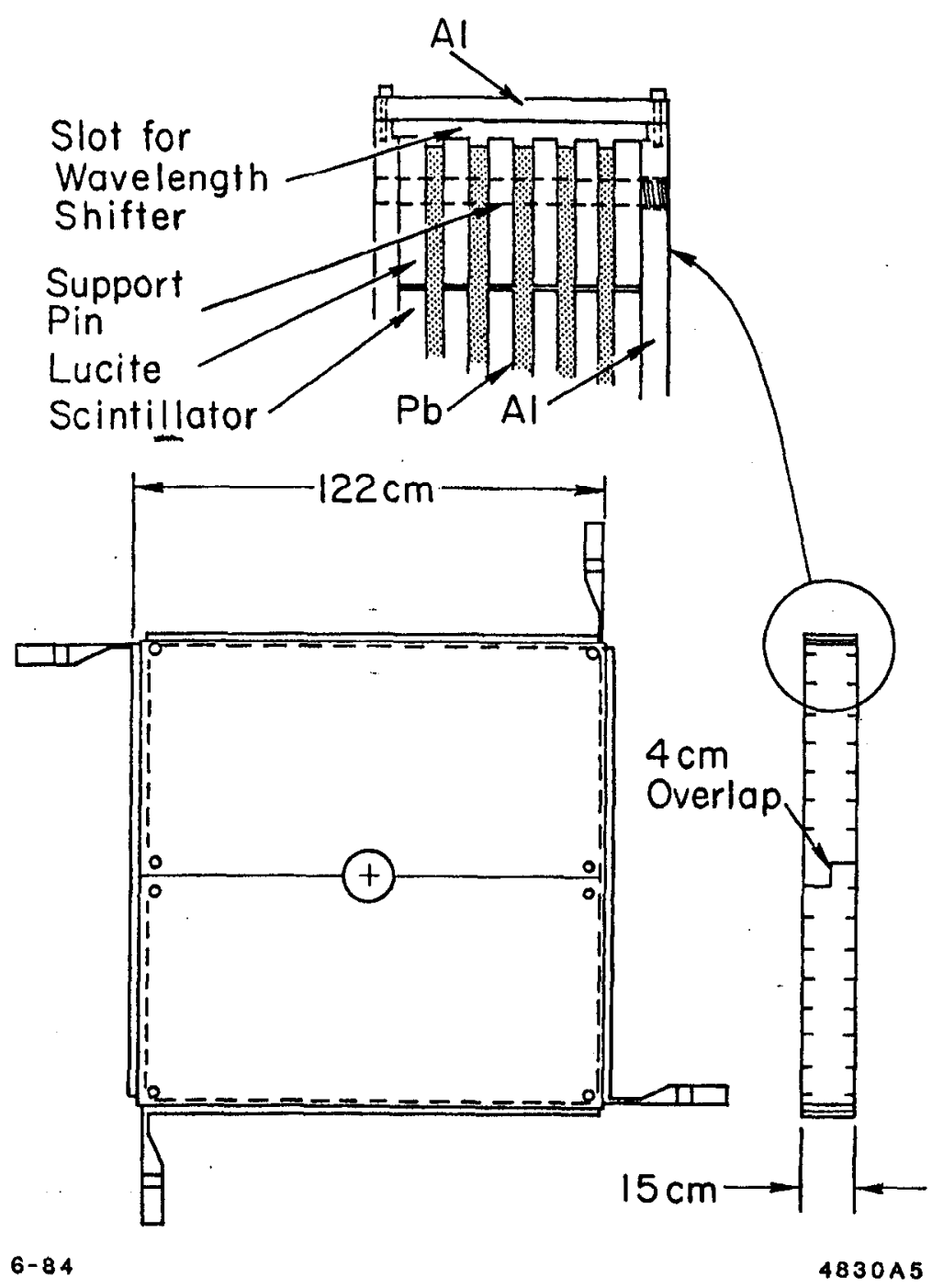

Figure 2.3. Front and side views of a forward shower counter module. The inset shows details of the construction of a corner of the module.

so the physicist on shift could check for any unsatisfactory results.

Stability of the lead-glass calorimeter response was very important. To this end the PMT's were monitored online with LED's of the same type used in the initial calibration. There was one LED per quadrant, each of which could be pulsed by a high-current pulser. $^{(25)}$ The light pulses were transmitted to each PMT with a light fiber. One end of each light fiber was embedded in the epoxy used to glue the PMT's to 
the lead-glass bars. The output of each LED was monitored by a reference PMT which also viewed a small $\mathrm{NaI}(\mathrm{Tl})$ scintillator crystal doped with ${ }^{241} \mathrm{Am}^{(26)}$ which served as a stable light source. ${ }^{(27)}$ This system was used to provide a relative calibration of the PMT's, but it was not used for an absolute calibration as the transmission of the light fiber connections drifted with time, and also the LED spectrum did not cover the full range of the PMT photocathode spectral sensitivity. The reliability of the phototubes was high: fewer than $1 \%$ of the tubes failed for any reason over the entire course of the experiment.

\subsection{The Trigger}

The signals from each of the 632 PMT's used to read out the lead-glass bars were first sent through a passive transformer splitter. One of the two resulting signals was sent to a SHAM-BADC system, ${ }^{(28)}$ a sample-and-hold module followed by an analogto-digital converter which also performed pedestal subtraction and gain correction. This provided the primary read-out of the calorimeter. The other half of the signals were summed, first in groups of eight from adjacent bars in the same layer, then with the other groups of eight in each layer to form 20 analog signals, one from each of the five layers in the four lead-glass quadrants.

The layer sums were then fanned out; see fig. 2.4. One copy was digitized and read out to provide a redundancy check against the SHAM-BADC system. It was also used in the offline code to correct for saturation in the BADC, which occurred when more than about $1.2 \mathrm{GeV}$ was deposited in a single bar. Another copy was summed to provide the total quadrant signals, which were summed in turn to form an analog signal proportional to the total calorimeter energy. These sums were also digitized and read out. A third copy of the layer sums was integrated and discriminated, as were the quadrant and total lead-glass sums, to provide digital inputs for the trigger logic. ${ }^{(29)}$

The ASP trigger decision was based on the discriminated signals from the layer, quadrant and total lead-glass sums, together with discriminated signals from the FSC's and veto scintillators. These digital inputs were sent to two Memory Logic 


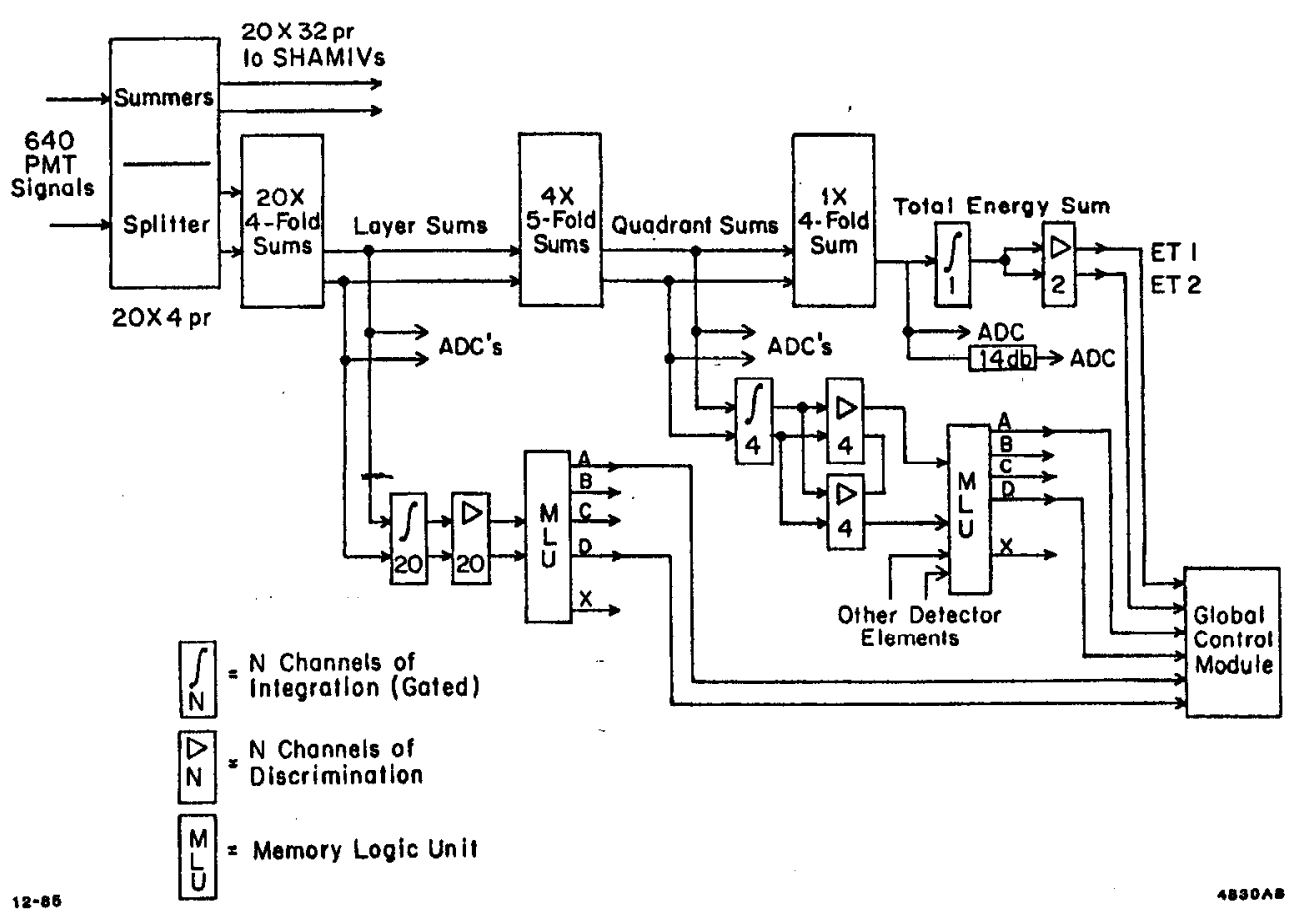

Figure 2.4. Summing circuitry for the lead glass signals.

Units (MLU's), which functioned as flexible look-up tables which could be programmed to recognize desired combinations of logical inputs from the various detector elements. The summary outputs from the MLU's were fed into the input latches of the Global Control module (GC), which would initiate digitization and event read-out by the host VAX $11 / 750$ computer. The trigger decision was made within $1 \mu \mathrm{s}$, less than the $2.4 \mu$ s between successive beam crossings in the PEP storage ring to avoid dead time. Several different triggers were formed from the layer, quadrant and total lead-glass sums. A summary is provided in table 2.1

The simplest trigger, $\mathrm{T} 1$, fired when the total signal from the lead-glass calorimeter exceeded a pre-determined threshold. This threshold, which was set at approximately $1.5 \mathrm{GeV}$, was determined by the requirement that it be greater than the coherent noise, which was insignificant on individual channels but became large when 
Table 2.1. Trigger Definitions.

\begin{tabular}{|l|c|}
\hline \multicolumn{1}{|c|}{ Trigger Name } & Description \\
\hline T1, 'Total Energy' & $>1.5 \mathrm{GeV}$ in lead-glass sum \\
\hline T2, 'One Quadrant' & $\begin{array}{c}>0.8 \mathrm{GeV} \text { in lead-glass quadrant with } \\
2 \text { layers }>0.1 \mathrm{GeV} \text {. Vetoed by S1. }\end{array}$ \\
\hline T3, 'Two Quadrants' & $\begin{array}{c}>0.4 \mathrm{GeV} \text { in each of } 2 \text { lead-glass quadrants } \\
+2 \text { layers }>0.1 \mathrm{GeV} \text {. Vetoed by S1. }\end{array}$ \\
\hline $\mathrm{T} 6$, 'Super-Trigger' & $\begin{array}{c}>0.7 \mathrm{GeV} \text { in lead-glass sum 'in-time', plus } \\
>0.15 \mathrm{GeV} \text { in back } 4 \text { layers of one quad } \\
\text { or in two opposite quads. Vetoed by } \mathrm{S} 1, \\
\text { or if }>2 \text { veto scintillators fired. }\end{array}$ \\
\hline T7, 'Radiative Bhabha' & $\begin{array}{c}>0.3 \mathrm{GeV} \text { in lead-glass sum plus } \\
>7 \mathrm{GeV} \text { in opposite side FSC's. }\end{array}$ \\
\hline T8, 'Farly VS Cosmic' & \begin{tabular}{c}
1 or more VS, $>5$ ns before beam crossing. \\
\hline T9, 'Random'
\end{tabular} Random triggers from free-running oscillator. \\
\hline T10, 'Bhabha' & $>7 \mathrm{GeV}$ in opposite side FSC's \\
\hline S1 Veto & Sum of outer FSC's $>1$ GeV and $<7 \mathrm{GeV}$. \\
\hline
\end{tabular}

summed over all 632 channels.

Two other triggers, T2 and T3, were specifically designed for single photon events. For the case in which the photon shower was contained in one quadrant, the $\mathrm{T} 2$ trigger required at least $0.8 \mathrm{GeV}$ in one quadrant with at least two layers over $0.1 \mathrm{GeV}$. The layer requirement eliminated many events from beam-gas collisions, which can produce a $\pi^{\circ}$ at low angles which would just hit the inner layers. The T3 trigger required at least $0.4 \mathrm{GeV}$ in each of two quadrants, with the additional requirement of at least two layers in one quadrant or at least one layer in each of two adjacent quadrants. The $\mathrm{T} 2$ and $\mathrm{T} 3$ triggers were vetoed if there was more than 1 $\mathrm{GeV}$ and less than $7 \mathrm{GeV}$ deposited in the outer FSC modules, again to eliminate beam-gas events.

The lowest threshold was achieved for the $\mathrm{T} 6$ trigger, which required $0.7 \mathrm{GeV}$ in 
the total lead-glass sum. In addition, at least $0.15 \mathrm{GeV}$ was required in the back four layers of one and only one quadrant, or in two opposite quadrants. No more than two of the four veto scintillators surrounding the central tracker were allowed to be above threshold, and the lead-glass energy was required to be 'in-time', within \pm 20 ns of the beam crossing. Finally, the T6 trigger was vetoed by the same FSC veto applied to the T2 and T3 triggers. The combined efficiency of the 'lead-glass triggers', T1,T2,T3 and T6, is shown in fig. 2.5 as a function of total-lead glass energy, measured using kinematically-fit radiative-Bhabhas.

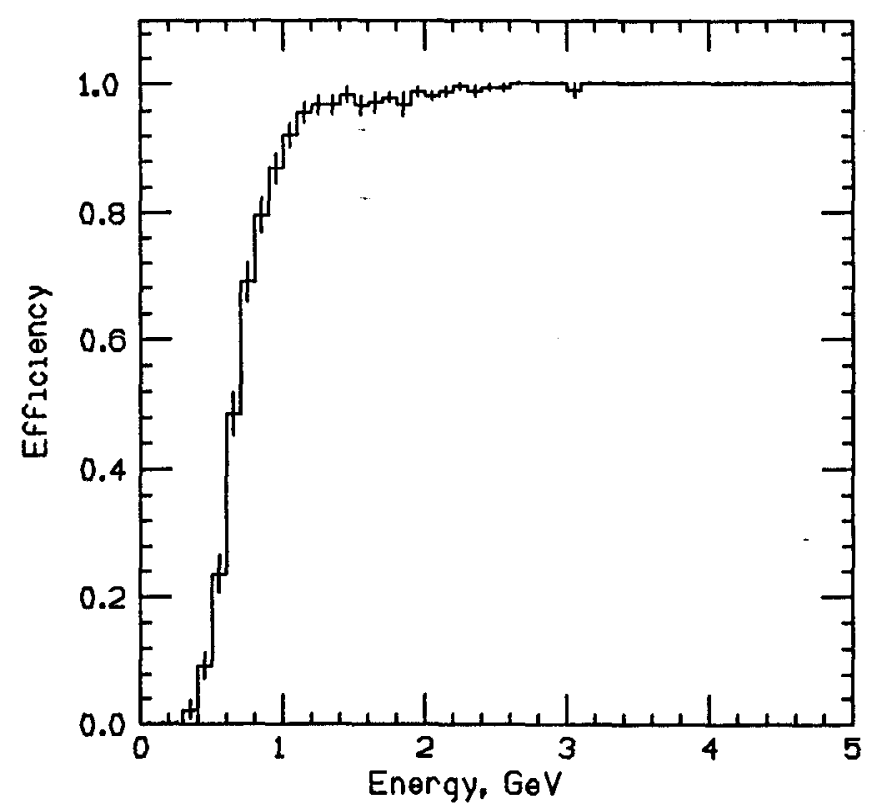

Figure 2.5. Efficiency of lead-glass triggers as a function of total lead-glass energy.

The T7 trigger was designed to log out radiative Bhabha events with two forward tracks and one track in the glass. This trigger required at least $0.3 \mathrm{GeV}$ in the lead glass plus a coincidence between two FSC's with at least $7 \mathrm{GeV}$ on either side of the detector. Because the T7 trigger threshold was significantly lower than the other lead-glass trigger thresholds, it was useful for studying the performance of the other triggers. In addition, kinematically-fitted radiative Bhabhas provided a means of understanding many aspects of the detector performance.

Other diagnostic triggers included the early VS cosmic trigger, which fired when a charged particle was detected in the veto scintillators surrounding the central tracker 
in a $15 \mathrm{~ns}$ time window which ended 5 ns before the beam crossing. Low-angle Bhabhas in which more than $7 \mathrm{GeV}$ was deposited in both inner or both outer FSC modules were recorded for luminosity studies. (Because of the high event rate for this process a pre-scale factor of 600 for the outer and 20 for the inner FSC's was imposed.) Random beam crossings were also recorded to study detector occupancy. The total trigger rate of the ASP detector was typically $4.5 \mathrm{~Hz}$, and the live time was typically about $90 \%$. 


\section{Event Reconstruction}

The raw data consists of signals from each detector element. These signals must be calibrated to correct for channel-to-channel variations and to determine the absolute scale. Then pattern recognition, or 'tracking', must be performed on all of the signals to determine the particle trajectories in an event. Once the events have been tracked, the event selection can be made. In this chapter the calibration and tracking procedures for the lead-glass calorimeter are described.

\subsection{Off-line Calibration}

Cosmic ray muons recorded by the early VS cosmic trigger were used to perform an off-line calibration of the PMT response. This sample was tracked and the signal deposited in each bar was corrected for path length. The average path-corrected signal displayed a strong dependence on both the distance of the track from the PMT and on the angle of the track with respect to the long axis of the bar (see fig. 3.1). The dependence on distance was due to reflection losses and attenuation of the signal in the lead glass, modified by geometry- and frequency-dependent increases in light collection when the distance became less than a few $\mathrm{cm}$. The dependence on angle was due to the fact that the Cerenkov light gencrated by a particle traveling towards the PMT travels a shorter distance in the lead glass than light generated by particles heading away from the PMT, which must travel to the far end and be reflected back. This effect can be seen by comparing fig. 3.1b with fig 3.1a. A change in direction 
also changes the angle of incidence of the light as it travels down the bar by internal reflection and hence changes the total distance travelled in the glass.

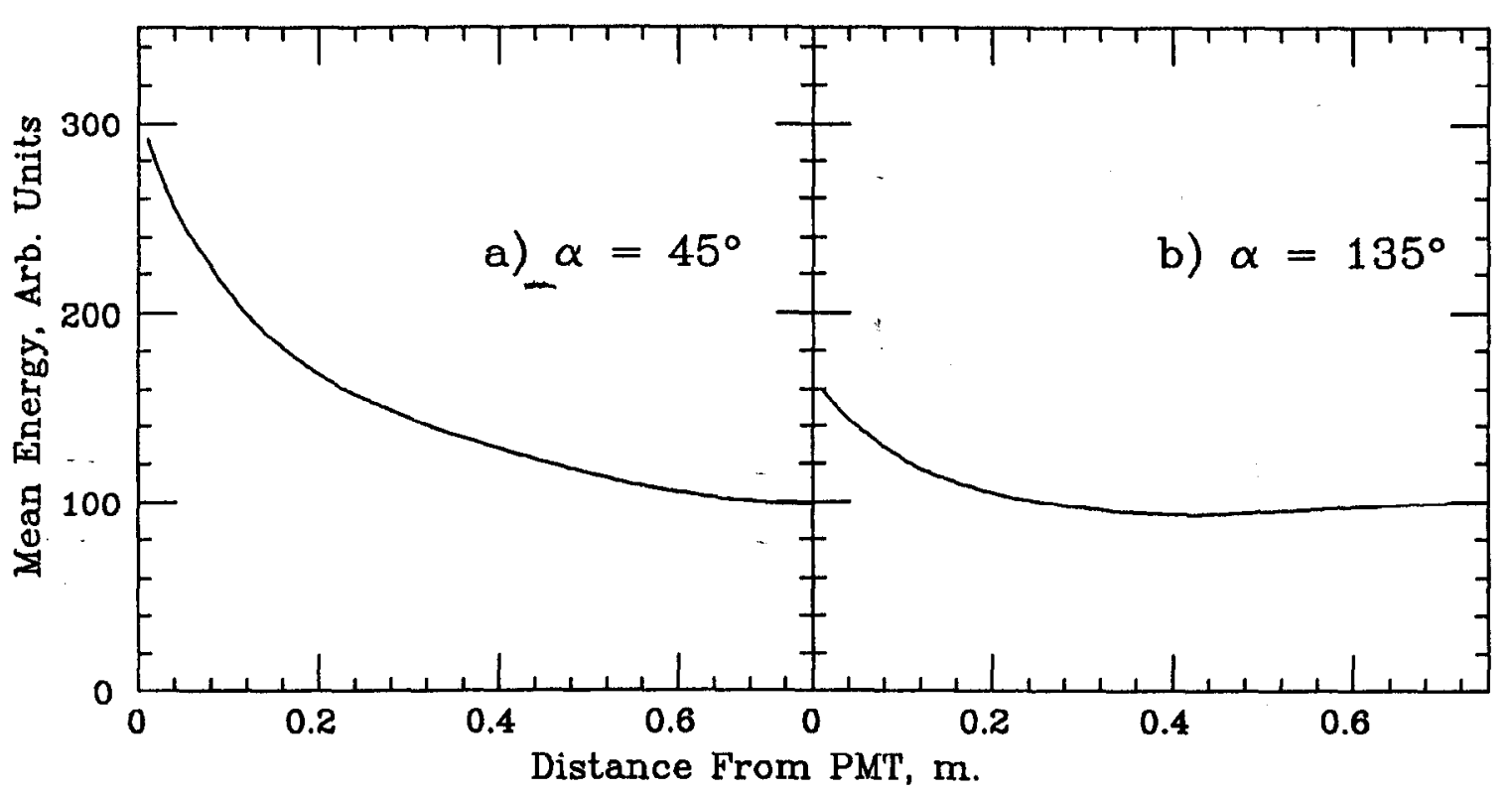

Figure 3.1. Attenuation in the lead glass bar as a function of distance and angle. In a), the track is pointing towards the PMT at an angle of $45^{\circ}$, and in b) it is pointing away.

Each lead-glass quadrant was oriented in a different direction, with some PMT's pointing up, some down and others oriented horizontally. Therefore the angular distribution of the predominantly down-going cosmic rays with respect to the axis of the lead-glass bars was different in each quadrant. This resulted in a different average path-length corrected signal in each quadrant. To compensate for attenuation effects, a correction was applied on an event-by-event basis. The correction factor was a function of both the distance of the track from the PMT and the angle of the track with respect to the bar, taken from a look-up table compiled from plots such as those in fig. 3.1. The peak was extracted from the resulting spectrum for each PMT and this value was used to normalize its response. Most PMT's had multiplicative normalization factors in the range from .5 to 1.5 , with a handful extending up to 2 .

This cosmic-ray calibration was more accurate than the one which had been 
performed with an LED prior to installation because the spectral response of the PMT's was integrated over the correct frequency range of Čerenkov light. In addition, any channel-to-channel variation in the clectronics used to read out the PMT's was automatically included in the calibration. This calibration was also used to check for evidence of radiation damage, which would cause a time dependent decrease in the transmission of the lead-glass bars. No significant decrease in response was observed. Moving the detector away from the beampipe during injection and the installation of lead-brick walls were responsible for a significant reduction in the integrated radiation dose seen by the lead glass, and the addition of cerium to the lead glass prevented any significant damage from the radiation which was absorbed.

\subsection{Energy Determination}

The Čerenkov light collected from an electromagnetic shower in lead glass is proportional to the integrated path length traveled by all charged particles in the shower which are above the Čerenkov threshold ( $0.7 \mathrm{MeV}$ for electrons), and is therefore proportional to the energy of the shower. The light is transmitted by internal reflection down the bar to the PMT. After correcting for the effects of differing PMT gains using the constants derived in the off-line calibration, the signals must be corrected for attenuation. The event was tracked first, and then the same look-up table used in the off-line calibration was applied, using the track parameters to calculate the angle and distance of the shower from the PMT.

Further corrections were made for leakage, pre-radiation and the absorption of energy in the non-active material inside the calorimeter. The amount of energy which leaks out of the calorimeter is a function of the total thickness (in radiation lengths) and the incident particle energy. Using the EGS Monte Carlo ${ }^{(30)}$ to simulate electromagnetic cascades, the following empirical approximation was derived to correct for leakage on the mean:

$$
E_{l e a k}=2.0 \times e^{-.3 X} \times E_{\text {inc }}{ }^{1.4} \mathrm{GeV}
$$

where $E_{\text {leak }}$ is the energy lost in $\mathrm{GeV}, \mathrm{X}$ is the thickness of the detector in radiation 
lengths along the trajectory of the track, and $E_{i n c}$ is the incident energy in GeV. The thickness of the detector at $\phi=90^{\circ}$ varied from $10 X_{0}$ at $\theta=90^{\circ}$ to $20 X_{0}$ at $\theta=30^{\circ}$. The thinnest part of the detector was in the corners between quadrants, where $X \simeq 8$ $X_{0}$ at $\theta=90^{\circ}$; see fig. 3.2. For photons, the estimated point of conversion in the lead glass was also used in the calculation of X. A first estimate of $E_{l c a k}$ was obtained by substituting the observed energy for $E_{i n c}$, then $E_{o b s}+E_{l e a k}$ was substituted for $E_{i n c}$ and this procedure was iterated until the estimate of $E_{l e a k}$ converged to within $5 \%$ of the previous estimate for $E_{l e \overrightarrow{a k}}$. For a $1 \mathrm{GeV}$ particle at normal incidence, the mean energy lost is about $100 \mathrm{MeV}$, or $10 \%$, and at $\theta=30^{\circ}$ this is reduced to $0.5 \%$. The corresponding numbers for a particle of $14.5 \mathrm{GeV}$ are $30 \%$ and $1.5 \%$. This correction is clearly most important at wide angles. No attempt was made to take into account fluctuations in leakage.

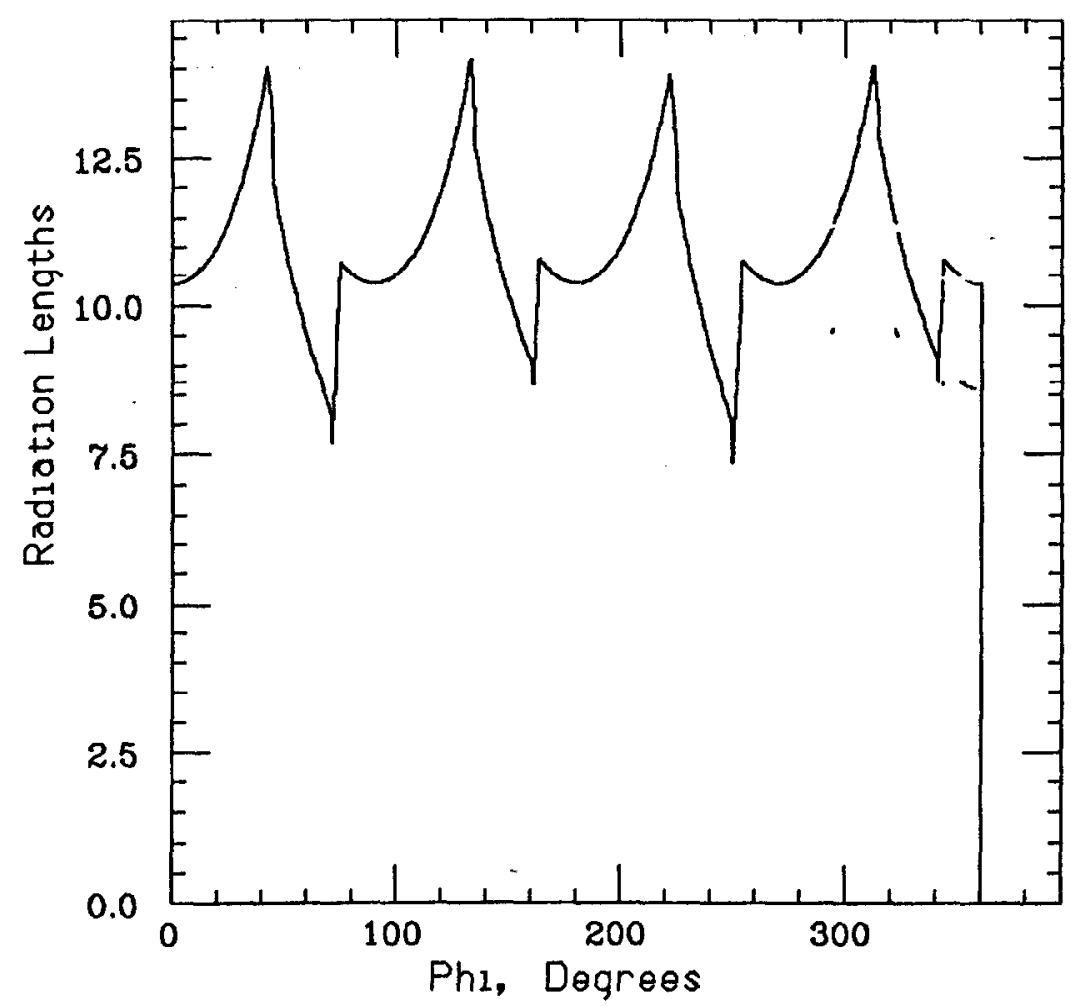

Figure 3.2. Thickness of the lead-glass calorimeter in radiation lengths vs. $\phi$, at $\theta=90^{\circ}$.

Radiation of charged particles in the material preceding the calorimeter and absorption of energy in the non-active material have also been studied using EGS. 
At normal incidence a particle will traverse $0.36 X_{0}$ before entering the lead-glass calorimeter. This is primarily due to the 0.75 inch thick aluminum box enclosing each quadrant. A $1 \mathrm{GeV}$ charged particle deposits on average $20 \mathrm{MeV}$ in this material. The pre-radiation increases exponentially with radiation length and there is also a weak dependence on the energy of the incident particle. To correct for this loss, the following empirical formula was developed using EGS simulation:

$$
E_{p r e}=.008 \times e^{2.6 X} \times e^{.04 E_{i n c}} \mathrm{GeV}
$$

In this formula, $E_{p r e}$ is the amount energy deposited in $\mathrm{GeV}, \mathrm{X}$ is the amount of material in radiation lengths traversed before entering the lead glass, and $E_{\text {inc }}$ is again the incident particle energy in $\mathrm{GeV}$.

Energy is also absorbed in the Al walls of the PWC's, the I-beams, and most importantly, the 0.75 inch of aluminum between quadrants. The correction for this energy loss is a strong function of $\phi$, and becomes largest when a track is heading directly between two lead-glass quadrants. Using EGS, the $\phi$ dependence was mapped out and a look-up table was constructed. The fraction of energy absorbed relative to the total incident energy is roughly constant with energy, and varies from $4.5 \%$ at normal incidence to $12 \%$ at $\phi=60^{\circ}$, which is approximately where the boundary between quadrants is located.

After all these corrections had been applied, the energy resolution was measured using a sample of approximately $8 \times 10^{4}$ kinematically fitted $e^{+} e^{-} \gamma$ events. Events were selected in which two of the outgoing particles were between 21 and $180 \mathrm{mr}$ from the beamline, where the long lever arm of the forward systems afforded a precise measurement of thcir position. The third particle, either an electron or photon, was required to be in the lead-glass calorimeter. (The energy of the track in the lead glass was kinematically limited to less than about $4 \mathrm{GeV}$ for this class of events.) Using the known center of mass energy $(\sqrt{s}=29 \mathrm{GeV}$ at PEP) and the measured angles of all three tracks, the absolute energy scale of the lead-glass and the forward calorimeters was determined in a one-constraint fit using the SQUAW fitting routine. 
(31) Once the overall normalization had been established, the calorimeter resolution was determined by plotting the difference between the measured energy and the fitted energy. The results for the lead glass are displayed as a function of energy in fig. 3.3. The curve is well described by the resolution function

$$
\frac{\sigma_{E}}{E}=\frac{10.0 \%}{\sqrt{E}}
$$

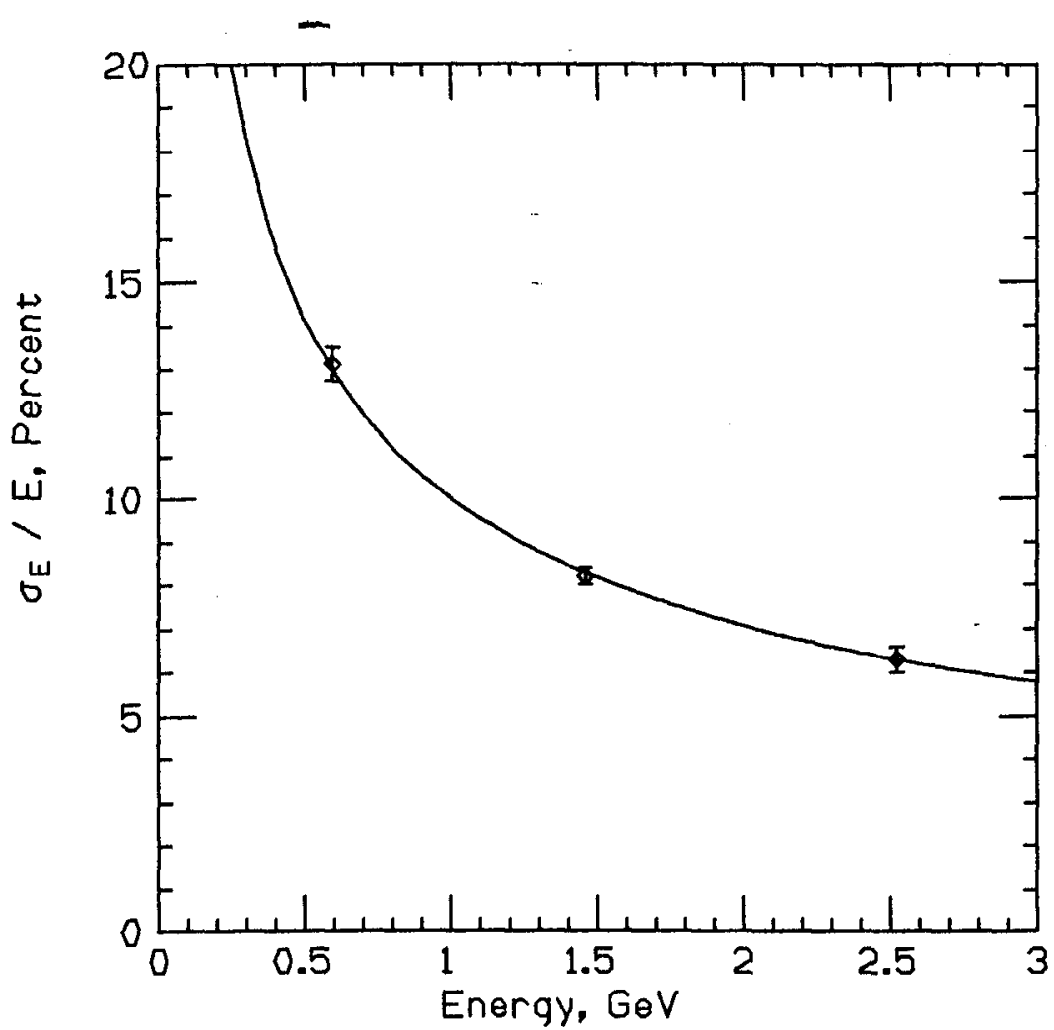

Figure 3.3. Resolution in the lead glass bar as a function of energy.

\subsection{Tracking}

The tracking proceeded in several stages. The boundaries of each shower were first determined with a cluster-finding algorithm. Fluctuations in the development of an electromagnetic shower create gaps in the pattern of energy deposition, while on the other hand two showers which are close together in the plane of a quadrant may overlap. The clustering algorithm therefore allowed gaps, but was also sensitive 
to peaks and valleys and would start a new cluster when large enough fluctuations in signal were encountered. Clusters were not continued across quadrant boundaries; clusters from a single shower which extended into adjacent quadrants were combined at a later stage of the tracking.

A two-dimensional total least squares fit was then performed for each cluster. This fit found the axis about which the sum of the squares of the perpendicular distances to each bar, $D_{i}$, was minimized. The signal in each bar corrected for PMT gain, $S_{i}$, was used to weight each term. (The attenuation correction could not be applied at this stage because the angles of the track were not yet determined.) The quantity to be minimized was:

$$
M_{W^{2}}=\sum_{i} S_{i} D_{i}^{2}
$$

A slope and intercept in the plane of the quadrant ( $\mathrm{xz}$ or $\mathrm{yz}$ ) were extracted from this fit.

The signals observed in the central PWC's, the central tracker, and the forward systems were all tracked independently and combined with the lead-glass clusters to reconstruct an event topology. To accomplish this, the track segments from each system were reduced to vector and error matrices. A topology finding routine then identified the segments from each system which could belong together in a track. A least-squares fit was performed, and if the $\chi^{2}$ was satisfactory a track was created utilizing all the information from the various systems. If the $\chi^{2}$ was unsatisfactory, the segment which contributed the most to the $\chi^{2}$ was dropped and the fit was tried again. Clusters from a shower which crossed the boundary between two quadrants wcre combined in a similar fashion. The clusters were required to match based on a $\chi^{2}$ formed from their $\theta$ values and errors, and information from the other central tracking systems was used in the fit. Tracks from minimum-ionizing particles were treated in the same way as electromagnetic showers; the tracking code worked equally well for both.

Ambiguities arose in matching lead-glass clusters and PWC clusters when there 
were multiple tracks per quadrant. For charged tracks ihis could usually be resolved by the central tracker. However if there were multiple neutral tracks in a quadrant, the tracking algorithm had to rely on a comparison of the signal deposited in each layer of the lead glass and PWC clusters. From this comparison the best match between the lead-glass and central PWC systems was determined, based on how well the patterns of energy deposition in the two systems matched. When two showers had very different patterns this method worked well; however if they were similar the likelihood of choosing the wrong combination increased. Because of this limitation the tracking worked best for low multiplicity events.

Bhabha events were used to study the angular resolution of the lead glass. The quantity which is actually measured with the lead-glass calorimeter is $\theta_{p}$, the polar angle projected into the $\mathrm{xz}$ or yz plane. The resolution in $\theta_{p}$ improves at lower angles because the lever arm is greater as the distance to the interaction point increases. The resolution averaged over all angles is $\sigma=1.9^{\circ}$. The angular resolution of the lead glass was also a function of energy, becoming worse at lower energies because the position of the shower centroid was less well measured. For showers from kinematically fitted radiative Bhabha events, ranging in energy from 0.5 to $3 \mathrm{GeV}$, the average resolution was $\sigma=4.4^{\circ}$.

The spatial origin of a shower in the lead glass was characterized by the distance of closest approach to the beamspot in the $\mathrm{xz}$ or yz plane, $R_{z}$. This quantity was used because the resolution is then approximately independent of $\theta_{p}$. For $14.5 \mathrm{GeV}$ showers from Bhabha events the $R_{z}$ distribution was Gaussian, with an average resolution of $\sigma=2.6 \mathrm{~cm}$. The resolution is shown as a function of $\theta_{p}$ in fig. 3.4. For low-energy showers, such as those from radiative Bhabha events, the distribution was Gaussian with $\sigma=2.8 \mathrm{~cm}$ and a small exponential tail.

Timing information from the lead glass was obtained for each quadrant, and corrections were applied using the tracking information. Both the path length of the track from the interaction point to the lead-glass calorimeter and the distance the light traveled down the bar to the PMT were taken into account. After these corrections were applied the timing resolution was $\sigma=1.2 \mathrm{~ns}$ for a $1 \mathrm{GeV}$ shower and 


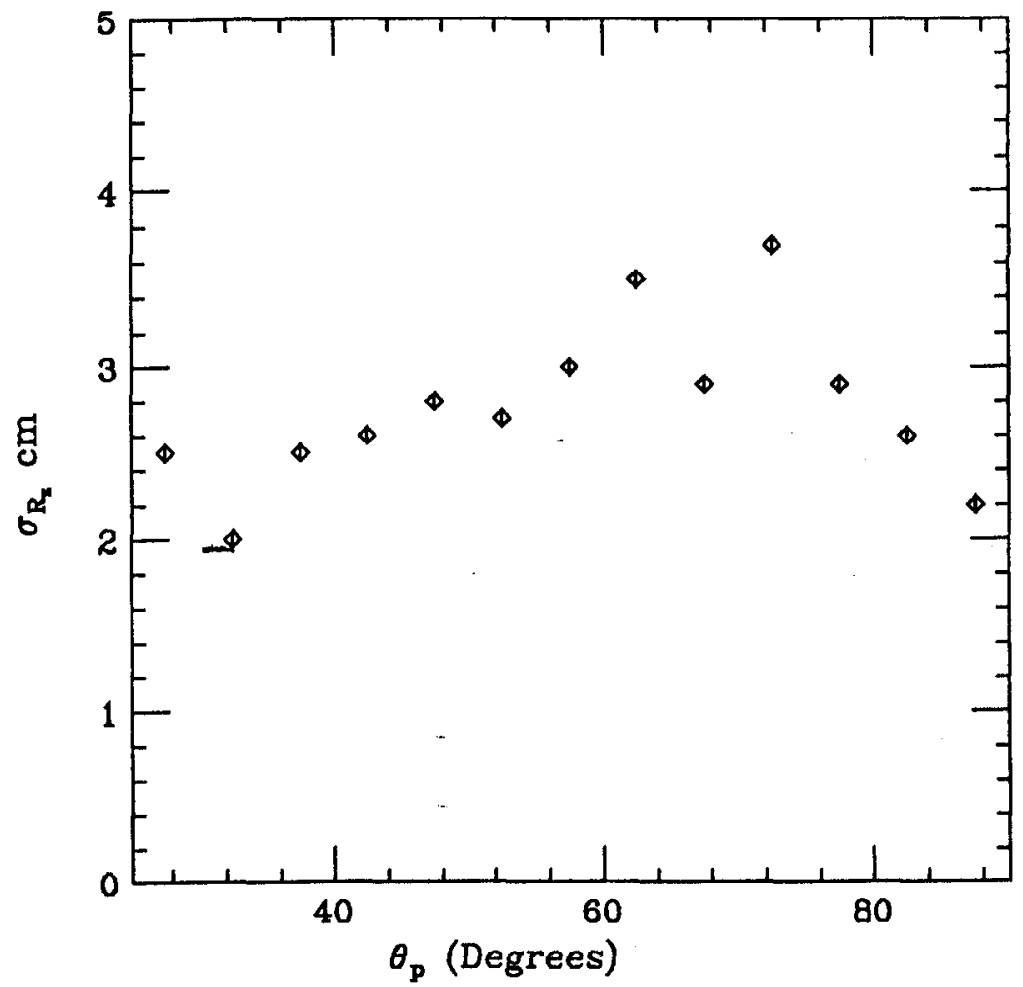

Figure 3.4. Resolution in the lead glass bar for the beam intercept as a function of angle. $\sigma=1.0$ ns above $2.5 \mathrm{GcV}$ 


\title{
Event Simulation
}

\begin{abstract}
- About $5 \%$ of all $\eta$ 's and $15 \%$ of all $\eta^{\prime}$ 's produced in two-photon interactions which subsequently decayed into two photons were included in the final data sample. Some did not decay into the sensitive parts of the detector, and others failed to fire the trigger. Some events were removed by cuts designed to reduce backgrounds from other processcs. Understanding the detector acceptance and event selection efficiency is a very important aspect of the analysis. To achieve this with a high degree of accuracy, Monte Carlo methods and a detailed model of detector response are employed to perform the calculations.

The first step is to generate $\eta$ and $\eta^{\prime}$ events according to the two-photon production cross section. This procedure is described in section 4.1. Next the generated photons are passed through the detector simulation routines, described in section 4.2. A comparison of the simulation with real data is also presented in this section, to show how well the detector response is reproduced. The trigger logic is then applied to the raw data, as described in section 4.3 , to determine whether the generated event would have fired the trigger and been logged out.

The simulated events can be tracked and processed using the same event selection routines which are used to filter the data. This is the subject of the next chapter.
\end{abstract}




\subsection{Event Generation}

The differential cross section for two-photon production of $\eta$ or $\eta^{\prime}$ mesons, eq. 1.14 , has been formulated by Vermaseren ${ }^{(32)}$ in a form which is convenient for numerical integration. A Monte Carlo based on this formulation has been used which generates unweighted events and simultaneously calculates the total cross section, assuming that $\Gamma_{\gamma \gamma}=1 \mathrm{keV}$. The cross sections are therefore calculated in $\mathrm{nb} / \mathrm{keV}$, so that $\Gamma_{\gamma \gamma}$ is given by the ratio of the experimentally obtained value for the cross section to the calculated cross section. The processes $e^{+} e^{-} \rightarrow\left(e^{+} e^{-}\right) \eta$ and $e^{+} e^{-} \rightarrow\left(e^{+} e^{-}\right) \eta^{\prime}$ were generated with $M_{\eta}=548.8 \mathrm{MeV}$ and $M_{\eta^{\prime}}=957.6 \mathrm{MeV}$; all of the generated events were made to decay into two photons, the final state of interest for this analysis.

The $Q^{2}$ dependence of the cross section for two-photon production of the $\eta$ and $\eta^{\prime}$ mesons is illustrated in table 4.1 , where the calculated cross sections are given with no form factor and also with the form factor of Brodsky and Lepage, ${ }^{(33)}$

$$
F\left(q_{1}^{2}, q_{2}^{2}\right)=\frac{1}{\left(1+\frac{q_{1}^{2}}{M^{2}}\right)\left(1+\frac{q_{2}^{2}}{M^{2}}\right)}, \quad M^{2} \sim .68 \mathrm{GeV}^{2}
$$

This form factor differs from the $\rho$ form factor only in the value of the mass, which for the $\rho$ form factor is $M^{2}=M_{\rho}^{2}=.59 \mathrm{GeV}^{2}$. The total production cross section is significantly suppressed by the form factor, but for events within the ASP detector acceptance (defined as $20^{\circ}<\theta_{\mathrm{p}}<160^{\circ}$ for both photons, and both electrons within $21 \mathrm{mr}$ of the beamline) the difference in the cross section with and without the form factor is slight, a $1.5 \%$ reduction for $\eta$ events and $1.8 \%$ for $\eta^{\prime}$ events. This is because the requirement that the scattering angle of the electrons be less than $21 \mathrm{mr}$ limits the maximum $Q^{2}$ to less than $.093 \mathrm{GeV}^{2}$.

Events were generated about the measured beam positions with a gaussian distribution characterized by $\sigma=0.5 \mathrm{~cm}$ in $\hat{x}$ and $\hat{y}$ and $\sigma=1.5 \mathrm{~cm}$ in $\hat{z}$. The position of the beamspot was measured using the forward drift chambers; it varied with time in $\hat{x}$ and $\hat{y}$ but remained the same in $\hat{z}$. Run numbers from both data cycles were chosen in the event generation process, using luminosity weighting. This ensured that run-dependent constants, such as the beam spot position, the PMT gain factors and 
Table 4.1. $Q^{2}$ Dependence of Two-Photon Cross Sections

\begin{tabular}{|c|c|c|}
\hline & $\sigma\left(e^{+} e^{-} \rightarrow e^{+} c^{-} \eta\right), \mathrm{nb} / \mathrm{kcV}$ & $\sigma\left(e^{+} e^{-} \rightarrow c^{+} e^{-} \eta^{\prime}\right), \mathrm{nb} / \mathrm{keV}$ \\
\hline$\sigma_{t o t}$, no form factor & $3.185 \pm .001$ & $0.5219 \pm .0002$ \\
\hline$\sigma_{a c c}$, no form factor & $0.686 \pm .003$ & $0.1100 \pm .0005$ \\
\hline$\sigma_{\text {tot }}$, B.L. form factor & $2.560 \pm .001$ & $0.3620 \pm .0002$ \\
\hline$\sigma_{a c c}$, B.L. form factor & $0.676 \pm .002$ & $0.1080 \pm .0004$ \\
\hline
\end{tabular}

the list of dead PMT's, were properly sampled.

\subsection{Detector Simulation}

The generated events, consisting of four-vectors for each produced particle, were passed through the detector simulation package, which is based on the EGS4 Monte Carlo code ${ }^{(30)}$ EGS provides a detailed, step-by-step simulation of the electromagnetic cascade process. Because we are concerned only with final states consisting of electrons or photons, EGS can be used to simulate all final states of interest.

The simulation proceeds in the following manner: the initial state particle is stepped through the detector by EGS, which has a detailed map of the detector geometry including all components of the central detector. Using a random number generator and the cross sections for all important processes, EGS determines when the first interaction occurs. For example, a photon will produce an electron-positron pair in the field of a nucleus. Then the electron and positron are stepped through the detector, and each time a photon is produced by bremsstrahlung it is added to the list and stepped through until it pair produces, and so on. In this way an electromagnetic cascade is generated.

Each time one of the particles in the shower takes a step, a routine called AUSGAB is called from EGS. In $\triangle$ USGAB, the measurable effects produced by each particle are accumulated. If the particle is in the beampipe, or a support bracket, or some other insensitive part of the apparatus, nothing is registered, but when a charged particle enters a sensitive part of the apparatus, the appropriate detector 
response is simulated.

The simulation of electromagnetic showers using EGS is very CPU intensive, and the computer time required grows linearly with energy. To reduce the time required somewhat, therc is an energy cutoff in EGS below which a particle is not transported any further. This cutoff is adjustable, and has been set to $1.5 \mathrm{MeV}$ for electrons and $0.5 \mathrm{MeV}$ for photons. The detector response to the remaining energy deposit is estimated and accumulated in AUSGAB when the particle is discarded.

The passage of a charged particle through lead glass results in the emission of Cerenkov light. The amount of Čerenkov light deposited by each charged particle in the lead glass is proportional to the step length $\times\left(1-\frac{\beta_{c}^{2}}{\beta^{2}}\right)$, where $\beta=\frac{v}{c}$ is the particle velocity divided by the speed of light and $\beta_{c}=\frac{1}{n}$ is the threshold for emission of Čcrcnkov light in a medium of refractive index $n$. (In the lead glass used in this experiment, $n=1.58$ and $\beta_{c}=.63$.) The light is emitted in a cone around the trajectory of the particle with an opening angle given by $\theta_{c}=\arccos \left(\frac{1}{\beta n}\right)$. For a particle traveling close to the speed of light in lead glass $\theta_{c} \sim 50^{\circ}$. The Cerenkov light travels down the lead-glass bar by internal reflection, eventually reaching the photo-cathode of the PMT which is glued to one end.

In order to simulate this process exactly, one would need a very sophisticated algorithm to trace the light rays from the Čerenkov cone as they reflect down the length of the lead-glass bar, determining the loss by attenuation. Instead, we have used a look-up table which gives the attenuation factor as a function of the distance of the particle from the PMT and the angle of the primary particle which initiated the shower. This look-up table was compiled using high-energy muons from cosmic-ray data. It is the inverse of the look-up table used to correct the raw data for attenuation after the tracking has been performed, as described in the previous chapter. After accumulating all the Cerenkov signals in an event, the gain factor for each PMT is applicd to the data. This factor is set to zero for any dead PMT's.

Attenuation and PMT gain must be included in the simulated data for two reasons. First, the trigger decision is based on raw data, so these effects are important in correctly estimating trigger efficiency. Secondly, it is important to make the simu- 
lated data as close as possible to real data in order that the exact same routines may be used to process both. Then if there are any bugs in any of the routines, they will bc included in the efficiency calculation.

As a check of the simulation of raw data using EGS, a comparison was made with real data. Radiative Bhabha events with one electron or photon in the lead glass and two forward tracks were kinematically fitted, as described in Chapter 3. The energy of the particle in the lead glass was extracted from the fit, and an electron or photon in the same location and with the fitted energy was simulated. This procedure was repeated for many events over the entire detector acceptance. The conversion factor from EGS unils lo raw data units was determined by requiring the mean value of the ratio of simulated data to real data to be equal to 1 . The ratio of the average simulated lead-glass signal to the average data signal is plotted as a function of azimuthal angle in fig. 4.1. Good agreement is found, indicating that the attenuation of Cerenkov light is reasonably well simulated. (For comparison, fig. 3.1 showed that attenuation produced changes in the observed lead-glass signal of up to a factor of 3 .)

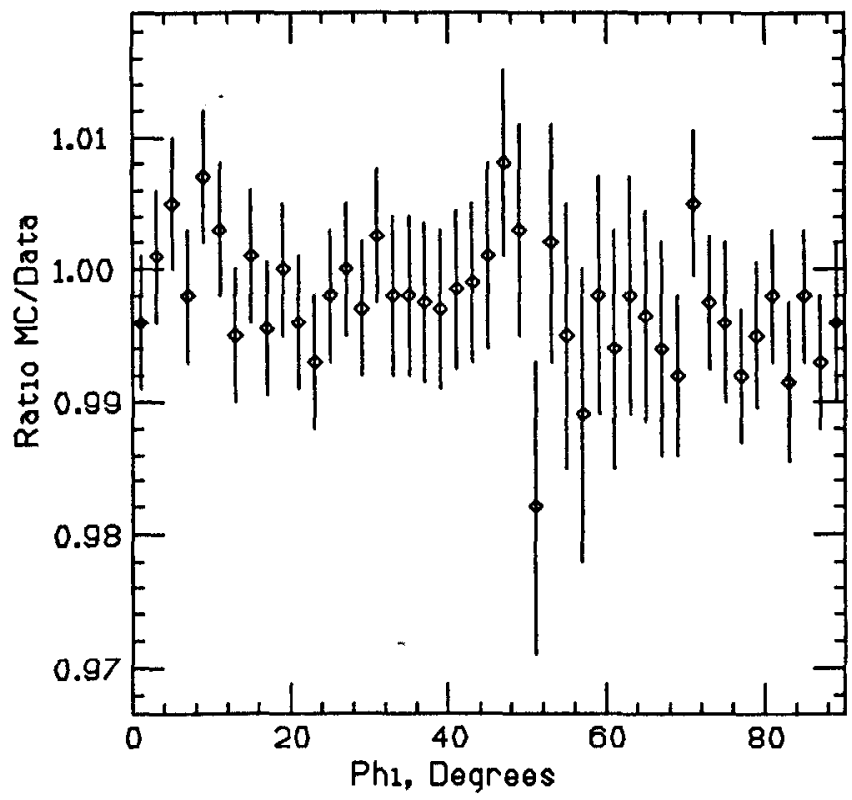

Figure 4.1. Ratio of average simulated lead-glass signal to average data as a function of $\phi$, obtained from simulation of kinematically fitted radiative Bhabhas.

Charged particles traversing the central PWC's ionize gas molecules, and the 
liberated electrons drift along the electric field lines to the sense wire. Near the sense wire the electrons begin to ionize other gas molecules and an avalanche is created, which provides signal amplification. If there are many charged particles, a saturation point is reached and the response becomes non-linear. In order to simulate this process, a signal proportional to the energy lost by each charged particle in the gas volume of the central PWC's is accumulated in AUSGAB. When the simulation of the event is complete, the signal in each PWC cell is corrected to include non-linear saturation effects. A comparison of the simulated central PWC signal with data has been performed using kinematically fitted radiative Bhabha events, and the ratio as a function of track energy is shown in fig. 4.2. Reasonably good agreement is obtained for tracks above $250 \mathrm{MeV}$; below this energy the fits are not very reliable. The ratio of Monte Carlo to data drops below 1 as the track energy increases; however for this analysis most tracks are in the range $.275-1$. GeV and are not much affected by the disagreement.

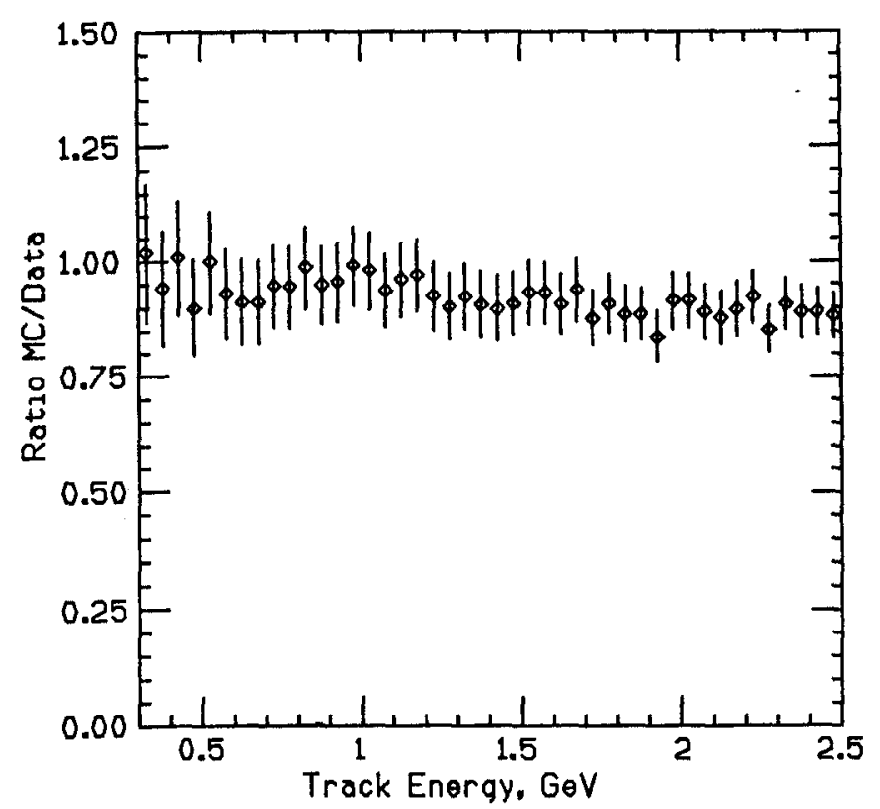

Figure 4.2. Ratio of average simulated central PWC signal to average data as a function of track energy, obtained from simulation of kinematically fitted radiative Bhabhas.

The energy deposited by charged particles in the central tracker is accumulated after applying a $\theta$-dependent correction taken from an analysis of real data. Charge 
division of the signal is obtained by dividing the signal into two parts proportional to the distance from each end. This results in a simulation which is more perfect than in reality, as it does not include the sometimes large systematic errors caused by offsets due to noise or cross-talk which affect the real data.

The veto scintillators surrounding the central tracker are simulated by accumulating the energy deposited by each charged particle, multiplied by an exponential attenuation factor. The signal is divided into two according to the distance of the track from the PMT's mounted on each end.

For the forward shower counters and forward PWC's, the electromagnetic shower is not fully simulated. Instead, look-up tables are used to simulate the response as a function of energy and position, reproducing the irregular pattern of radiation damage to the scintillator in the forward shower counters. This method saves CPU time, especially when the forward-going particle is a scattered electron with close to beam energy. The forward drift chambers are not simulated, and neither are the time-of-flight counters.

Detector noise is included in the simulation by overlaying the simulated events with real events logged out by the random trigger. Because most beam crossings do not result in any interaction, these random triggers represent the quiescent detector response with beam present in the storage ring. The random events have been luminosity weighted, with more events taken from periods with high beam currents and high luminosity.

\subsection{Trigger Simulation}

The ASP trigger has already been described in Chapter 2. The most important trigger for this analysis was the T6 trigger, also called the 'super trigger' because it has the lowest energy threshold in the lead-glass, about $0.7 \mathrm{GeV}$. The mass of the $\eta$ is $549 \mathrm{MeV}$, which is below this threshold. This results in a significant trigger inefficiency for $\eta \rightarrow \gamma \gamma$ events (about 50\%), and means that, the trigger must be very well understood. To achieve this, a detailed simulation involving threshold effects for every input to the trigger logic has been performed. 
The inputs to the trigger logic are discriminated signals from the lead glass, the forward shower counters, and the veto scintillators. From the lead glass alone there are 36 logical signals, obtained by discriminating the total lead-glass energy at four different levels, the quadrant sums at two different levels, the twenty layer sums and the sum of the back four layers in each quadrant. Each discriminator threshold may vary slightly from others set at the same level, and the rise may also vary. Over time the response remained very constant due to the uniform temperature maintained in the electronics building. -

To duplicate this threshold behavior, T7 triggers were selected. This trigger requires a very low threshold in the lead glass, about $0.3 \mathrm{GeV}$, in coincidence with significant energy in the forward shower counters on either side. The events thus selected are primarily radiative Bhabha events. This data set provides an unbiased sample with which to study the other lead-glass triggers, because the T7 threshold is so much lower than any of the others.

Using the T7 triggers, the threshold response for each trigger input was obtained as a function of raw PMT signal. These histograms were then stored as look-up tables. To determine whether a simulated event had fired the trigger, the simulated raw counts were summed and 'discriminated' using a random number generator and interpolating the look-up tables for each input. Once all trigger inputs had been simulated the trigger logic was applied, and the trigger arrays were filled just as they are for real data.

For the forward shower counters a different approach was necessary because the simulation of raw energy was not very accurate for these devices, due to an uneven pattern of radiation damage to the scintillator. Instead, the probability for the forward triggers to fire was calculated as a function of the energy and position of the incident particle. To do this, radiative Bhabha events with at least one forward track were selected in which the total lead-glass energy trigger had fired. This sample was therefore independent of the forward trigger. A kinematic fit was used for the trajectories of the forward tracks.

Simulation of the veto scintillator trigger bits was unnecessary because only the 
early VS cosmic trigger required them. Although the T6 trigger was vetoed if 3 or more veto scintillators were above threshold, this had a negligible effect on the trigger efficiency for events with 2 or fewer charged tracks. This was determined using a sample of events recorded with a special pre-scaled $\mathrm{T} 6$ trigger which did not have this veto. 


\section{5}

\section{Event Selection}

Approximately 30 million events recorded by the ASP detector were logged to tape. From this initial sample a few thousand events were selected for the final data sample. This reduction of four orders of magnitude was achieved in several stages.

The final state selected by this analysis has two photons in the lead glass calorimeter from the decay of an $\eta$ or $\eta^{\prime}$. The two photons are typically back to back in the azimuthal plane and acollinear in the plane containing the beamline. The electrons escape down the beampipe and are not observed. The total energy is much less than the total center-of-mass energy of $29 \mathrm{GeV}$. The specific criteria designed to select events with these distinctive characteristics are described in this chapter.

\subsection{Production First Pass}

All of the raw data was first processed by a filter designed to reject obvious junk, such as cosmic rays and beam-gas interactions, as quickly and efficiently as possible.

Cosmic ray events were rejected by exploiting the fact that they are distributed uniformly in position and time instead of originating at the interaction point in time with the beam crossing. If the event time was well measured, it was required to occur within $5 \sigma$ of the beam crossing. Events were rejected in which the lead-glass track closest to the origin passes more than $20 \mathrm{~cm}$ away, or in which the central PWC track closest to the origin is more than $20 \mathrm{~cm}$ from the origin in the xy plane. (The energy deposition pattcrn was required to be uniform, as expected from a minimum ionizing 
particle, for tracks which failed these cuts.) Beam-gas events and 'beam-burps' were eliminated by rejecting events with more than 80 tubes hit in the central tracker.

After rejecting identified cosmic rays and beam-gas events, a positive signature from each event was required in the form of at least one lead-glass cluster with more than $60 \mathrm{MeV}$ of energy, originating from the interaction point. To originate from the interaction point the track was required to have a distance of closest approach to the origin, $R=|z| \sin \theta$, of less than $30 \mathrm{~cm}$. This was a very loose cut and was designed to accept any event which was-even remotely interesting.

About 12 million events satisfied these criteria. They were then tracked and written out for further analysis. Diagnostic events, such as random triggers and VS cosmic triggers, were skimmed off separately for detector studies.

\subsection{Analysis First Pass}

In the first pass of this analysis, a very loose selection is made which includes charged events. In particular, events from the process $e^{+} e^{-} \rightarrow\left(e^{+} e^{-}\right) e^{+} e^{-}$, which resemble the $\eta \rightarrow \gamma \gamma$ topology, are retained for efficiency studies. Ten percent of the events on the production first pass tapes survive these cuts, described below.

The first requirement is that the event should be in the detector acceptance and have the correct multiplicity. At least two and not more than three lead-glass clusters with energy greater than $100 \mathrm{MeV}$ and with $\theta_{p}>20^{\circ}$ are required. Lead-glass clusters which are split between two quadrants, or small clusters which are fluctuations from a large cluster, are combined before determining the total. Projected theta is defined as

$$
\tan \theta_{p}=\frac{\sin \theta \times \max (|\cos \phi|,|\sin \phi|)}{\cos \theta} .
$$

Projected theta can be thought of as the polar angle, $\theta$, projected into the $\mathrm{xz}$ or $\mathrm{yz}$ plane. Using $\theta_{\mathrm{p}}$ to define the detector acceptance gives a square cutoff at the ends, corresponding to the box-like shape of the ASP detector.

The event is then required to have a lead-glass trigger, either $\mathrm{T} 1, \mathrm{~T} 2, \mathrm{~T} 3$ or $\mathrm{T} 6$, using the trigger definitions given in chapter 2 . This requirement is actually imposed when the data are collected, but it is re-iterated to define the trigger criteria explicitly 
and to apply the same requirements to simulated Monte Carlo data. In addition, a 'software trigger' is imposed by requiring each event to have at total lead-glass signal of at least 2400 counts, corresponding to approximately $700 \mathrm{MeV}$. The cut is defined in uncorrected lead-glass counts because this is what the trigger decision is based on. The efficiency of the 'super-trigger', the lead-glass trigger with the lowest threshold, is shown as a function of uncorrected counts in fig. 5.1; the efficiency is high and fairly flat above the software cut of 2400 counts.

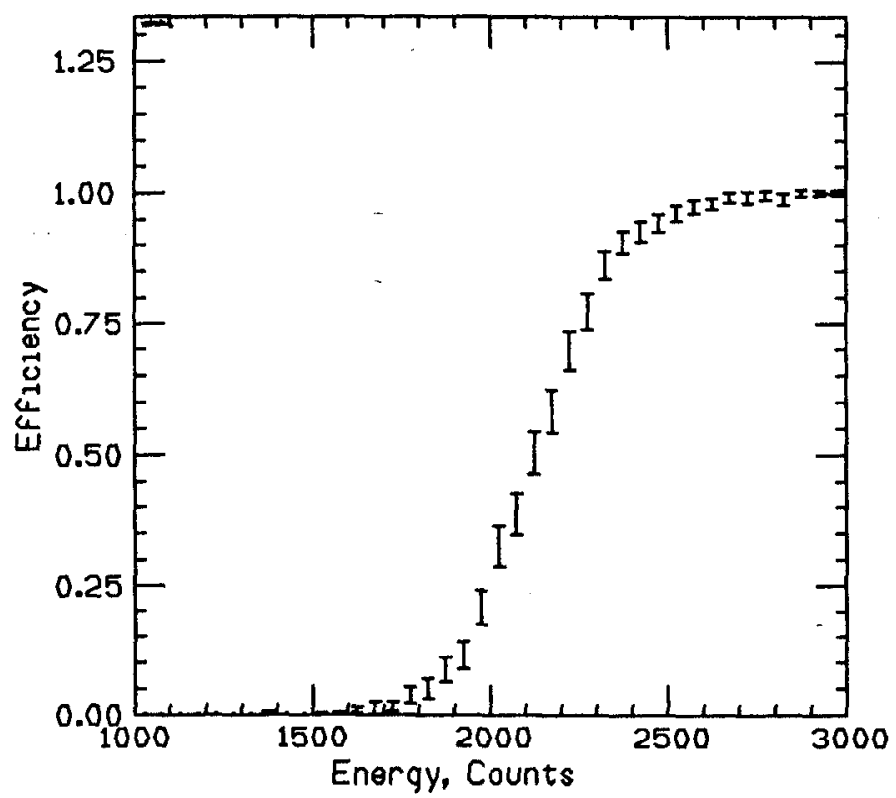

Figure 5.1. Efficiency for the T6 'super trigger' as a function of energy in raw counts. This threshold behavior has been included in the trigger simulation. The software trigger cut is placed at 2400 counts.

A very loose cut on the net transverse momentum is then made, by calculating the thrust axis of the event in the azimuthal plane, dividing the event into two hemispheres along the line perpendicular to this axis, and requiring at least one leadglass cluster in each hemisphere. This requirement rejects many beam-gas interactions and cosmic ray events in which all the energy is deposited on one side.

The inner forward shower counters are required to have less than $150 \mathrm{MeV}$ deposited in either side and the outer forward shower counters are required to have less than $300 \mathrm{MeV}$. These cuts reject tagged events and events with extra forward tracks. Bhabha events and $e^{+} e^{-} \rightarrow \gamma \gamma$ events are eliminated by requiring an acollinearity of 
at least $10^{\circ}$ for events with a total energy greater than $20 \mathrm{GeV}$.

A large fraction of the events remaining in the data sample at this stage are cosmic rays. Cosmic rays uniformly populate a 3 -dimensional volume whose axes are the distance of closest approach to the beamspot in $\hat{z}$, as measured by the lead glass, the distance of closest approach in the xy plane, as measured by the central PWC, and the event time. The first two quantities have already been used to reject cosmic rays in the production first pass. Those cuts were fairly loose, so we can tighten them up without sacrificing efficieney for signal events.

In the production first pass, at least one track was required with a distance of closest approach to the origin in $\hat{z}$ of less than $30 \mathrm{~cm}$. This cut is now applied to all lead-glass tracks for which $z$ is well measured. (Merged tracks which are split between two quadrants are not included because the $z$ reconstruction is not always very accurate in this case.)

Any event with a minimum-ionizing central PWC track which passes more than $10 \mathrm{~cm}$ from the interaction point in the xy plane is also rejected. This is more stringent than the $20 \mathrm{~cm}$ cut already applied. The central PWC track must have at least three layers, a narrow width and a uniform energy deposition pattern to be identified as minimum ionizing.

At this stage, it is useful to look at the liming distribution of the events which have passed all the cuts so far. The lead-glass timing information is only reliable for quadrants with at least $150 \mathrm{MeV}$. The time is corrected for the distance travelled from the interaction point to the lead-glass calorimeter and for the propagation time of the Cerenkov light to the PMT, using the track parameters. The resulting distribution, shown in fig. 5.2, is a Gaussian centered on 0.0 ns with $\sigma=2.2$ ns on a flat background. This flat background indicates that there are still cosmic rays present.

To reject most of the remaining cosmic ray background, the time-of-flight counters are used to identify cosmic rays which pass through the TOF before entering the lead glass. The TOF hit with the best match in $z$ and in $\phi$ is assigned to each lead-glass track. A scatterplot of the lead-glass time versus the TOF time is shown in fig. 5.3; the TOF time is corrected for the flight from the origin. A diagonal band of 


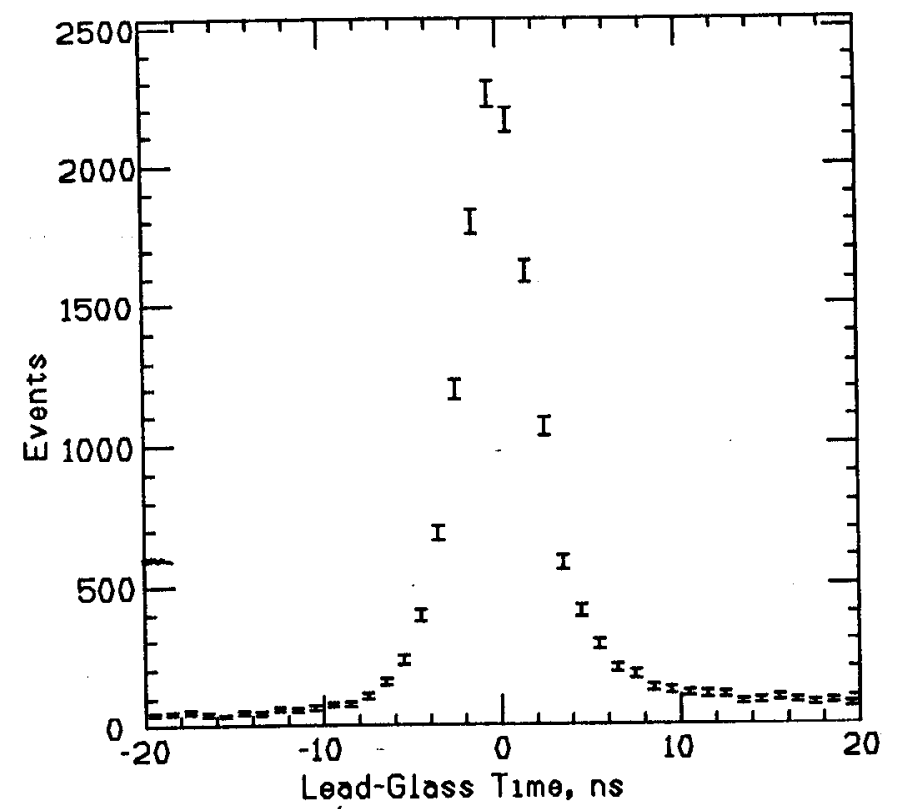

Figure 5.2. Lead-glass quadrant time, corrected for the distance of the track from the PMT. A gaussian with $\sigma=2.2 \mathrm{~ns}$ is scen on a flat background due to cosmic rays.

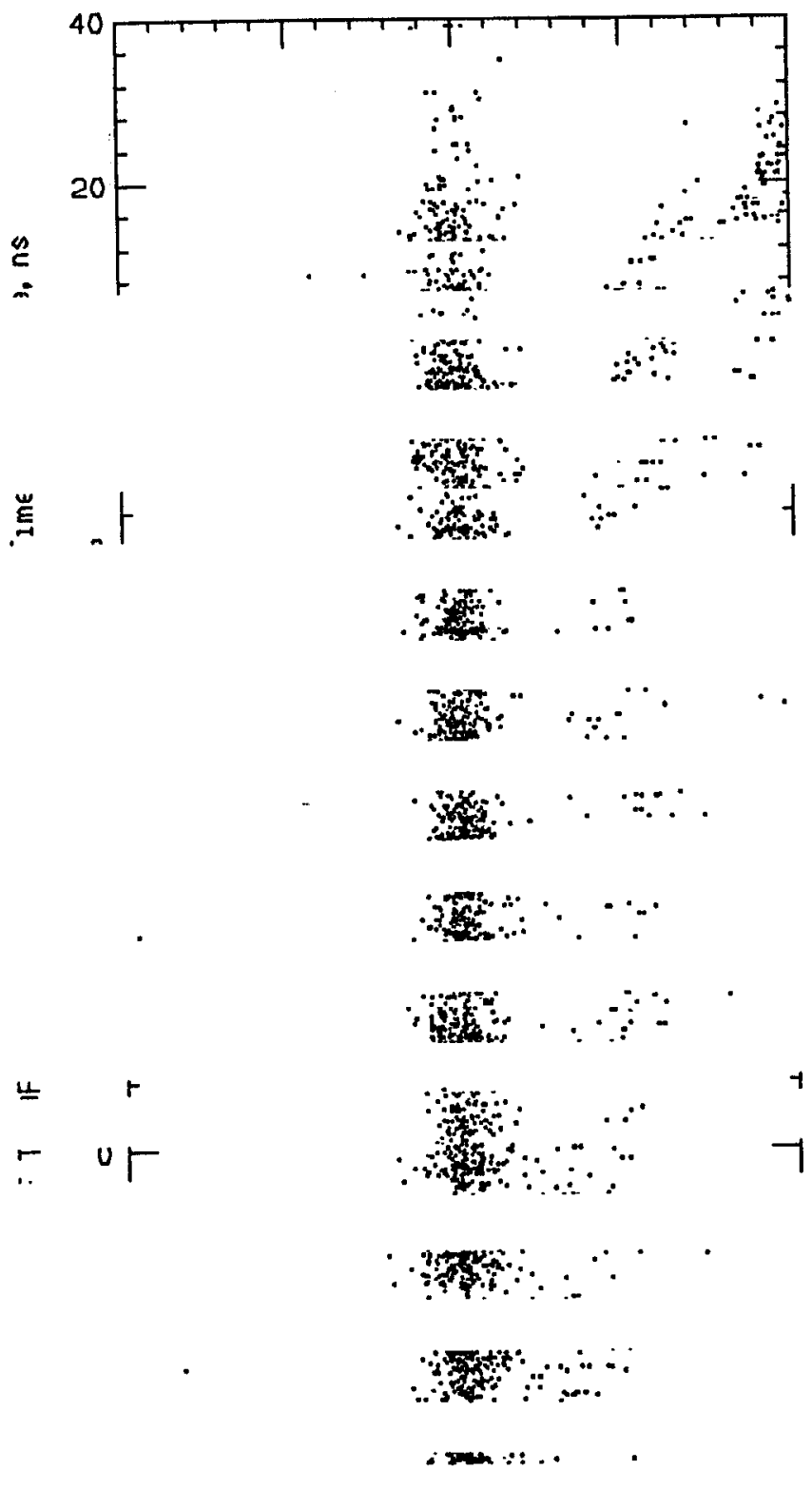




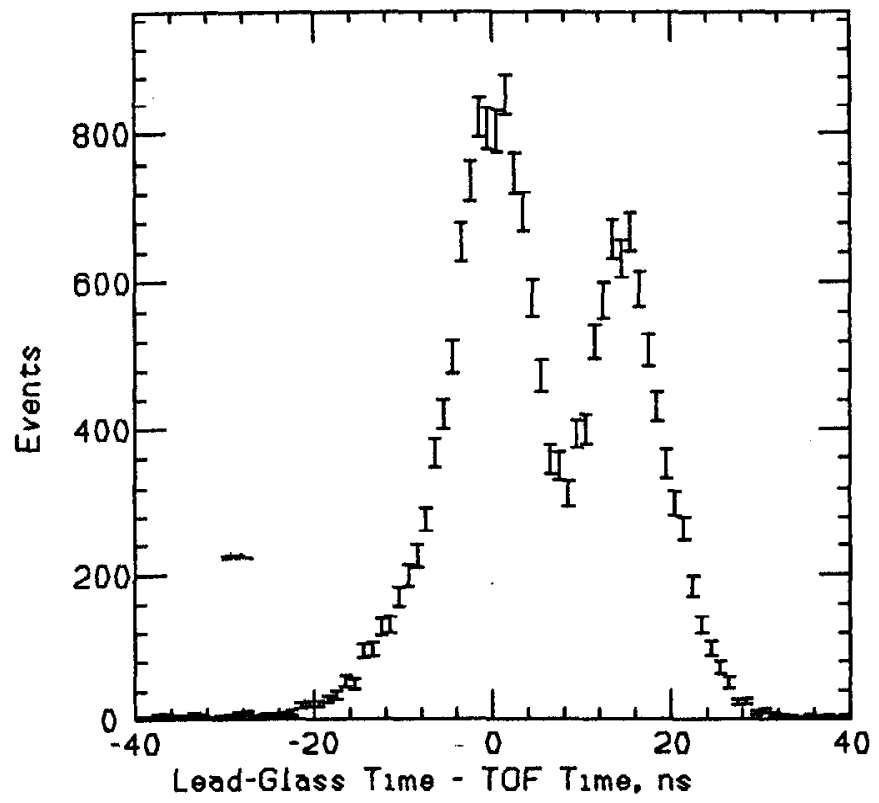

Figure 5.4. Lead-glass time - TOF time. The double peak is due to contamination from cosmic rays. Events with a lead-glass time more than $8 \mathrm{~ns}$ greater than the TOF time are rejected.

than $8 \mathrm{~ns}$ (and less than $40 \mathrm{~ns}$ ) after the TOF time.

\subsection{Final Event Selection}

At this stage all events are retracked. The reason for this time-consuming procedure is that the general-purpose tracking code is not optimized for the final states of interest. In particular, the energy threshold in the central PWC tracking routine is set too high for the small signals deposited by a photon of a few hundred $\mathrm{MeV}$. The lead-glass cluster finding algorithm was also modified to make it more sensitive to overlapping clusters, such as those from the decay of a $\pi^{\circ}$ into two photons. This was achieved at the cost of biasing the cluster-finding to the origin.

The retracked events were required to have two lead-glass tracks in the detector acceptance. After identifying the two most energetic tracks, any lead-glass signals which were not associated with these two tracks were accumulated and counted as unassociated energy; the total unassociated energy was required to be less than 25 $\mathrm{MeV}$. Because the lead glass was so quiet, this rather restrictive cut was quite efficient. Both lead-glass tracks were then required to have a $\phi$ measurement, either by association with a central PWC cluster or by reconstruction of $\phi$ from merging two 
clusters separated by quadrant boundaries.

Next the event was required to be neutral. This was done in a series of cuts, each more restrictive than the last. To start with, any event with a track in which a leadglass clustcr was linked to a central-tracker track was rejected. The tracking requires that the central tracker $\theta$, obtained from charge division, agree with the value of $\theta$ reconstructed in the lead glass. This matching can fail due to mis-measurement in either system. Because there is such a large background from two-photon production of charged lepton pairs, additional check is made for any central tracker hits which are close to the lead glass track in azimuth. If 3 or more central tracker planes have hits within $30^{\circ}$ in $\phi$, including the first or second plane, the track was classified as charged. A final criteria was that there be no more than one reconstructed central tracker track in the event.

Minimum-ionizing tracks were then identified using a careful analysis of layer energy deposition patterns. The energy in each layer is corrected for attenuation and a match between the shower shape in the lead glass and the central PWC systems is required. This cut is aimed primarily at cosmic rays which have evaded all previous efforts to weed them out, in particular those which pass close to the origin but were not identified as charged.

The remaining background is mostly from two-photon production of the $f_{2}(1270)$, a tensor meson which is copiously produced and decays into $\pi^{\circ} \pi^{\circ} 28 \%$ of the time. The $\pi^{\circ}$ 's may appear like two photons if they decay asymmetrically or if the photons overlap. Shower shape cuts were applied to reduce this background. The lead-glass shower shape was defined as the second moment of the track, constructed by summing up the square of the distance of each lead-glass block from the trajectory of the track in the $\mathrm{xz}$ or yz plane, $D_{i}$, weighted by its energy, $E_{i}$ :

$$
M_{W^{2}}=\frac{1}{\sum_{i} E_{i}} \sum_{i} E_{i} D_{i}^{2}
$$

This quantity was larger for wide showers due to overlapping photons from the decay of a $\pi^{\circ}$, as seen in fig. 5.5. Both tracks were required to have lead-glass cluster width 


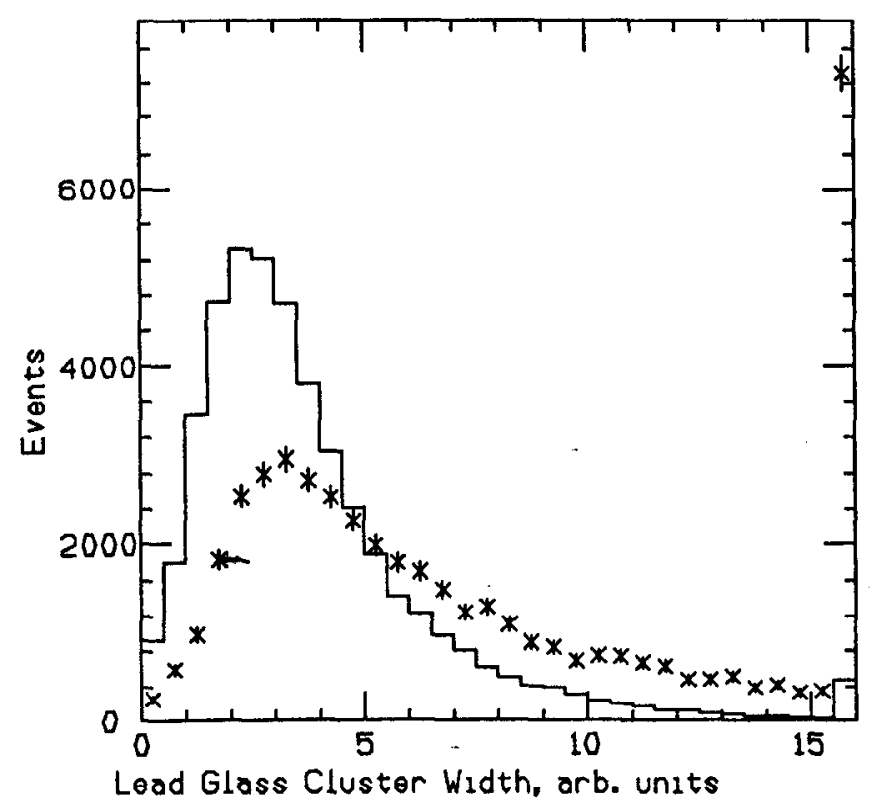

Figure 5.5. Lead-glass cluster width for photons from Monte Carlo $\eta \rightarrow \gamma \gamma$ events (histogram) and from Monte Carlo $f_{2} \rightarrow \pi^{\circ} \pi^{\circ}$ events (X's). Photon candidates with a lead-glass width greater than 8 . were rejected.

less than 8 .

A similar cut was made using the central PWC hits, summing over the square of the distance from the track to each CPWC hit in the xy plane, weighted by the CPWC signal. This CPWC shower shape cut was orthogonal to the lead-glass shower shape cut, because a $\pi^{\circ}$ which overlapped in the plane of the lead glass was likely to appear as two photons in the orthogonal plane of the CPWC.

Finally, the event was required to have a net transverse momentum less than $300 \mathrm{MeV}$. This is consistent with the requirement that there be no tag above 21 $\mathrm{mr}$, assuming only one electron is appreciably deflected. The $\mathrm{p}_{\mathrm{t}}$ distribution of data events passing all other cuts is shown in fig. 5.6 ; it falls off at $300 \mathrm{MeV}$ as expected.

The invariant mass distribution of the final event sample is shown in fig. 5.7. Clear peaks at the $\eta$ and $\eta^{\prime}$ mass are seen on a smooth background concentrated above $1 \mathrm{GeV}$. This background is discussed in the next chapter. 


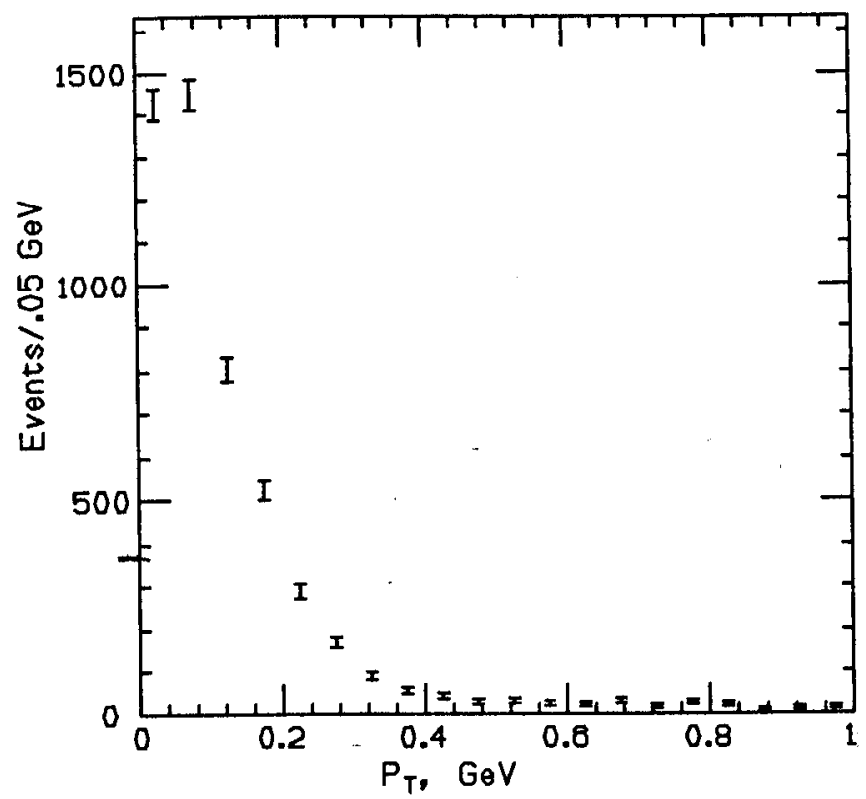

Figure 5.6. $p_{t}$ distribution of data events passing all other cuts. Events with a $p_{t}$ greater than $.3 \mathrm{GeV}$ were rejected.

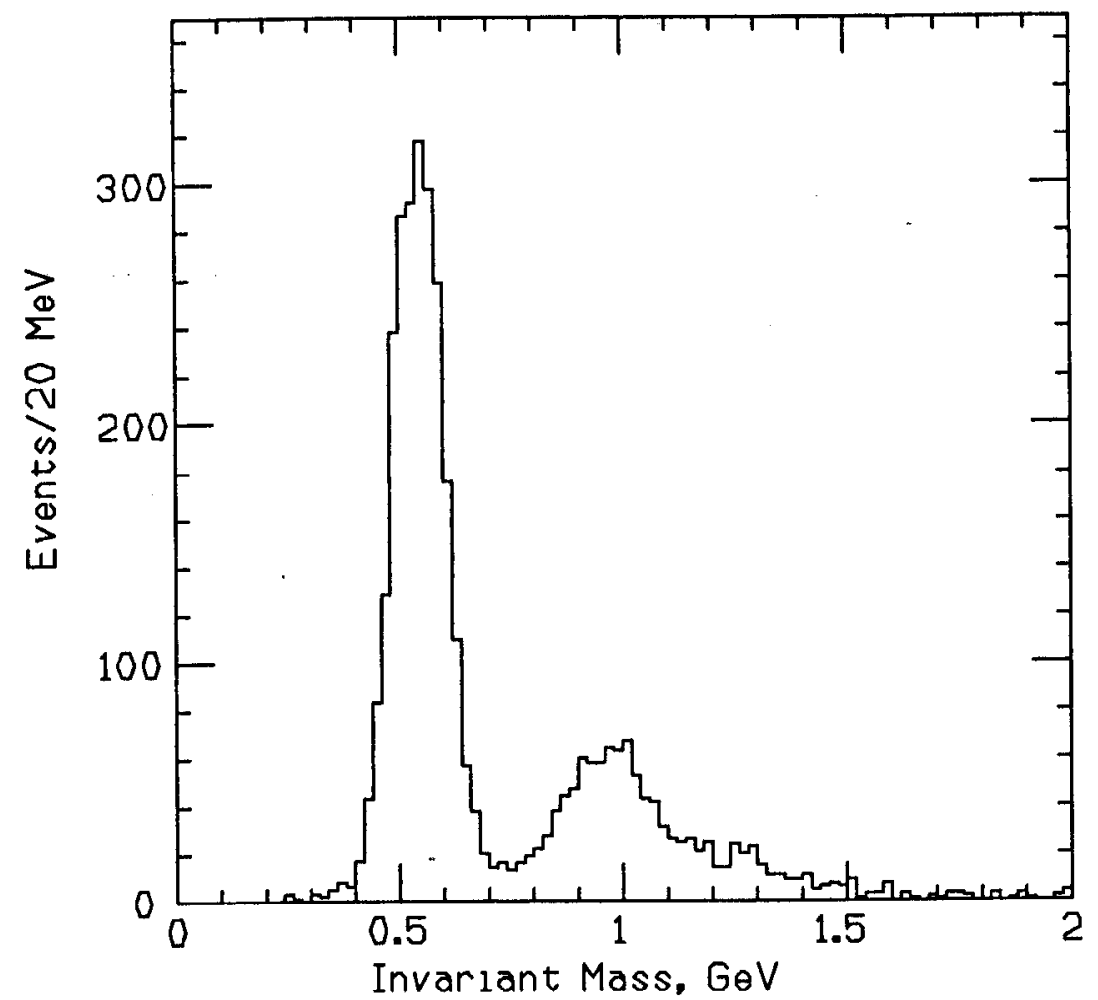

Figure 5.7. Invariant mass distribution of final data sample. Peaks are seen at the $\eta$ and $\eta^{\prime}$ masses. 
6

\section{Background Calculations}

\subsection{Sources of Background Events}

Events from the decays $\eta \rightarrow \gamma \gamma$ and $\eta^{\prime} \rightarrow \gamma \gamma$ have a distinctive signature, as discussed in the introduction to the previous chapter. Background events which can fake this signature can be divided into two general categories: those which originate from beam-beam interactions and those which do not. In the former category are fourth-order QED processes with two photons in the final state, as well as two-photon production of resonances with decay modes that include photons. Another source of background could be due to the misidentification of charged particles, in reactions such as $e^{+} e^{-} \rightarrow\left(c^{+} e^{-}\right) e^{+} e^{-}$. These processes are all calculable, so it is straightforward to estimate the background from each one. In the second category are cosmic rays and beam-gas interactions. We can use the data to estimate the expected contribution from these sources. The details of all background estimates are presented in this chapter.

\subsection{Fourth-Order QED Backgrounds}

The QED diagrams which contribute to the process $e^{+} e^{-} \rightarrow\left(e^{+} e^{-}\right) \gamma \gamma$ include the bremsstrahlung diagrams of fig. 6.1 as well as the box diagram of fig. 1.1a, inserted into the usual two-photon diagram. (Other diagrams which do not contribute to the untagged topology with both photons at wide angles have been neglected.) The 
bremsstrahlung diagrams have been calculated using a Monte Carlo based on the equivalent photon approximation. ${ }^{(34)}$ The total cross section is large, but requiring both photons to be in the lead-glass acceptance with an invariant mass greatcr than $0.3 \mathrm{GeV}$ results in an accepted cross section of only $.008 \pm .003 \mathrm{pb}$, yielding less than 1 event in the data sample of $108 \mathrm{pb}^{-1}$. These diagrams have therefore been neglected.
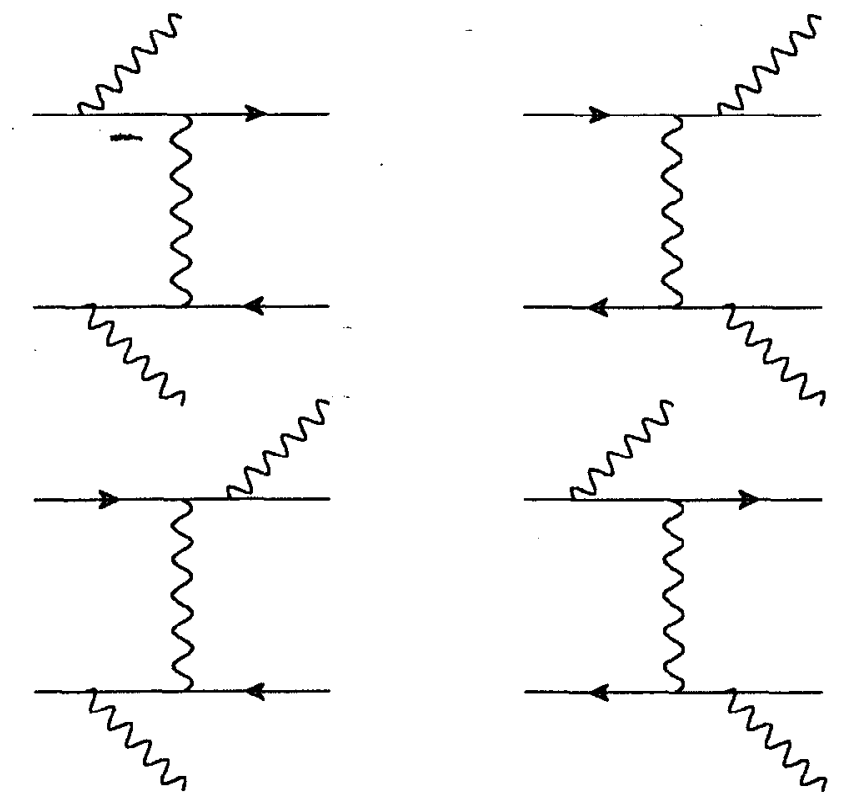

Figure 6.1. Feynman diagrams from the untagged process $e^{+} e^{-} \rightarrow\left(e^{+} e^{-}\right) \gamma \gamma$. Only bremsstrahlung diagrams are shown.

The two-photon process involving the box diagram is actually sixth-order in the QED coupling constant $\alpha$. The cross section has been estimated using the equivalent photon approximation and an approximation for non-resonant photon-photon scattering which is valid in the limit $\frac{\omega}{m_{e}} \rightarrow \infty$, where $\omega$ is the energy of the incident pholons. ${ }^{(35)}$ For a minimum invariant mass of $0.3 \mathrm{GeV}$, the total cross section is 3.5 $\mathrm{pb}$. Requiring both photons be in the detector acceptance reduces the cross section to $.2 \mathrm{pb}$, and after the trigger and event selection criteria have been applied it is further reduced to $.04 \mathrm{pb}$, yielding less than 5 background events. This is negligible compared to the expected signal.

Two-photon production of electron pairs, $e^{+} e^{-} \rightarrow\left(e^{+} e^{-}\right) e^{+} e^{-}$, is a copions snurce of events. This reaction proceeds through the two-photon diagram of fig. $1.2, \ldots \ldots l$ has 
the same topology as the signal. The only difference is that the events are charged; otherwise electrons and photons are indistinguishable in the ASP detector.

For charged particle identification we rely on the central tracker, which has five layers of tracking. To determine the efficiency of the central tracker, cosmic ray events from the early VS cosmic trigger were used. The penetrating cosmic radiation consists primarily of muons which should leave charged tracks if they pass through the central tracker. To identify such events, a track in the calorimeter was required with at least four layers hit in the central PWC system, pointing to within $3 \mathrm{~cm}$ of the origin in the transverse plane. This insured that the tracks did indeed pass through the central tracker and would leave a pattern of hits similar to that from tracks originating from the origin.

To see if the central tracker had registered the track, the azimuthal angle of the central PWC track was calculated with a constraint to the origin and a search was made for central tracker tracks which were within $\pm 30^{\circ}$ in $\phi$. If none was found, individual central tracker hits within the same angular region were identified, and the same requirements made in the data analysis were applied to determine if the track was charged. (At least three hits, with at least one in the first or second layer.) Of the 19,775 cosmic ray tracks subjected to this test, 78 were found to be neutral. They were all scanned, and of these 48 were found to be due to central tracker inefficiency. The others were tracks which did not pass through the central tracker and had been mis-measured by the central PWC system, or tracks which passed vertically between central tracker tubes in three of the five layers. (This is not possible for tracks which originate from the origin.) From this result the calculated central tracker efficiency is $99.76 \%$.

For an event with two charged tracks to be identified as neutral, the central tracker would have to fail twice, so the inefficiency for this is $.0024^{2}$, or $.0006 \%$. From an analysis of $e^{+} e^{-} \rightarrow\left(e^{+} e^{-}\right) e^{+} e^{-}$events in the data, the number of events which pass all event selection criteria for the $\gamma \gamma$ final state but are identified as charged is approximately 220,000 . Thus the background due to mis-identification of electron pairs from two-photon interactions is 1.3 events and can be neglected. 
The process $e^{+} e^{-} \rightarrow\left(e^{+} \gamma\right) e^{-} \gamma$, where an electron and a photon are produced at wide angles, has a much smaller cross-section, but only one charged track would have to be misidentified as neutral for it to contribute as background. The cross section for this process was calculated, requiring one electron and one photon to be in the detector acceptance with an invariant mass greater than $.3 \mathrm{GeV}$, with a result of $320 \pm 5 \mathrm{pb}$. Taking the central tracker efficiency into account yields 84 events $e^{ \pm} \gamma$ events in $108 \mathrm{pb}^{-1}$ in which the charged track is misidentified as neutral. To determine the event selection efficiency, events of this type were generated and simulated, and the trigger requirement and all event selection criteria were imposed. This reduced the expected background to 14.9 events with an invariant mass less than $2 \mathrm{GeV}$; the invariant mass distribution is shown in fig. 6.2. There are $2.9 \pm .8$ events in the $\eta$ mass region $(.4-.7 \mathrm{GeV})$ and $7.8 \pm 1.2$ events in the $\eta^{\prime}$ mass region $(.7-1.2 \mathrm{GeV})$;

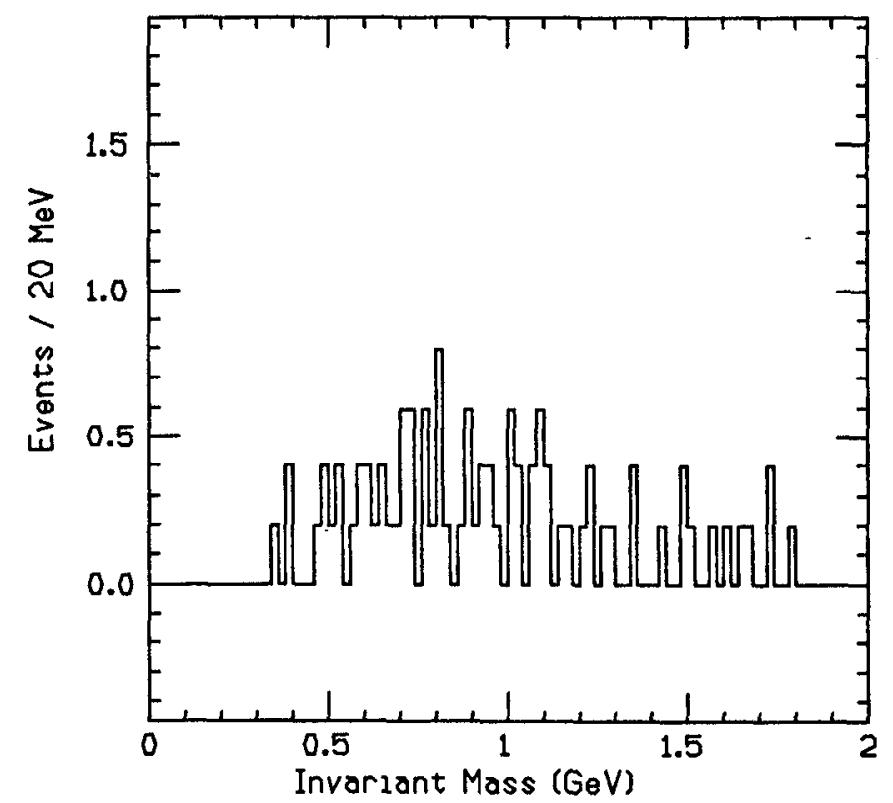

Figure 6.2. Invariant mass distribution of $e^{ \pm} \gamma$ events passing all event selection criteria, normalized to data luminosity.

\subsection{Two-photon backgrounds}

Two-photon processes which can contribute background events include $\gamma \gamma \rightarrow$ $f_{2}(1270)$, with subsequent decay of the $f_{2}$ into $\pi^{\circ} \pi^{\circ}$. There is also a small amount of two-photon continuum $\pi^{\circ} \pi^{\circ}$ production at small invariant masses. The $a_{2}(1320)$ 
is another resonance produced in two-photon interactions; it contributes to the background in the decay mode $\eta \pi^{\circ}$. These backgrounds are all evaluated in this section.

The $f_{2}(1270)$ is a tensor meson, $J^{P C}=2^{++}$, which has a total width of 176 $\mathrm{MeV}$ and a $28 \%$ branching ratio to $\pi^{\circ} \pi^{\circ}$. It is copiously produced in $\gamma \gamma$ interactions. The segmentation of the lead-glass calorimeter is not fine enough to distinguish $\pi^{\circ}$ 's from photons with $100 \%$ efficiency, allowing some fraction of these events into the final data sample. To accurately estimate this fraction, a sample of almost 90,000 $f_{2} \rightarrow \pi^{\circ} \pi^{\circ}$ events corresponding to $220 \mathrm{pb}^{-1}$, or slightly more than twice the luminosity of the data, was generated using a Monte Carlo event generator based on the equivalent photon approximation. The angular distribution was generated assuming $100 \%$ helicity 2 . The total cross section of $399.5 \pm .7 \mathrm{pb}$ was obtained from an exact calculation, ${ }^{(36)}$ which differed by only $5 \%$ from the EPA result. The events were passed through the detector simulation, trigger and event selection routines; the total detection efficiency was $1.0 \%$. The invariant mass distribution of the events passing all cuts, normalized to the data luminosity, is shown in fig. 6.3. The peak is centered below the $f_{2}$ mass; this is due both to the photon flux, which is falling like $\frac{1}{s}$, and to the fact that this is a $4 \gamma$ final state which is required to look like a $2 \gamma$ final state, so some low-energy photons may be undetected.

The expected number of $f_{2}$ events in $108 \mathrm{pb}^{-1}$ is $123 \pm 15$, where the error is statistical only. There is an additional, and potentially large, systematic error due to uncertainty in the Monte Carlo efficiency calculation and in the helicity distribution. Very few events contribute to the $\eta$ signal region below .7 Gev, but there is a significant contribution to the $\eta^{\prime}$ mass region around $.95 \mathrm{GeV}$. This background must be determined by a fit to the data, with the mass and width of the $f_{2}$ constrained to those determined by a Breit-Wigner fit to the Monte Carlo distribution. This is discussed in further detail in chapter 8.

In addition to resonance production of the $\pi^{\circ} \pi^{\circ}$ final state there is a small continuum contribution due to final state interactions. This has recently been measured by the Crystal Ball collaboration, see fig. 6.4, and their result has been used to estimate the background contribution to this analysis. Events were generated using the 


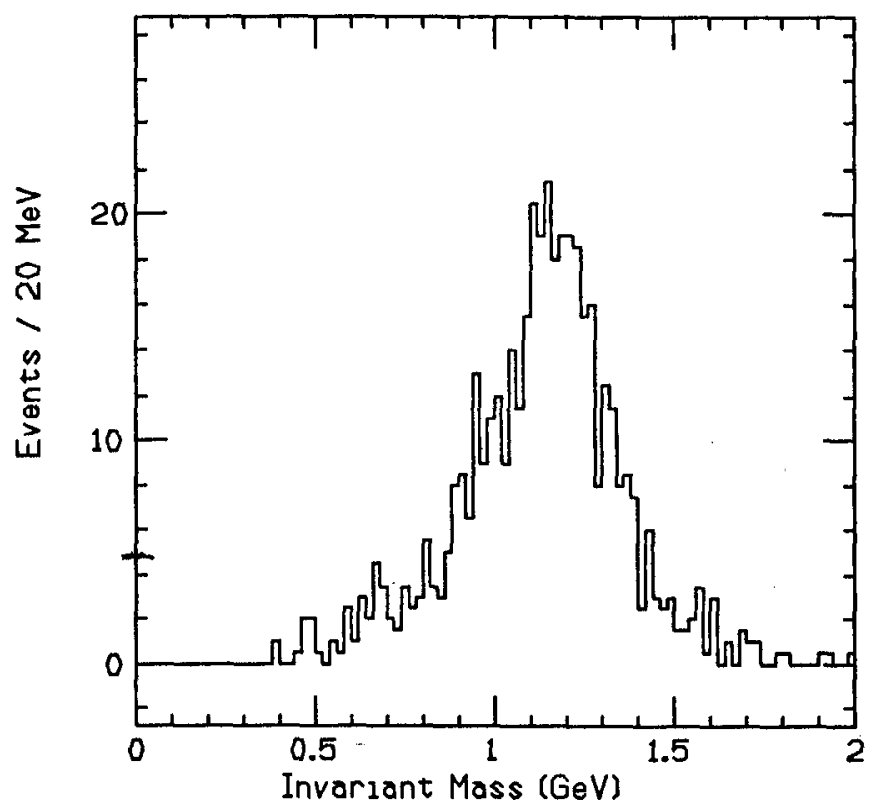

Figure 6.3. Invariant mass distribution of $f_{2}(1270) \rightarrow \pi^{\circ} \pi^{\circ}$ events passing all event selection criteria, normalized to data luminosity.

equivalent photon approximation and a lookup table for the $\gamma \gamma \rightarrow \pi^{\circ} \pi^{\circ}$ cross section taken from fig. 6.4. Above an invariant mass of $.8 \mathrm{GeV}$ the $f_{2}(1270)$ contribution dominates, so the continuum cross section was assumed to fall like $\frac{1}{s^{3}}$, the theoretical expectation. The events were passed through the detector simulation and the event selection routines. The total production cross section was $414 \mathrm{pb} ; 584 \mathrm{pb}^{-1}$ were generated, more than five times the data luminosity. The invariant mass spectrum of events passing all cuts is shown in fig. 6.5. The expected number of background events from this source is estimated to be $44 \pm 22,37$ of them in the $\eta$ mass region. An error of $50 \%$ has been assigned because of the limited information available on the shape and magnitude of the continuum $\gamma \gamma \rightarrow \pi^{\circ} \pi^{\circ}$ cross section.

To estimate the contribution from the $a_{2}(1320)$, events were generated using the equivalent photon approximation. A total luminosity of $220 \mathrm{pb}^{-1}$ was generated in the decay mode $a_{2} \rightarrow \eta \pi^{\circ}$, the mode most likely to contribute. From this the expected number of $a_{2}$ events was determined to be $9 \pm 2$, with an invariant mass distribution centered near $1.1 \mathrm{GeV}$. There are no events in the $\eta$ mass region, and a total of $6 \pm 1.7$ events in the $\eta^{\prime}$ mass region. 


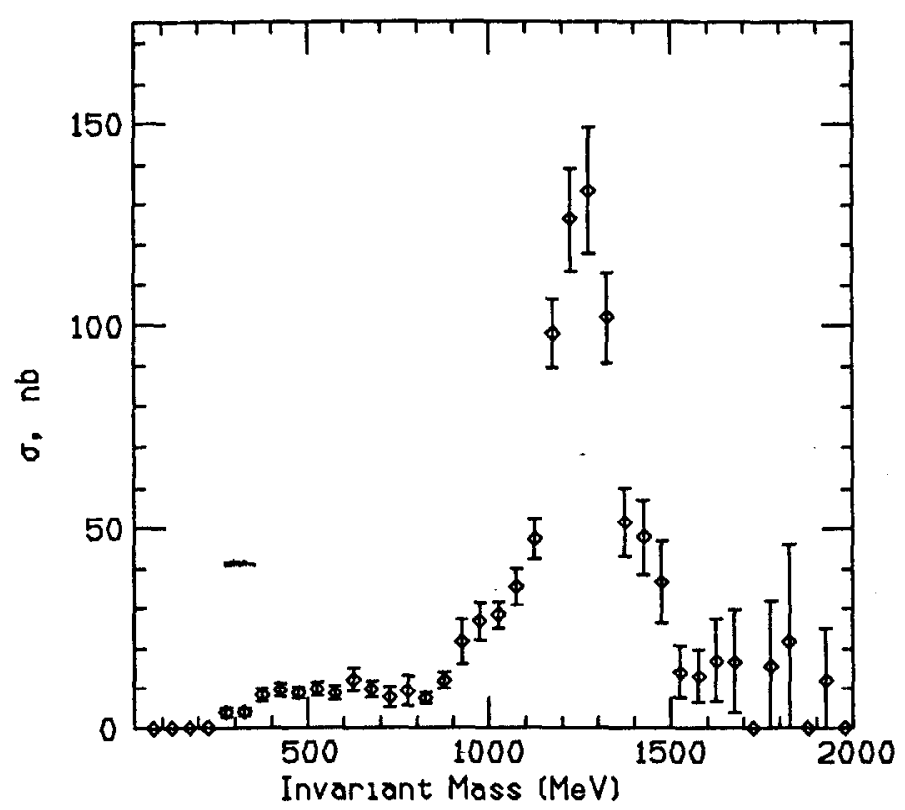

Figure 6.4. Invariant mass distribution of $\pi^{\circ} \pi^{\circ}$ events measured by the Crystal Ball, for events with $\left|\cos \theta^{*}\right|<.8$. Continuum production below the $f_{2}(1270)$ resonance is evident.

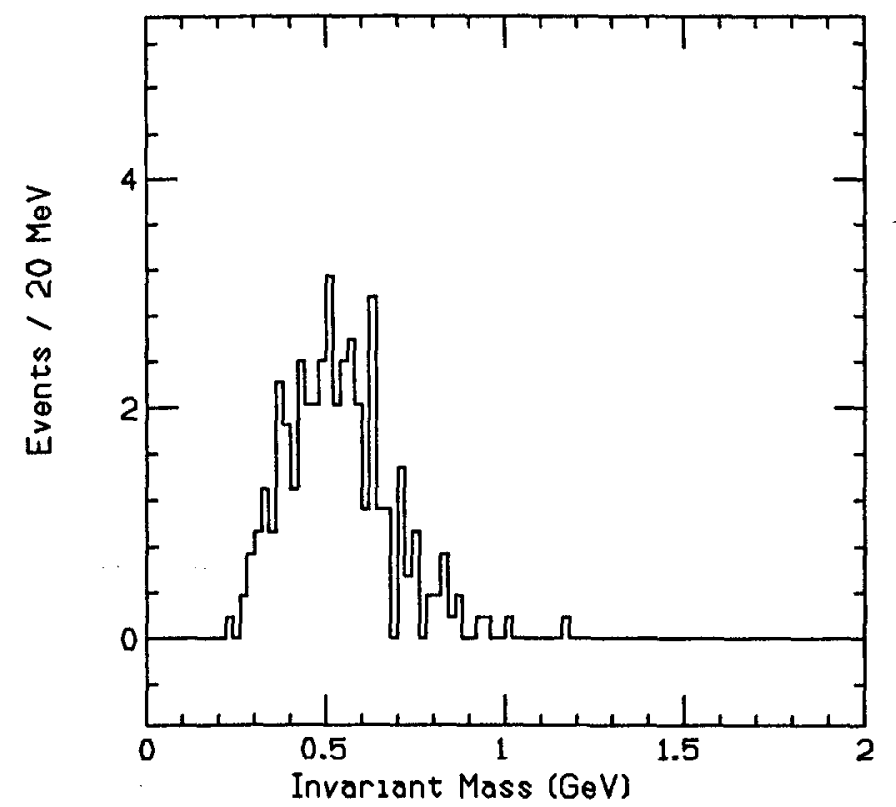

Figure 6.5. Invariant mass distribution of $\pi^{0} \pi^{0}$ continuum events passing all cuts.

\subsection{Cosmic Rays}

Cosmic rays are continually bathing the detector with minimum-ionizing particles. They are spatially and temporally uncorrelated with the beam crossing, and most deposit too little energy in the lead-glass calorimeter to trigger the detector. Yet they are still one of the most persistent and difficult backgrounds. 
The cuts used to reject cosmic rays were described in chapter 5. These included cuts on the distance of the track from the origin in the plane transverse to the beam and the plane including the beam, a cut on the difference between the lead-glass time and the TOF time, and rejection of events with tracks which appear to be minimum ionizing. The cuts against charged events also reject many cosmic ray events.

However, no cut has been made on the lead-glass time by itself, with the exception of the $5 \sigma$ timing cut made in the production first pass. In that cut, at least 440 $\mathrm{MeV}$ was required in one lead-glass quadrant; any event which did not satisfy this requirement had its time set to 0 , so the cut was not applied to many cosmic rays. In addition, only slewing corrections were applied, and no corrections for the particle trajectory or light propagation time were applied. The calculated $\sigma$ was therefore larger than that calculated for the final data set in which these corrections were applied.

To determine the shape of the timing distribution for cosmics which passed the $5 \sigma$ timing cut, events were identified as cosmics using the difference between the leadglass and TOF times. (The lead glass time was required to be at least $10 \mathrm{~ns}$ greater than the TOF time, to reduce contamination from annihilation events.) These events passed all cuts up to the TOF cut; further cuts were not applied in order to increase the statistics. The timing distribution for these cosmic ray cvents is shown in fig. 6.6; a flat distribution extending beyond $\pm 20 \mathrm{~ns}$ is observed, with an enhancement for times within \pm 10 ns due to the $5 \sigma$ timing cut. The ratio of all events in fig. 6.6 to those events with $20>|t|>10 \mathrm{~ns}$ is $3.6 \pm .3$. By contrast, the timing distribution of events in the final data sample, shown in fig. 6.7, is sharply peaked about 0 . There is one event in the data sample with $20>|t|>10 \mathrm{~ns}$, so the predicted number of events due to cosmic rays is 3.6 (or less than 13.9 events at the $90 \%$ C.L.), and has therefore been neglected.

\subsection{Beam-Gas Interactions}

The electrons and positrons circulating in the PEP storage ring may interact with residual gas molecules in the vacuum pipe. Although the pressure at the IP 


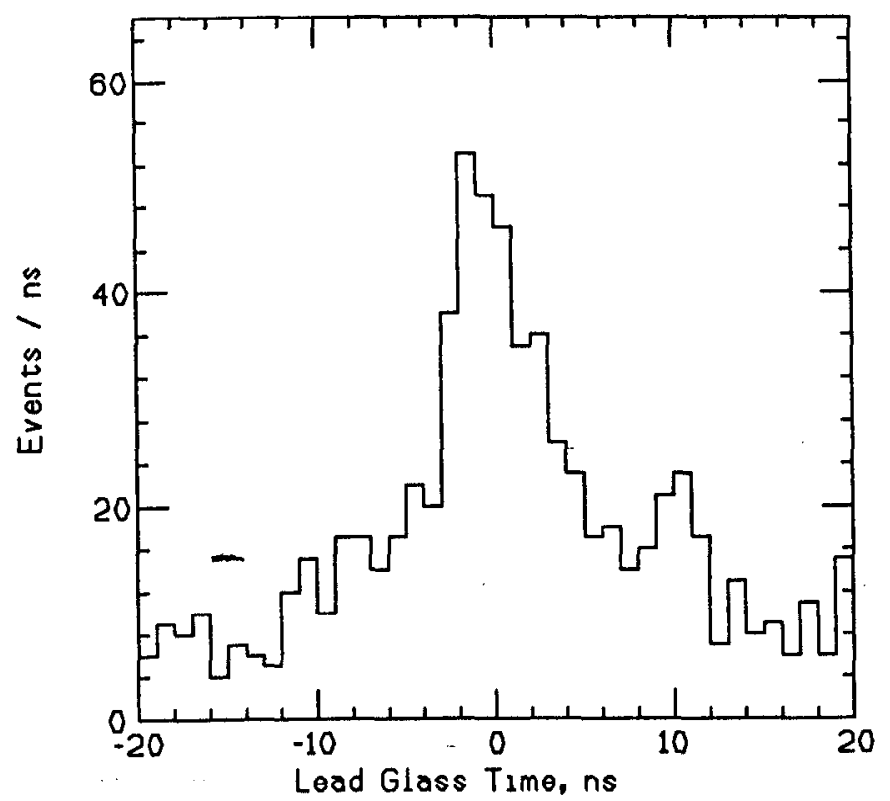

Figure 6.6. Event time for cosmic ray events which passed the $5 \sigma$ timing cut. The peak is an artifact of the cut.

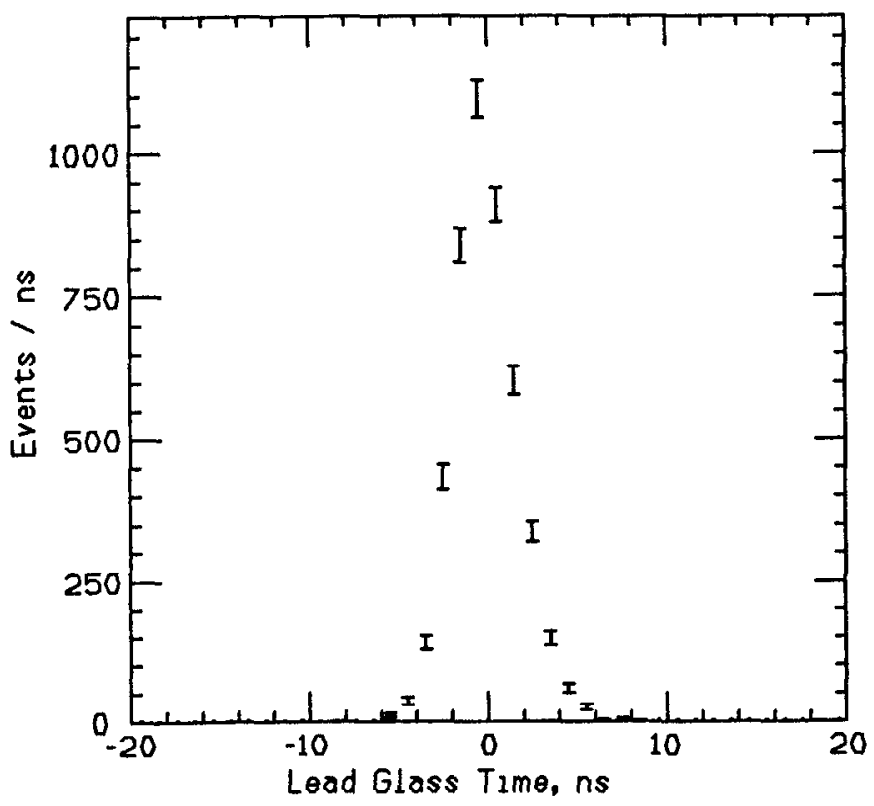

Figure 6.7. Event time for all events in final sample.

was typically $10^{-9}$ Torr or less, that works out to $3.2 \times 10^{7}$ particles per $\mathrm{cm}^{3}$. These beam-gas collisions typically produce debris at very low angles, but occasionally the collision products may enter the detector.

A particularly dangerous background comes from photo-production of $\eta$ mesons, resulting in an invariant mass distribution similar to that of two-photon production 
of $\eta$ 's. This occurs when an electron or photon emits a bremstrahlung photon, which interacts with the hadronic field of a nucleon to produce a nucleonic resonance, which may then decay to a nucleon and an $\eta$ meson. These events can be distinguished from two-photon interactions by the fact that they are produced uniformly in $\hat{z}$. They also have a flatter $\mathrm{p}_{\mathrm{t}}$ distribution, and the recoiling nucleon may interact in the detector.

To estimate the contribution from this source, two different methods were used, giving comparable results. In the first method, the $z$ distribution of events in the final data sample was used to timate the beam-gas background. The second method was to calculate the expected number of events based on the measured $\eta$ photo-production cross section.

The $\mathrm{z}$ distribution of events in the final data sample with invariant mass less than $.7 \mathrm{GeV}$ is shown in fig. 6.8 , together with the $\mathrm{z}$ distribution of Monte Carlo $\eta$ events originating from the IP, normalized to the same number of events. Requiring both tracks to have $\mathrm{z}$ greater than $+10 \mathrm{~cm}$ or less than $-10 \mathrm{~cm}$ yields 66.4 events $/ 108 \mathrm{pb}^{-1}$ in the Monte Carlo data sample of $\eta$ 's originating from the IP. Applying the same cut to the final data sample yields 81 events with an invariant mass in the $\eta$ signal region, or an excess of 14.6 events.

To extract an estimate of the total number of beam-gas events in the $\eta$ signal region, $\eta$ events were generated with a flat distribution in $z$ and passed through the detector simulation and all of the event selection routines. The cuts are biased toward events from the origin, so that the resulting $\mathrm{z}$ distribution was no longer flat but broadly peaked around the origin. In Fig. 6.8 the $\mathrm{z}$ distribution for these events is compared to that from Monte Carlo $\eta$ events originating at the origin. The ratio of events with large $z$, as defined above, to all events in the sample generated flat in $\mathrm{z}$, gives a normalization factor of $5.31 \pm .8$. Thus the estimated number of beam-gas events in the $\eta$ signal region is $77.5 \pm 47$, where the error is statistical.

This estimate has been checked by numerically calculating the number of beamgas events. This is given by:

$$
N_{B G}=n_{e^{ \pm}} \times \rho \times \Delta Z \times \sigma(e p \rightarrow e \eta) \times \epsilon .
$$




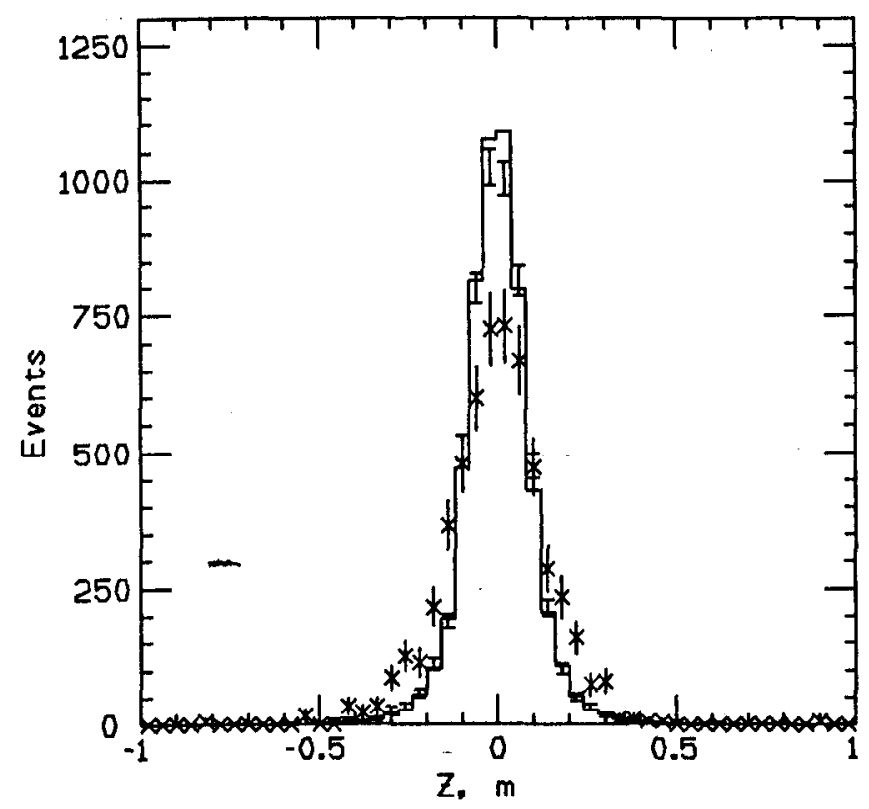

Figure 6.8. Measured $\mathrm{Z}$ distribution of Monte Carlo $\eta$ events originating from the origin (histogram), of data events in the $\eta$ mass region (error bars), and of Monte Carlo $\eta$ events generated flat in $\hat{z}$ (crosses). The distributions are all normalized to the same number of events.

In this expression $n_{e} \pm$ is the number of electrons and positrons which have passed through the IP during data taking, $\rho$ is the density of gas molecules, $\Delta Z$ is the length over which interactions occurred, $\sigma$ is the cross section for electro-production of an $\eta$, and $\epsilon$ is the efficiency for detecting these events.

The number of electrons and positrons passing through the IP during data taking was obtained by multiplying the average beam current for each run by the number of beam crossings during the run. This information was recorded on tape with the data, and the result is $n_{e^{ \pm}}=1.3 \times 10^{24}$. The average vacuum pressure at the IP was approximately $1 . \times 10^{-9}$ Torr, or $3.2 \times 10^{7}$ particles per $\mathrm{cm}^{3} . \Delta Z$ is taken to be $1 \mathrm{~m}$ because beam-gas interactions more than $50 \mathrm{~cm}$ from the IP had a negligible probability of passing all cuts. The effrciency was calculated by generating Monte Carlo beam-gas events, using the EPA for the photon flux and taking the $\eta$ photoproduction cross section as a function of photon energy from fixed target experiments. (37) The proton was assumed to have a flat momentum distribution inside the nucleon, with a cutoff at $250 \mathrm{MeV}$ and events were generated with a flat distribution in $\hat{z}$. The 
events were then passed through the detector simulation and event selection routines, giving the result $\epsilon=.012$. The total cross section to photo-produce $\eta$ 's, calculated using the Monte Carlo generator just described, was .04 $\mu \mathrm{b}$. This must be multiplied by the average atomic number of the gas in the beampipe, which is $50 \% \mathrm{CO}$ and

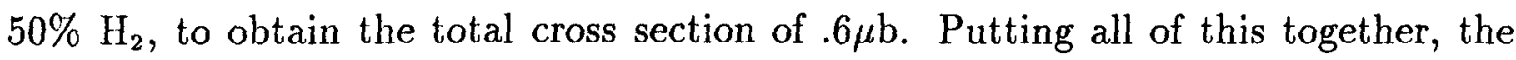
calculation yields 29.1 beam-gas events from the electro-production of $\eta$ 's. Given the uncertainties in estimating many of these quantities, this calculation agrees well with experimental estimate of the number of beam-gas events. An average of the two methods gives an estimated beam-gas background of $53 \pm 47$ events due to photoproduction of $\eta$ 's. The invariant mass distribution of the Monte Carlo beam-gas $\eta$ events normalized to a total of 53 events is shown in fig. 6.9.

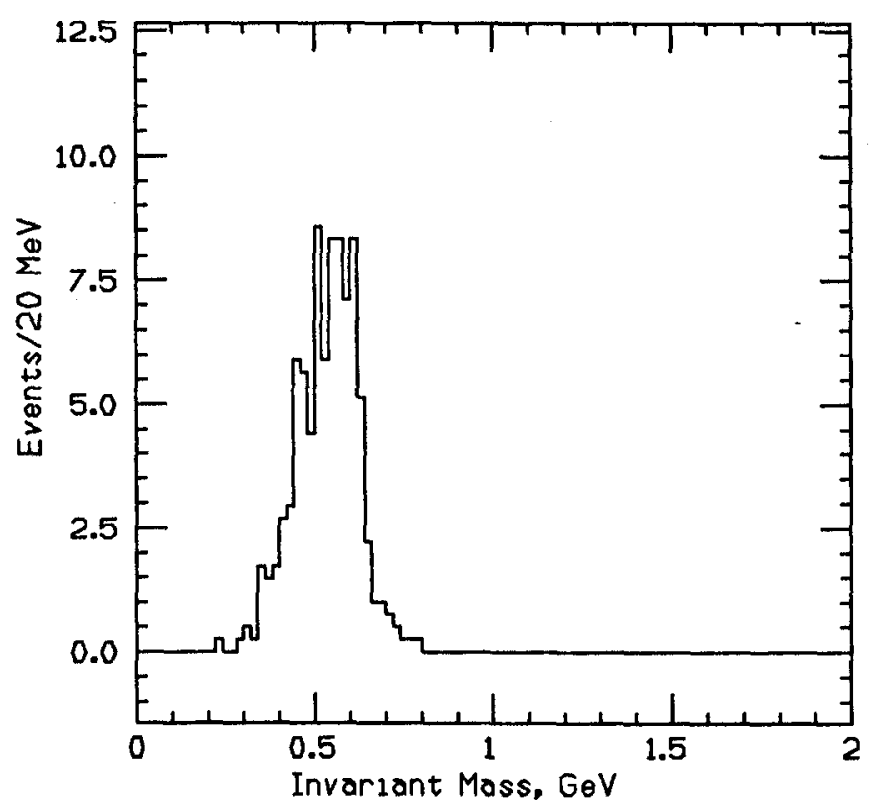

Figure 6.9. Invariant mass distribution of Monte Carlo beam-gas $\eta$ events, normalized to the expected number of beam-gas events in $108 \mathrm{pb}^{-1}$.

\subsection{Summary of Background Contributions}

The backgrounds contributing to the $\eta$ and $\eta^{\prime}$ signal regions are summarized in table 6.1 . The most important background is the $f_{2}(1270)$, which contributes mainly to the $\eta^{\prime}$ region. The number of events from this background is estimated by Monte Carlo simulation as $423 \pm 15$ events, but due to the difficulty of accurately calculating 
the detection efficiency for very low-energy photons $(<100 \mathrm{MeV})$ it is more accurate to fit the data. This will be done in chapter 8 .

Table 6.1. Background contributions to the $\eta$ mass region $(.4-.7 \mathrm{GeV})$ and to the $\eta^{\prime}$ mass region $(.7-1.2 \mathrm{GeV})$.

\begin{tabular}{|c|c|c|}
\hline Process & $\eta$ Region & $\eta^{\prime}$ Region \\
\hline$e^{ \pm} \gamma$ & $2.9 \pm .8$ & $7.8 \pm 1.2$ \\
\hline$f_{2} \rightarrow \bar{\pi}^{\circ} \pi^{\circ}$ & \multicolumn{2}{|c|}{ determined from fit to data } \\
\hline$\gamma \gamma \rightarrow \pi^{\circ} \pi^{\circ}$ continuum & $37 \pm 19$ & $7 \pm 3$ \\
\hline$a_{2} \rightarrow \eta \pi^{\circ}$ & 0 & $6 \pm 1.7$ \\
\hline Beam-gas & $53 \pm 47$ & 0 \\
\hline Total $-f_{2}$ & $92.9 \pm 50.7$ & $13.8 \pm 2.1$ \\
\hline
\end{tabular}

Backgrounds which contribute to $\eta$ region are small; the only significant ones are from continuum two-photon $\pi^{\circ} \pi^{\circ}$ production and from beam-gas production of $\eta^{\prime}$ 's. Unfortunately the systematic errors on both of these backgrounds are large due to the difficulty of estimating them, but fortunately they are small backgrounds. They will be subtracted from the number of $\eta$ events which will be determined by fitting the data in chapter 8 . 


\section{Efficiency Calculations}

The efficiency to detect an event of the type $\eta \rightarrow \gamma \gamma$ or $\eta^{\prime} \rightarrow \gamma \gamma$ is defined as the ratio of events detected to the total which are produced. This definition includes the detector acceptance, the trigger efficiency, and the event selection efficiencies. The efficiencies are calculated sequentially, i.e. the efficiency for a cut is based on the number of events which passed all previous cuts.

The event simulation routines described in chapter 4 have been used extensively in these calculations. Events were generated using the Monte Carlo method described in section 4.1, then passed through the event simulation routines. The resulting Monte Carlo 'data' is then analyzed in exactly the same way as the real data. The fraction of Monte Carlo events which are in the detector acceptance, satisfy the trigger and pass all of the event selection criteria (described in chapter 5) gives the efficiency.

It is important to know not only the efficiency, but also the errors associated with these calculations. The statistical errors are small because the Monte Carlo event samples are large. Almost 400,000 $\eta \rightarrow \gamma \gamma$ and 25,000 $\eta^{\prime} \rightarrow \gamma \gamma$ events were generated, corresponding to a luminosity seven times greater than the data. The systematic errors can be important in some cases. Both the efficiency calculations and the error analysis are presented in this chapter.

The luminosity measurement concludes this chapter. The luminosity, $\mathcal{L}$, together with the observed number of events, $\mathrm{N}$, and the efficiency, $\epsilon$, gives the radiative width 
according:

$$
\Gamma_{\gamma \gamma}^{X}=\frac{\mathrm{N}}{\tilde{\sigma} \cdot \mathcal{L} \cdot \epsilon \cdot \operatorname{Br}(X \rightarrow \gamma \gamma)} .
$$

In this expression, $\tilde{\sigma}$ is the production cross section assuming $\Gamma_{\gamma \gamma}^{X}=1 \mathrm{keV}$, as discussed in chapter 4 , and $\operatorname{Br}(X \rightarrow \gamma \gamma)$ is the branching ratio into two photons, which in the case of the $\eta$ and $\eta^{\prime}$ is known from fixed target experiments.

\subsection{Detector Acceptance}

Both photons from the decay of an $\eta$ or $\eta^{\prime}$ must be within the sensitive part of the lead-glass calorimeter, defined as $20^{\circ}<\theta_{\mathrm{p}}<160^{\circ}$ (for the definition of $\theta_{\mathrm{p}}$ see section 5.2). The detector acceptance is calculated using the Monte Carlo generated samples of $\eta \rightarrow \gamma \gamma$ and $\eta^{\prime} \rightarrow \gamma \gamma$ events, which were passed through the same tracking routines applied to the data. The events were required to have at least two reconstructed leadglass clusters with an energy greater than $100 \mathrm{MeV}$ and $20^{\circ}<\theta_{\mathrm{p}}<160^{\circ}$.

In addition, for the event to be classified as untagged the outgoing electrons had to be willin $21 \mathrm{mr}$ of the beamline. The fraction of untagged events was detcrmined without any detector simulation, by simply calculating the trajectory of the outgoing electrons in each Monte Carlo event. The position of the interaction point, generated with a gaussian distribution about the measured beamspot, was taken into account in this calculation.

The results of the acceptance calculations are summarized in table 7.1. The combined detector acceptance, which includes the calorimeter acceptance and the tagging velo, was $25.9 \%$ for $\eta$ events and $29.4 \%$ for $\eta^{\prime}$ events.

Table 7.1. Detector acceptance for $\eta$ and $\eta^{\prime}$ events.

\begin{tabular}{|l|c|c|}
\hline & $\eta$ & $\eta^{\prime}$ \\
\hline Lead Glass Acceptance & $30.7 \pm 0.6 \%$ & $37.2 \pm 0.6 \%$ \\
\hline No Tag & $84.3 \pm 0.6 \%$ & $82.3 \pm 0.6 \%$ \\
\hline Combined Acceptance & $25.9 \pm 0.5 \%$ & $30.6 \pm 0.5 \%$ \\
\hline
\end{tabular}


The systematic error associated with these calculations depends on how accurately the geometry constants are known. The geometry of the lead-glass calorimeter was determined by an iterative method. For the starting point, the position of each lead-glass block was measured with a ruler. Through-going cosmic rays were then used to calculate residuals for each block, and the constants were adjusted to minimize the residuals. This process was repeated until no further improvement could be obtained; the error was estimated to be no more than a few $\mathrm{mm}$ on the position of each block. These geometry constants were used in the Monte Carlo data simulation.

To check the accuracy of the geometry constants we can compare the $\theta$ distributions of the data with the Monte Carlo, using two data samples. The first is the $\eta$ data, in the invariant mass region from 0.4 to $0.7 \mathrm{GeV}$ where there is little background. This comparison is shown in fig. 7.1; the points are the data and the histogram is the Monte Carlo normalized to the same number of events. Fairly good agreement is observed, but the statistical errors are too large for a precise comparison.

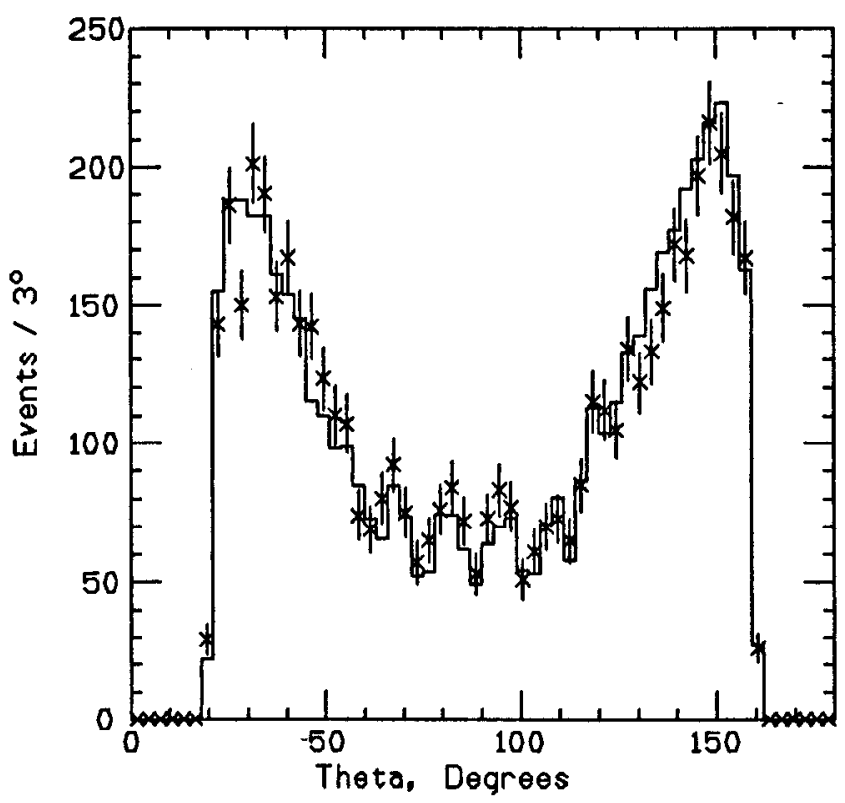

Figure 7.1. $\theta$ distribution of $\eta$ events passing all event selection criteria. The data are the points, the histogram is the Monte Carlo simulation normalized to number of events in the data.

For higher statistics we turn to another data sample, the untagged QED process $e^{+} e^{-} \rightarrow\left(e^{+} e^{-}\right) e^{+} e^{-}$. Because these 'e-pair' events are primarily produced through a 
two-photon like diagram, they are very similar to the $\eta$ and $\eta^{\prime}$ data samples; the final state consists of two lead-glass clusters back-to-back in the azimuthal plane, with a forward-peaked $\theta$ distribution.

Untagged events from the process $e^{+} e^{-} \rightarrow\left(e^{+} e^{-}\right) e^{+} e^{-}$were written out together with $\eta$ and $\eta^{\prime}$ events by the first pass filter. The charged events were then separated from the neutral events by a different set of final event selection criteria. The main difference was that both tracks were required to be charged rather than neutral. Several cuts which were applied to the neutral events were not applied to the e-pairs. The software trigger cut was not applied. Also, because the central tracker provides a $\phi$ measurement for charged tracks, the e-pairs were not required to have a central PWC $\phi$ measurement. The shower shape cuts were not applied to the e-pairs, and the requirement that $\Delta \phi \geq 135^{\circ}$ was also omitted. The stray energy cut was set to $50 \mathrm{MeV}$, rather than the more stringent $25 \mathrm{MeV}$ cut applied to the neutral data. Finally, the acceptance for e-pairs was $30^{\circ} \leq \theta_{\mathrm{p}} \leq 150^{\circ}$ rather than $20^{\circ} \leq \theta_{\mathrm{p}} \leq 160^{\circ}$. (This was simply because of an error in generating the Monte Carlo data sample; due to the large amount of CPU time necessary to generate the Monte Carlo sample, it was more expedient to change the acceptance.) Other than these cuts which were omitted or modified, the selection procedure for e-pairs was identical to that for the $\gamma \gamma$ data sample.

The data sample of e-pairs consisted of approximately 220,000 events; a Monte Carlo sample corresponding to about one-third the luminosity of the data was also generated, using the AXO computer program ${ }^{(38)}$ modified to generate unweighted events. This routine was checked against an exact Monte Carlo event generator and found to be in excellent agreement. ${ }^{(39)}$ The Monte Carlo events were simulaled, tracked and passed through the same event selection routines. Using only the leadglass information to calculate $\theta$ (and ignoring the central tracker information), the $\theta$ distributions for data and Monte Carlo shown in fig. 7.2 were obtained. Excellent agreement is observed.

In both the $\eta$ data and the e-pair data the $\theta$ distributions are not smooth, but 


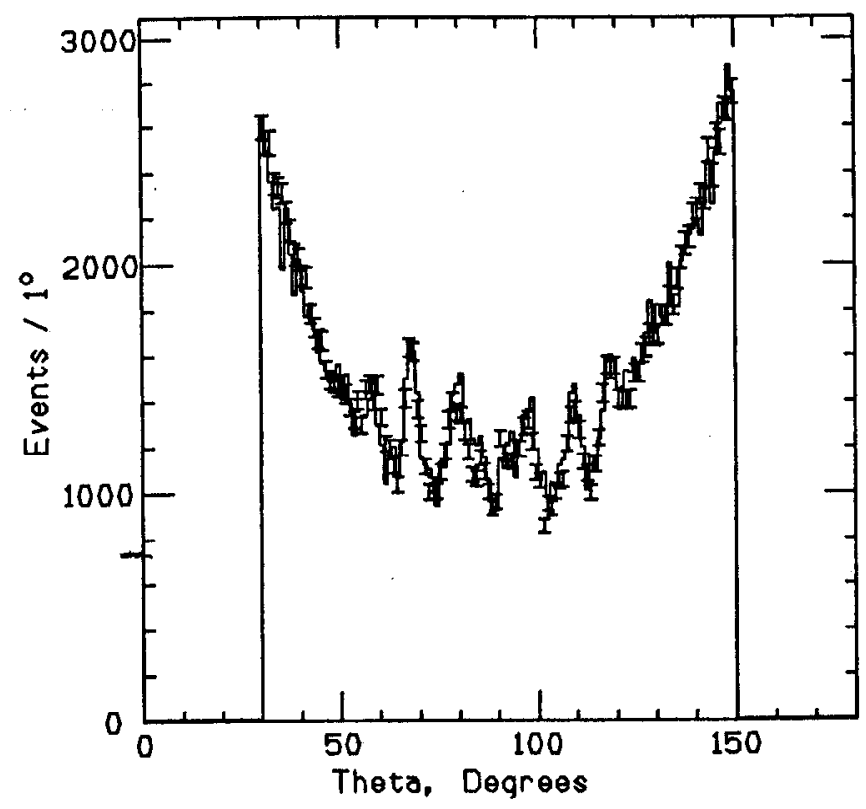

Figure 7.2. $\theta$ distribution of e-pair events. The data are the points, the histogram is the Monte Carlo simulation normalized to number of events in the data.

ragged, reflecting the granularity of the lead-glass calorimeter (each block is $6 \times 6$ $\mathrm{cm}$ ). The good agreement between the data (representing the 'true' geometry) and the Monte Carlo (the calculated geometry) shows that the geometry constants are accurate to better than $1^{\circ}$ at $90^{\circ}$, corresponding to an error in block position of 3.2 $\mathrm{mm}$. The uncertainty in angle at the $20^{\circ}$ cutoff is $\pm .12^{\circ}$, resulting in an uncertainty in the lead-glass acceptance of $0.6 \%$.

The forward tag veto at $21 \mathrm{mr}$ from the beamline was defined by a tungsten mask in the beampipe. Assuming that the position of the veto is accurate to within $1 \mathrm{mr}$ results in an error of $.6 \%$ in the no-tag acceptance.

The combined error is obtained by adding the systematic errors in quadrature, since the lead-glass geometry and the beam-pipe geometry are assumed to have independent errors. The results are given in table 7.1.

\subsection{Trigger Efficiency}

We have previously noted that the lowest threshold of the lead-glass trigger was approximately $.7 \mathrm{GeV}$, so the trigger efficiency for $\eta$ 's $(\mathrm{M}=.549 \mathrm{GeV})$ will obviously not be $100 \%$. For the $\eta^{\prime}(\mathrm{M}=.957 \mathrm{GeV})$ the situation is much better and the trigger 
efficiency is close to $100 \%$.

To calculate the trigger efficiency we rely on the detailed trigger simulation described in section 4.3. The event simulation routines produce the raw signal from each PMT, including the effects of PMT gain and attenuation in the lead glass. The trigger logic is then applied to the raw signals, including threshold effects. The trigger efficiency for $\eta$ events which are in the detector acceptance is $47.9 \%$, and for $\eta^{\prime}$ events it is $95.0 \%$. In order to eliminate the threshold region where the trigger efficiency is changing rapidly, a 'softwa trigger' cut at 2400 counts has been introduced, as discussed in chapter 5. This restricts the acceptance to a region where the trigger efficiency is high and fairly flat. The cfficiency for the combined hardware and software triggers is $40.9 \%$ on $\eta$ events and $94.7 \%$ on $\eta^{\prime}$ events. At the cost of a small loss in efficiency we have eliminated a region which contributes a significant systematic error.

The systematic error on the trigger requirement has several sources. Uncertainty in the energy scale of the Monte Carlo introduces an error in the number of events passing both the software trigger and the hardware trigger. Likewise, uncertainty in the cnergy resolution introduces an error because of smcaring effects. Finally, inaccuracies in the hardware trigger simulation may result in an additional systematic error.

The energy scale and resolution were originally determined using kinematically fit radiative Bhabhas, as described in section 3.2, and the Monte Carlo scale was determined by requiring the simulated energy to agree with the data using the method described in section 4.2. The $\eta$ peak provides an excellent means of measuring both the energy scale and resolution, and provides a check of the agreement between data and Monte Carlo simulation.

A fit to the entire data set and to the $84 / 85$ and $85 / 86$ data cycles individually gives the results shown in table 7.2 . There is a shift of $1.1 \%$ between the first and second cycles, which can be attributed to the fact that the lead-glass calibration constants were determined separately for the two data cycles. (Fits to smaller data 
sets within each cycle are self-consistent.) The fit to the Monte Carlo $\eta$ peak agrees with the fit to the combined data, within errors. The agreement on the mass was achieved without any tuning of the Monte Carlo energy scale; however the width of the Monte Carlo peak was initially too broad, indicating that too much smearing had been introduced in the event simulation. This was modified to give agreement with the data by reducing the added smearing from $8 \%$ to $6.4 \%$. (This suggests that the resolution for $\eta$ events is slightly better than the $\frac{10 \%}{\sqrt{E}}$ quoted in chapter 3 , and is probably closer to $\frac{9 \%}{\sqrt{E}}$. HFrom the results in table 7.2 an error on the relative energy scale of the Monte Carlo of $1 \%$ was assigned. An error on the width of the $\eta$ peak of $5 \%$ was assumed, which translates to a relative error of $5 \%$ in the amount of smearing added to the detector simulation.

Table 7.2. $\eta$ peak and width measurements.

\begin{tabular}{|c|c|c|}
\hline & $\begin{array}{c}\eta \text { Mass } \\
(\mathrm{MeV})\end{array}$ & $\begin{array}{c}\eta \text { Width } \\
(\mathrm{MeV})\end{array}$ \\
\hline 84/85 data & $543 \pm 2$ & $58 \pm 4$ \\
\hline 85/86 data & $548 \pm 2$ & $52 \pm 3$ \\
\hline All data & $545 \pm 1$ & $56 \pm 1$ \\
\hline Monte Carlo & $542 \pm 0.4$ & $53 \pm 0.3$ \\
\hline
\end{tabular}

Applying a $1.0 \%$ shift in the energy scale to Monte Carlo $\eta$ 's produces a $1.2 \%$ shift in the number of $\eta$ events with at least 2400 counts and a lead-glass trigger. The trigger efficiency is less sensitive to the resolution; changing the amount of smearing by $5 \%$ (rrom $6.4 \%$ to $6.7 \%$ ) changes the efficiency by only $.2 \%$. For the $\eta^{\prime}$, the errors due to the uncertainty in energy and resolution are $.9 \%$ and $.2 \%$, respectively.

To determine the accuracy of the trigger simulation, the e-pair sample described above has been used to compare the effect of the trigger on data and Monte Carlo events. The sample of untagged e-pair events was recorded by the same lead-glass energy triggers which logged out the $\eta$ and $\eta^{\prime}$ samples; the fact that they are charged 
does not affect the trigger efficiency.* The trigger simulation was applied to the Monte Carlo e-pair sample; requiring a lead-glass trigger produced the total leadglass energy distribution shown as a histogram in fig. 7.3; the data are represented by error bars. The Monte Carlo data is absolutely normalized to the luminosity of the data in this plot (determined with low-angle Bhabha events; see section 7.6) and the software trigger cut has not been applied. On the log scale, the agreement between data and Monte Carlo in the region 1.5-3.5 GeV is evident. This region is well above the trigger threshold, and carme used to check the low-angle Bhabha normalization; the result agrees to within $1.8 \%$.

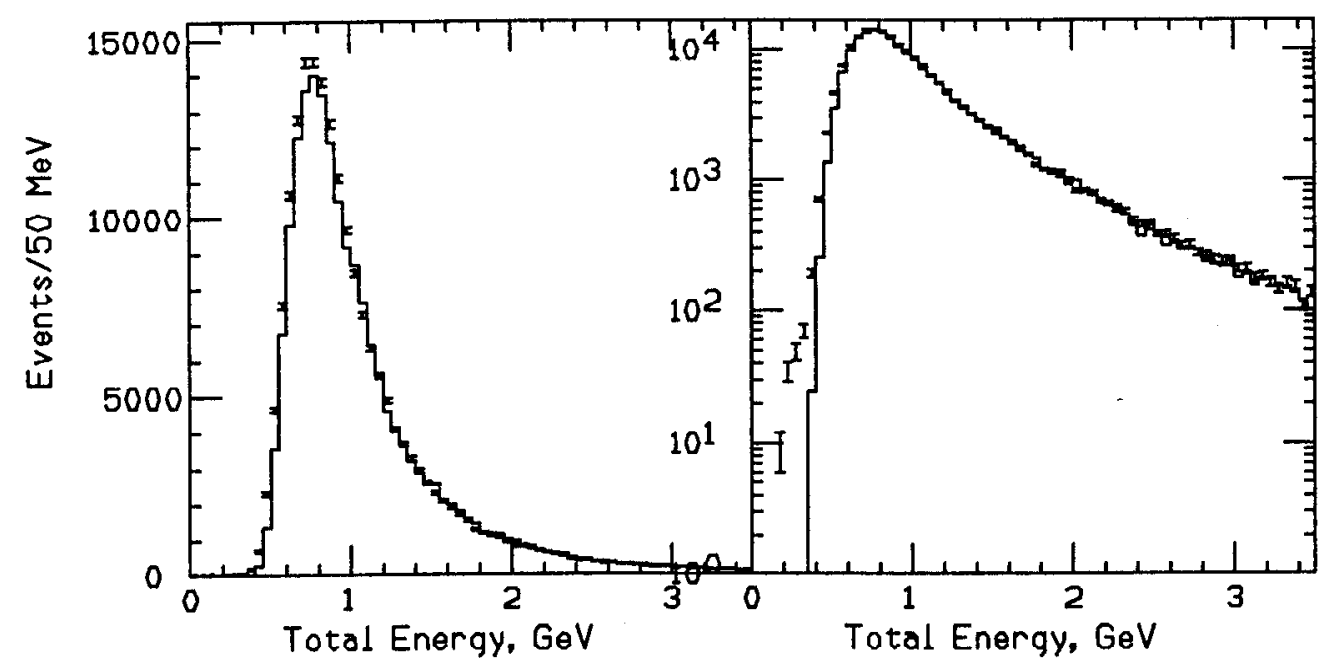

Figure 7.3. Comparison of Monte Carlo and data energy distributions for e-pair events. All events have a lead-glass trigger but the software trigger cut has not been applied.

The rise of events with decreasing energy is due to both the $\frac{1}{s}$ spectrum of the two-photon process and the $\frac{1}{s}$ dependence of the $\gamma \gamma \rightarrow e^{+} e^{-}$cross section. The trigger, by requiring a minimum deposited energy in the lead-glass calorimeter, produces the

* The super-trigger is vetoed if 3 or more of the central VS scintillators fired. The inefficiency due to this veto on e-pairs was determined to be negligible by studying events logged out by a diagnostic trigger, a pre-scaled version of the super-trigger without any vetos. 
observed cut-off in the energy distribution. The comparison between data and Monte Carlo shows that the trigger simulation reproduces the actual trigger response fairly well. The ratio of Monte Carlo events to data gives the ratio of the simulated trigger efficiency to the actual trigger efficiency, bin by bin. From this and the measured trigger efficiency as a function of energy (fig. 2.7), a quantitative estimate of the uncertainty in the number of $\eta$ events due to inaccuracies in the trigger simulation is given by:

$$
-\Delta \mathrm{N}=\sum_{i=1}^{n b i n s}\left(1-\frac{y_{i}}{x_{i}}\right) \epsilon_{i} \mathrm{~N}_{i}
$$

where $\frac{y_{i}}{x_{i}}$ is the ratio of Monte Carlo cvents to data in a given bin, $\epsilon_{i}$ is the trigger efficiency in that bin, and $\mathrm{N}_{i}$ is the number of $\eta$ events per bin before the hardware trigger is applied (but after the software trigger is applied). The result, using a bin size of $100 \mathrm{MeV}$, is an uncertainty of $2.1 \%$ due to inaccuracy in the trigger simulation for $\eta$ events; for $\eta^{\prime}$ events the uncertainty is only .5\%. The quoted uncertainty is a worst case; the effects of changing the energy scale or normalization within their quoted errors is included.

Combining all systematic errors in quadrature yields a trigger efliciency of $40.9 \pm$ $2.43 \%$ for $\eta$ events and $94.7 \pm 1.10 \%$ for $\eta^{\prime}$ events.

\subsection{Production First Pass Efficiency}

The efficiency of the production first pass on $\eta$ and $\eta^{\prime}$ events which were in the detector acceptance and passed the trigger requirement was very high, $98.5 \%$ and 99.4\% respectively. The efficiency of these cuts has been determined by Monte Carlo simulation. This method is very accurate for all but the timing cut. The lead-glass time was required to be within $5 \sigma$ of the beam crossing. No timing information is included in the event simulation, so the efficiency of this cut is estimated using another data set. The radiative Bhabha event sample is suitable because no timing cut was made in selecting these events and there is no background from cosmic rays. The fraction of these events which pass the $5 \sigma$ timing cut is greater than $99.99 \%$; therefore we can neglect any inefficiency from this cut. The individual cuts and efficiencies for 
the production first pass are summarized in table 7.3 ; for a description of the cuts see section 5.1. The systematic errors have been assumed to be negligible.

Table 7.3. Efficiency of production first pass cuts.

\begin{tabular}{|l|c|c|}
\hline Cut & $\eta$ Efficiency & $\eta^{\prime}$ Efficiency \\
\hline Timing & $100.0 \%$ & $100.0 \%$ \\
\hline Anti-Cosmic & $99.7 \%$ & $99.8 \%$ \\
\hline$\leq 80$ C $\overline{\mathrm{T}}$ hits & $99.3 \%$ & $99.6 \%$ \\
\hline$\geq 1$ PbGl Cluster & $99.5 \%$ & $100.0 \%$ \\
\hline Combined & $98.5 \%$ & $99.4 \%$ \\
\hline
\end{tabular}

\subsection{Analysis First Pass Efficiency}

The cuts in the analysis first pass, described in section 5.2 , are highly efficient for signal events. They are also straightforward to determine using the Monte Carlo samples of $\eta$ and $\eta^{\prime}$ events. The results are summarized in table 7.4, which lists each cut and the efficiency for $\eta$ and $\eta^{\prime}$ events.

Table 7.4. Efficiency of analysis first pass cuts.

\begin{tabular}{|c|c|c|}
\hline Cut & $\eta$ Efficiency & $\eta^{\prime}$ Efficiency \\
\hline 2 or 3 PbGl tracks & $99.1 \%$ & $98.9 \%$ \\
\hline 1 track each half & $99.8 \%$ & $99.97 \%$ \\
\hline Inner FS <.15 GeV & $98.3 \%$ & $97.8 \%$ \\
\hline Outer FS <.30 GeV & $99.4 \%$ & $99.4 \%$ \\
\hline Anti-QED & $100.0 \%$ & $100.0 \%$ \\
\hline CPWC Anti-Cosmic & $99.8 \%$ & $99.8 \%$ \\
\hline TOF Anti-Cosmic & $97.2 \pm .3 \%$ & $97.0 \pm .3 \%$ \\
\hline R < 30 cm & $99.6 \%$ & $99.9 \%$ \\
\hline Combined Efficiency & $93.2 \%$ & $92.9 \%$ \\
\hline
\end{tabular}


The only cut which cannot be evaluated using the Monte Carlo data samples is the TOF anti-cosmic cut, because neither the time-of-flight counters nor the leadglass timing was included in the Monte Carlo simulation. The efficiency of this cut has therefore been evaluated using the radiative Bhabha sample. Events with the $e^{+} e^{-} \gamma$ topology were chosen with one electron or photon in the lead-glass acceptance. The probability for the event to fail the TOF cut was determined as a function of the energy and polar angle of the track in the lead-glass calorimeter. This probability was then integrated overthe energy and angular distributions of both tracks in the $\eta$ and $\eta^{\prime}$ Monte Carlo samples. From this analysis the efficiency for the TOF cut was determined to be $97.2 \pm .3 \%$ for $\eta$ events and $97.0 \pm .3 \%$ for $\eta^{\prime}$ events, where the error is due to the statistical uncertainty of the radiative Bhabha sample. The combined efficiency of all cuts in the analysis first pass is $93.2 \%$ for $\eta$ events and $92.9 \%$ for $\eta^{\prime}$ events. The systematic errors have been assumed to be negligible.

\subsection{Final Event Selection Efficiency}

The production and analysis first pass filters have a very high combined efficiency for $\eta$ and $\eta^{\prime}$ events, as the preceding sections have shown. In the final event selection several cuts are made with efficiencies in the $80-90 \%$ range; these cuts are necessary to reject the backgrounds discussed in chapter 6 . The result is a combined efficiency for the final event selection criteria of about $50 \%$ for both the $\eta$ and $\eta^{\prime}$ event samples. The efficiencies of the final analysis cuts as determined by Monte Carlo simulation are displayed in table 7.5 .

The corrections listed in table 7.5 are due to small differences between simulated events and real events. In particular, the efficiencies for both tracks to have a $\phi$ measurement, to be neutral and to pass the stray energy and shower shape cuts depend on the details of the Monte Carlo simulation of the central PWC's, the central tracker and the lead-glass calorimeter. These differences have been estimated using the radiative Bhabha sample, resulting in the correction factors shown in table 7.5. These correction factors, and the associated systematic errors, have been calculated in the manner described below. 
Table 7.5. Efficiency of final event selection cuts. Determination of the correction factors is described in the text. N.A. signifies that a correction factor was not applicable.

\begin{tabular}{|c|c|c|c|c|}
\hline Cut & $\eta$ Efficiency & Correction & $\eta^{\prime}$ Efficiency & Correction \\
\hline Retracked Events & $100.0 \%$ & N.A. & $100.0 \%$ & N.A. \\
\hline 2 PbGl tks, $\theta_{\mathrm{p}}>20^{\circ}$ & $97.5 \%$ & N.A. & $97.2 \%$ & N.A. \\
\hline Both tks have $\phi$ & $83.8 \%$ & $+5.5 \pm 2.9 \%$ & $97.1 \%$ & $+2.9 \pm 2.8 \%$ \\
\hline Both tks neutral & $-84.7 \%$ & $-2.0 \pm 1.0 \%$ & $83.5 \%$ & $-1.6 \pm 1.0 \%$ \\
\hline Not min. ionizing & $98.9 \%$ & N.A. & $99.3 \%$ & N.A. \\
\hline Stray Energy $<25 \mathrm{MeV}$ & $85.0 \%$ & $+1.7 \pm 1.3 \%$ & $83.6 \%$ & $+3.1 \pm 2.2 \%$ \\
\hline Lead Glass Shower Shape & $86.2 \%$ & $-2.6 \pm 3.7 \%$ & $87.8 \%$ & $-5.3 \pm 2.7 \%$ \\
\hline CPWC Shower Shape & $90.2 \%$ & $-0.1 \pm 0.3 \%$ & $90.2 \%$ & $-1.2 \pm 0.8 \%$ \\
\hline$\Delta \phi>135^{\circ}$ & $98.0 \%$ & N.A. & $99.6 \%$ & N.A. \\
\hline Net $\mathrm{p}_{\mathfrak{t}}<.3 \mathrm{GeV}$ & $99.7 \%$ & N.A. & $99.0 \%$ & N.A. \\
\hline Combined & \multicolumn{2}{|c|}{$45.5 \pm 2.6 \%$} & $49.6 \pm 2.8 \%$ \\
\hline
\end{tabular}

To determine how well the simulation reproduces the data, kinematically-fitted radiative Bhabhas with 1 forward track on either side and an electron in the leadglass calorimeter were chosen. The central track was required to be charged so that $\phi$ is well-measured without requiring any signal in the central PWC's. For each event with a satisfactory fit, the 4 -vectors of the particle in the lead-glass calorimeter were written out and simulated with the EGS Monte Carlo. The result is a data sample with a single track in the lead-glass calorimeter and a Monte Carlo sample of single tracks with the same distribution in angle and energy. There are approximately 30,000 events in each sample. The two sets of events are passed through a series of cuts which are similar to the analysis first pass and final event selection criteria, modified for one track instead of two. The efficiency for each cut on data and Monte Carlo is then compared to check for systematic differences. The results are displayed in table 7.6. Very good agreement is obtained for the analysis first pass, justifying the fact that no systematic error was assigned to these highly efficicnt cuts. 
Table 7.6. Efficiency of analysis on data and simulated radiative Bhabhas. N.A. signifies that a cut was not applied to to this sample.

\begin{tabular}{|c|c|c|}
\hline Cut & Efficiency, Data & Efficiency, Monte Carlo \\
\hline \multicolumn{3}{|c|}{ Analysis First Pass } \\
\hline 1 or $2 \mathrm{PbGl}$ tracks & $99.3 \%$ & $98.9 \%$ \\
\hline 1 track each half & N.A. & N.A. \\
\hline Inner $\mathrm{FS}<.15 \mathrm{GeV}$ & $99.1 \%$ & $99.3 \%$ \\
\hline Outer FS $<.30 \mathrm{GeV}$ & N.A. & N.A. \\
\hline Anti-QED & $100.0 \%$ & $100.0 \%$ \\
\hline CPWC Anti-Cosmic & $99.9 \%$ & $99.9 \%$ \\
\hline TOF Anti-Cosmic & N.A. & N.A. \\
\hline $\mathrm{R}<30 \mathrm{~cm}$ & $99.8 \%$ & $99.8 \%$ \\
\hline \multicolumn{3}{|c|}{ Final Event Selection } \\
\hline Retracked Events & $100.0 \%$ & $100.0 \%$ \\
\hline $2 \mathrm{PbGl} \mathrm{tks,}, \theta_{\mathrm{p}}>20^{\circ}$ & $99.4 \%$ & $99.4 \%$ \\
\hline Both tks have $\phi$ & $97.5 \%$ & $96.2 \%$ \\
\hline Both tks neutral & N.A. & N.A. \\
\hline Not min. ionizing & $99.9 \%$ & $99.9 \%$ \\
\hline Stray Energy $<25 \mathrm{MeV}$ & $88.4 \%$ & $86.2 \%$ \\
\hline Lead Glass Shower Shape & $92.9 \%$ & $94.8 \%$ \\
\hline CPWC Shower Shape & $93.7 \%$ & $94.8 \%$ \\
\hline$\Delta \phi>135^{\circ}$ & N.A. & N.A. \\
\hline Net $\mathrm{p}_{\mathrm{t}}<.3 \mathrm{GeV}$ & N.A. & N.A. \\
\hline
\end{tabular}

From this analysis of radiative Bhabhas the efficiency for a track to have central PWC information is obtained as a function of the kinematically fitted track energy. The results for both data and Monte Carlo simulation are shown in fig. 7.4. The efficiency for a track to have a $\phi$ measurement has a strong dependence on the track 
energy, rising to $100 \%$ in $800 \mathrm{MeV}$. The Monte Carlo efficiency is systematically slightly lower. To correct for this, the difference in efficiencies for data and Monte Carlo were folded with the energy and angular distribution of photons from the $\eta$ and $\eta^{\prime}$ samples. For $\eta$ events a correction of $+5.5 \pm 2.9 \%$ was calculated, and for $\eta^{\prime}$ events the correction is $+2.9 \pm 2.8 \%$. The error is due to statistical uncertainty.

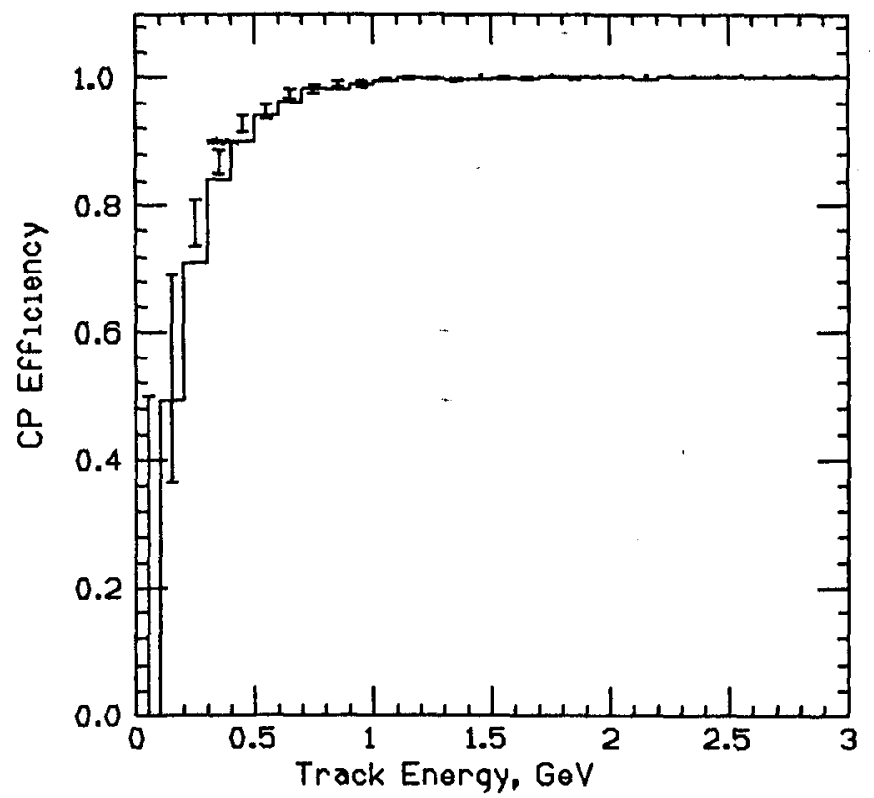

Figure 7.4. Efficiency for a $\phi$ measurement from the central PWC system as a function of track energy. The error bars are the data and the histogram is Monte Carlo simulation.

The next cut, the requirement that both tracks be neutral, was studied using a different method. The probability for a photon to convert in a medium with a depth of $\mathrm{X}$ radiation lengths is given by $1-e^{-7 X / 9}$. At a polar angle of $90^{\circ}$ in the ASP detector, a photon traverses approximately .063 radiation lengths of beampipe and central tracker material before entering the lead-glass calorimeter, so the conversion probability is $4.7 \%$. For events with two photons, integrated over the angular distribution of photons in the $\eta$ Monte Carlo sample, the probability that at least one will convert is about $15 \%$. To check this calculation, which was performed with the EGS Monte Carlo, a comparison of the Monte Carlo with data has been performed using the process $e^{+} e^{-} \rightarrow \gamma \gamma$. Events of this type in which one photon converted in the beampipe or the central tracker were selected from the data, and the number 
was compared to the prediction of QED using the EGS Monte Carlo. ${ }^{(40)}$ This study revealed a small error in the detector simulation: the beampipe thickness had been set to .090 in, when measurement showed that it was in fact .100 in thick.* Also, the corner tubes of the central tracker had been left out of the simulation. Including this extra material and taking into account the background from third-order QED, agreement was found at the $1 \%$ level. The error in the detector simulation has been accounted for by calculating the conversions due to the additional material averaged over the angular distributions of photons in the $\eta$ and $\eta^{\prime}$ samples. The correction factor for the $\eta$ sample is $-2.0 \pm 1.0 \%$ and for the $\eta^{\prime}$ sample it is $-1.6 \pm 1.0 \%$.

The stray energy distributions for data and Monte Carlo in the radiative Bhabha analysis are shown in fig. 7.5. The efficiency is slightly lower for Monte Carlo events, but the shape of the distributions agree very well. Noise in the lead glass is simulated by the random event overlay, and the EGS Monte Carlo should simulate the shower fluctuations which result in extra hits. Although the EGS simulation is very good, it is not perfect, and systematic errors on the order of a few percent may be expected. Assuming that the difference between data and Monte Carlo is due to extra hits associated with real clusters, the disagreement is folded with the energy and angular distribution of photons in the signal. The result is a correction of $+1.7 \pm 1.3 \%$ for the $\eta$ sample and a correction of $+3.1 \pm 2.2 \%$ for the $\eta^{\prime}$ sample. The correction is greater for $\eta^{\prime}$ events becausc the disagrecment is worse at higher energies.

The shower shape cuts have also been studied with radiative Bhabha events. The distribution of the second moment for lead-glass clusters is shown in fig. 7.6 for data and Monte Carlo simulation; in fig. 7.7 the second moment distributions for central PWC clusters is shown. Both the lead glass and central PWC have systematically higher second moments in the data than in the Monte Carlo; this may be attributed to the uncertainty in the geometry constants for the data, which adds an additional term to the calculated moments. As a function of polar angle, the disagreement between data and Monte Carlo on the lead-glass shower shape is more pronounced at wide

* To settle this, the beampipe was actually cut in two with a hack-saw! 


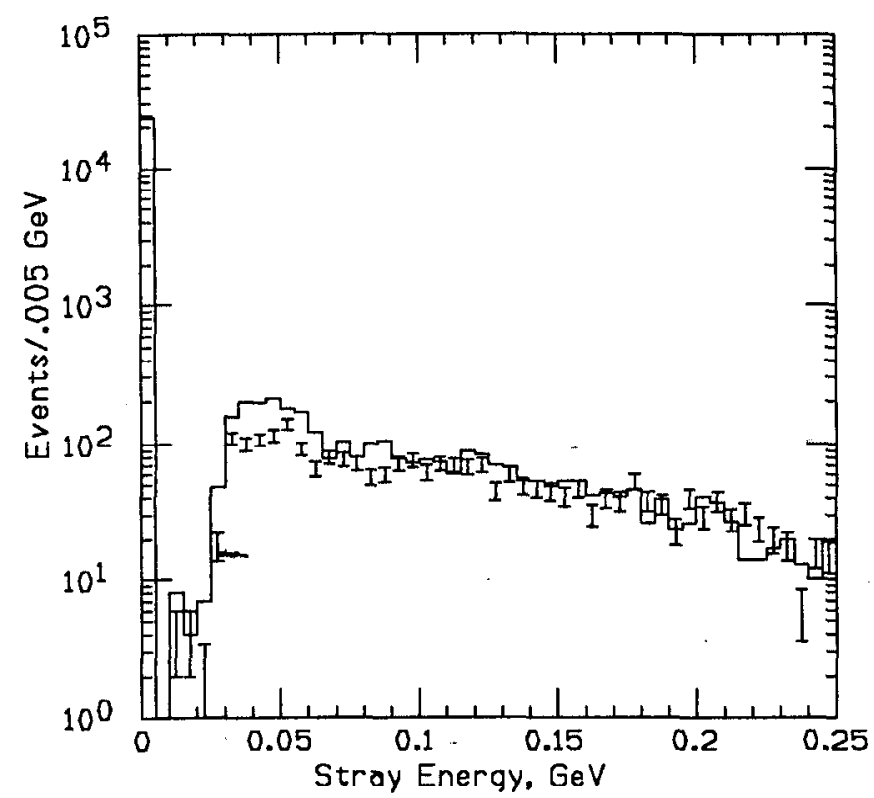

Figure 7.5. Stray energy distribution of Monte Carlo and data measured with radiative Bhabha events; the crosses are data and the histogram is Monte Carlo simulation.

angles, consistent with an uncertainty in the lateral postion of the lead-glass blocks. Averaged over the energy and angular distribution of photons in the $\eta$ sample, the correction for the lead-glass shower shape cut is $-2.6 \pm 3.7 \%$, and for the $\eta^{\prime}$ sample, the correction is $-5.3 \pm 2.7 \%$. The efficiency of the central PWC shower shape cut is almost flat in energy and polar angle; averaged over the $\eta$ sample the correction is $-0.1 \pm .3 \%$, and for the $\eta^{\prime}$ sample the correction is $-1.2 \pm .8 \%$.

Combining all corrections with the calculated efficiencies for the final event selection criteria, the result is an efficiency of $45.5 \pm 2.6 \%$ for the $\eta$ sample and an efficiency of $49.6 \pm 2.8 \%$ for the $\eta^{\prime}$ sample.

In table 7.7 a summary of all efficiencies is presented, together with the combined statistical and systematic errors.

\subsection{Luminosity Measurement}

The integrated luminosity of the data has been determined by measuring the number of low-angle Bhabha events, $e^{+} e^{-} \rightarrow e^{+} e^{-}$, which were detected in the forward calorimeters. ${ }^{(41)}$ The angular region between 60 and $90 \mathrm{mr}$ from the beamline was used; a comparison between the data and the QED Monte Carlo prediction is shown 


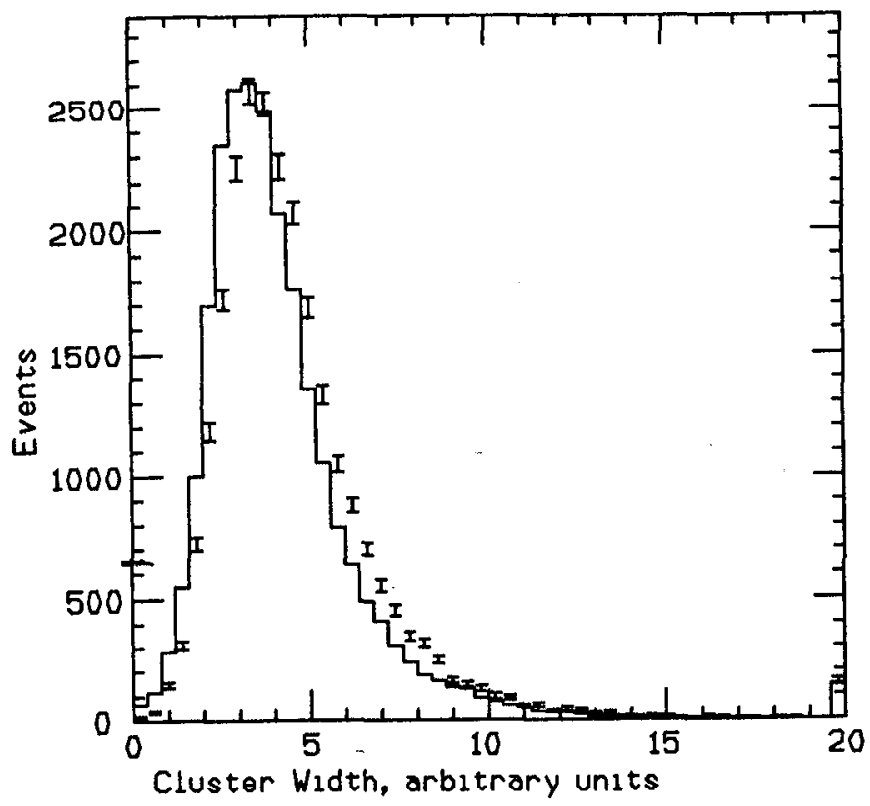

Figure 7.6. Shower shape distributions for lead glass clusters; the error bars are the data and the histogram is Monte Carlo simulation.

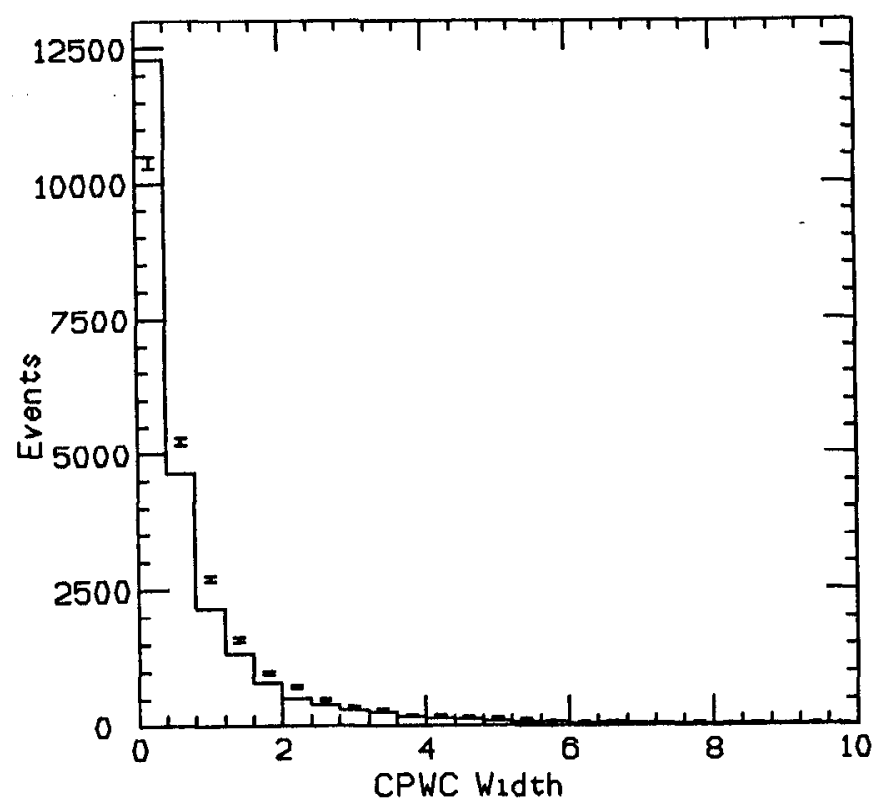

Figure 7.7. Shower shape distributions for central PWC tracks; the error bars are the data and the histogram is Monte Carlo simulation.

in fig. 7.8. The result is a total integrated luminosity of $108.0 \pm .56 \pm .78 \pm .95 \mathrm{pb}^{-1}$ for the data sample used in this analysis, where the first error is statistical, the second error is systematic and the third error is the uncertainty in the QED calculation. 
Table 7.7. Summary of detector acceptance, trigger efficiency and event selection efficiencies. The error on the total efficiency includes both statistical and systematic errors added in quadrature.

\begin{tabular}{|c|c|c|}
\hline & $\eta$ & $\eta^{\prime}$ \\
\hline Detector Acceptance & $25.9 \pm 0.9 \%$ & $30.6 \pm 0.9 \%$ \\
\hline Trigger Efficiency & $40.9 \pm 2.4 \%$ & $94.7 \pm 1.1 \%$ \\
\hline Production First Pass & $98.5 \%$ & $99.4 \%$ \\
\hline Analysis First Pass & $93.2 \pm 0.3 \%$ & $92.9 \pm 0.3 \%$ \\
\hline Final Event Selection & $45.5 \pm 2.6 \%$ & $49.6 \pm 2.8 \%$ \\
\hline Total Efficiency & $4.42 \pm 0.395 \%$ & $13.3 \pm 0.858 \%$ \\
\hline
\end{tabular}

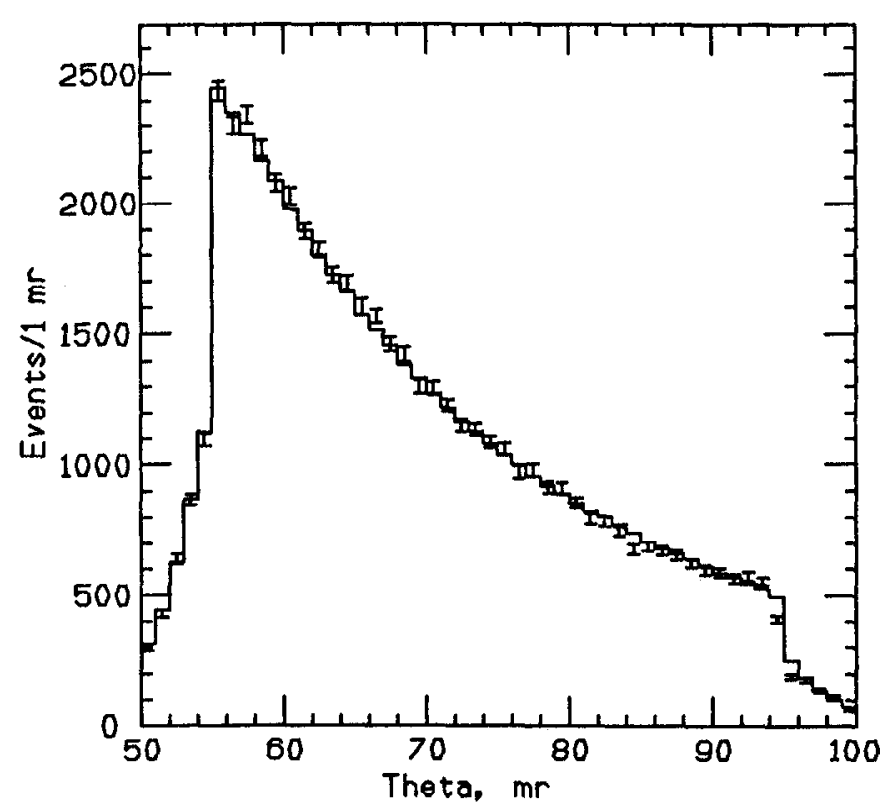

Figure 7.8. Angular distribution of Bhabha events; the crosses are the data and the histogram is the Monte Carlo simulation. 


\section{Results and Conclusions}

\subsection{Fitting the $\gamma \gamma$ Invariant Mass Distribution}

To determine the radiative widths of the $\eta$ and $\eta^{\prime}$ we must extract the number of events of each type in the final data sample. The final $\gamma \gamma$ invariant mass distribution shown in fig. 5.8 has two mass peaks at the $\eta$ and $\eta^{\prime}$ masses and some background, especially above $1 \mathrm{GeV}$. An analysis of background contributions in chapter 6 showed that this is due to two-photon production of the $f_{2}(1270)$, decaying into $\pi^{\circ} \pi^{\circ}$. In addition there is a small amount of background due to other two-photon processes and beam-gas reactions, as summarized in table 6.1. To extract the number of $\eta$ and $\eta^{\prime}$ events, a fit is performed to the sum of the $\eta, \eta^{\prime}$ and $f_{2}$ peaks; the backgrounds listed in table 6.1 are then subtracted from the results of the fit. A binned maximumlikelihood fit method was used, assuming a Poisson distribution for the events in each bin. The program Minuit ${ }^{(42)}$ was used to perform the minimization.

The appropriate form to fit each peak was determined by fitting the Monte Carlo distributions. In fig. 8.1, fig. 8.2, and fig. 8.3 the Monte Carlo invariant mass distributions for the $\eta, \eta^{\prime}$ and $f_{2}$ are shown together with the fits. A Gaussian with power-law tails on both sides was used to describe the $\eta$ peak; because its intrinsic width is much smaller than the resolution of the detector, a Breit-Wigner is not 
necessary. The function used is given by:

$$
\begin{array}{lll}
\frac{A_{1}}{\left(B_{1}-x\right)^{\alpha_{1}}} & \text { for } & x<M-\beta_{1} \sigma \\
e^{-\frac{(x-M)^{2}}{2 \sigma^{2}}} & \text { for } & M-\beta_{1} \sigma<x<M+\beta_{2} \sigma \\
\frac{A_{2}}{\left(B_{2}+x\right)^{\alpha_{2}}} & \text { for } & x>M+\beta_{2} \sigma .
\end{array}
$$

In this expression, $\mathrm{M}$ is the fitted mass, $\sigma$ is the width, and $\alpha_{1}, \beta_{1}, \alpha_{2}, \beta_{2}$ are the parameters describing the lower and upper power-law tails, respectively. The constants $A_{1}, B_{1}, A_{2}, B_{2}$ are determined by the requirement that the function and its first derivatives be continuous.

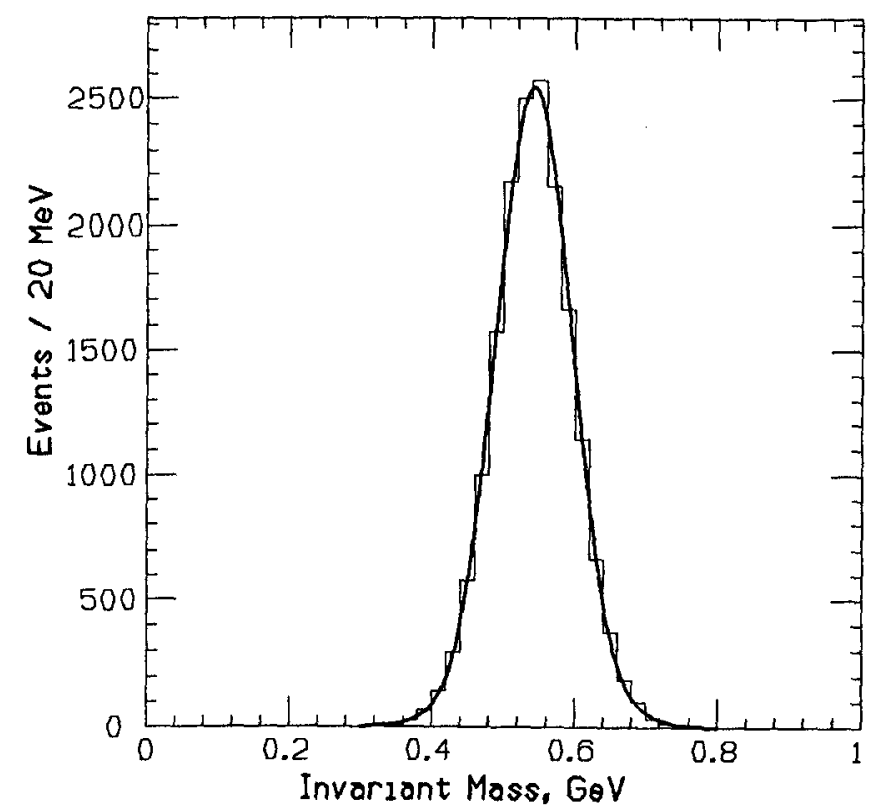

Figure 8.1. Invariant mass distribution of Monte Carlo $\eta$ events passing all cuts.

For the $\eta^{\prime}$ a Gaussian with a single power-law tail on the low side was used. The tail on the low side is rather prominent, and is due to the small fraction of events in which a large amount of energy was lost to pre-radiation or leakage. (The low-side tail is less prominent in the $\eta$ peak because such events would be less likely to pass the trigger; this may also explain why a more complicated function was required to fit the $\eta$ peak.) The $f_{2}$ was described by a Breit-Wigner with a total width of 176 


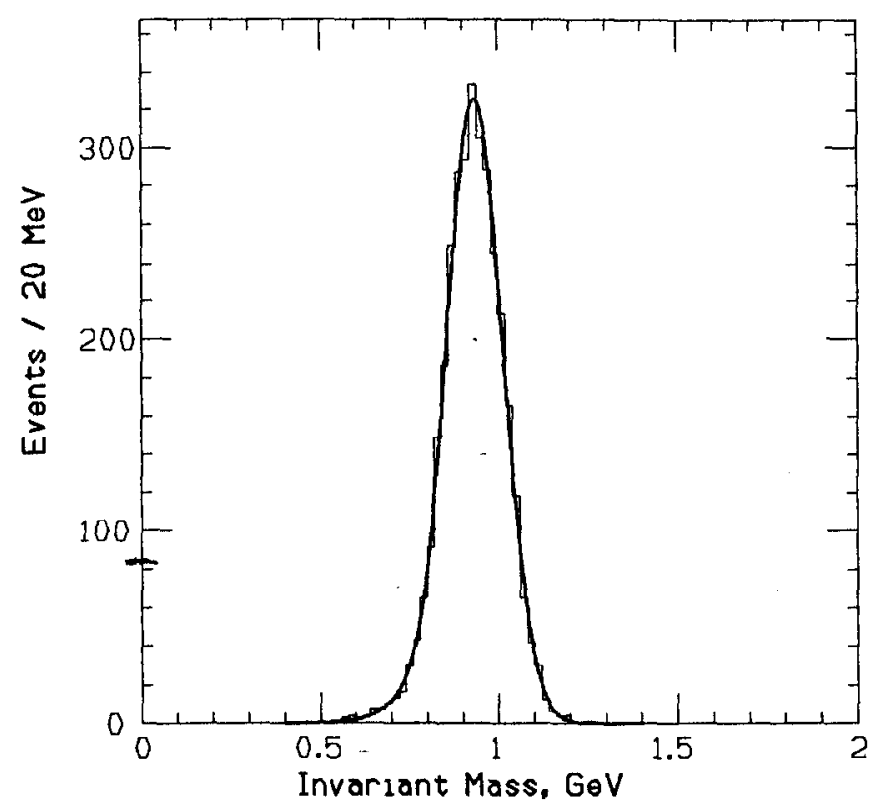

Figure 8.2. Invariant mass distribution of Monte Carlo $\eta^{\prime}$ events passing all cuts.

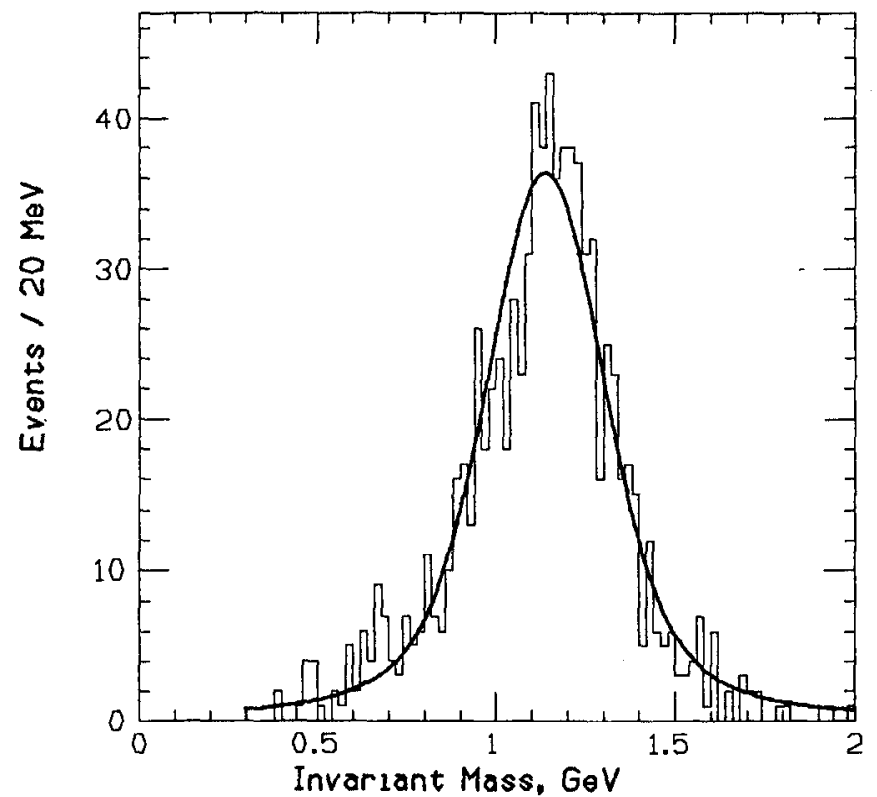

Figure 8.3. Invariant mass distribution of Monte Carlo $f_{2}$ events passing all cuts.

$\mathrm{MeV}$, the value quoted by the 1986 Particle Data Group. The fit results are given in table 8.1 .

In the fit to the data, the number of events in each peak was a free parameter, as were the mean and width of the $\eta$ peak. Because the parameters of the $\eta^{\prime}$ and $f_{2}$ peaks were strongly correlated in the fit, it was necessary to fix the mean and width to 
Table 8.1. Fit parameters for Monte Carlo data samples.

\begin{tabular}{|c|c|c|c|}
\hline & $\eta$ & $\eta^{\prime}$ & $f_{2}$ \\
\hline Mean $(\mathrm{MeV})$ & $542 \pm .4$ & $933 \pm 1.4$ & $1139 \pm 8.0$ \\
\hline Width $(\mathrm{MeV})$ & $54 \pm .4$ & $79 \pm 0.7$ & $125 \pm 9.0$ \\
\hline Resonance Width $(\mathrm{MeV})$ & 0 & 0 & 176 (fixed) \\
\hline$\alpha$ & $20.7 \pm 17.1,1000 \pm 500$ & $15.7 \pm 11.5$ & N.A. \\
\hline$\beta$ & $1.14 \pm .09,1.90 \pm .09$ & $1.61 \pm .10$ & N.A. \\
\hline$\chi^{2}$ Probability & $36 \%$ & $98 \%$ & $4 \%$ \\
\hline
\end{tabular}

agree with the Monte Carlo fits. (To take into account small disagreements between Monte Carlo and data, the ratio of the fitted $\eta$ mass and width to the Monte Carlo values was used to adjust the mass and width of the $\eta^{\prime}$ and $f_{2}$.) The parameters of the power-law tails for the $\eta$ and $\eta^{\prime}$ were also fixed according to the results of the Monte Carlo fits. The systematic errors were determined by changing the values of the fixed parameters, within errors. A good fit to the data is obtained, with a $\chi^{2}$ probability of $47 \%$. The fit is shown in fig. 8.4 , and the results of the fit are given in table 8.2. The number of events in the $\eta$ peak is very stable against changes in the fixed parameters, while the number of events in the $\eta^{\prime}$ peak is sensitive to the details of the fit, resulting in a larger systematic error.

\subsection{The Radiative Widths of the $\eta$ and $\eta^{\prime}$.}

We now have all the information necessary to calculate $\Gamma_{\gamma \gamma}$ according to the prescription of eq. 7.1 :

$$
\Gamma_{\gamma \gamma}=\frac{\mathrm{N}}{\tilde{\sigma} \cdot \mathcal{L} \cdot \epsilon \cdot \operatorname{Br}(x \rightarrow \gamma \gamma)} .
$$

The production cross sections, $\tilde{\sigma}$, are given in section 4.1. The efficiency, $\epsilon$, was discussed in chapter 7 , as was $\mathcal{L}$, the luminosity measurement. The branching ratios of the $\eta$ and $\eta^{\prime}$ to $\gamma \gamma$ are given in table 1.4, and the backgrounds to be subtracted are summarized in table 6.1. All of the quantities necessary to compute the radiative width, including the number of $\eta$ and $\eta^{\prime}$ events from the fit are summarized in table 8.3 


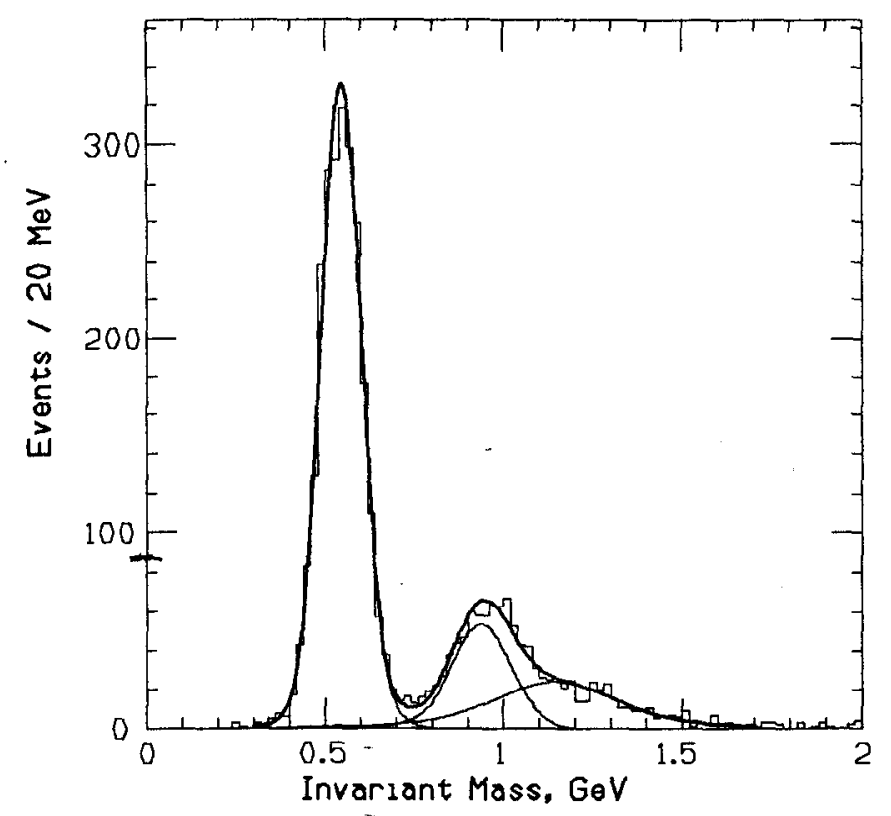

Figure 8.4. Invariant mass distribution of all $\gamma \gamma$ events in the data passing all cuts. The fit is described in the text.

Table 8.2. Fit parameters for $\gamma \gamma$ data. Quantities without errors assigned were fixed in the fit. The probability of the $\chi^{2}$ was $47 \%$.

\begin{tabular}{|c|c|c|c|}
\hline & $\eta$ & $\eta^{\prime}$ & $f_{2}$ \\
\hline Mean $(\mathrm{MeV})$ & $545 \pm 1.3$ & 938 & 1145 \\
\hline Width $(\mathrm{MeV})$ & $57 \pm 1.2$ & 83 & 132 \\
\hline Events & $2380 \pm 49 \pm 25$ & $568 \pm 26 \pm 56$ & $573 \pm 24 \pm 67$ \\
\hline
\end{tabular}

together with the results for the radiative widths. ${ }^{*}$ The results are:

$$
\begin{aligned}
\Gamma_{\gamma \gamma}(\eta) & =.481 \pm .010 \pm .046 \mathrm{keV} \\
\Gamma_{\gamma \gamma}\left(\eta^{\prime}\right) & =4.71 \pm .22 \pm .70 \mathrm{keV}
\end{aligned}
$$

* $\operatorname{Br}\left(\eta^{\prime} \rightarrow \gamma \gamma\right)$ has been the subject of some controversy recently. For many years the average value was $1.87 \pm 0.16 \%$; however a recent measurement by the GAMS experiment ${ }^{(43)}$ has moved the average up to $2.23 \pm 0.18 \%$, a $1.5 \sigma$ shift. The assigned error includes a scale factor of 1.8 , as prescribed by the Particle Data Group, to reflect the inconsistency of these measurements. 
From the radiative width and the branching ratio to $\gamma \gamma$ we can also compute the total width. This calculation yields:

$$
\begin{aligned}
\Gamma(\eta) & =1.24 \pm .012 \mathrm{keV} \\
\Gamma\left(\eta^{\prime}\right) & =211 \pm 37 \mathrm{keV} .
\end{aligned}
$$

The result for the $\eta^{\prime}$ agrees with the direct measurement, $\Gamma\left(\eta^{\prime}\right)=280 \pm 100 \mathrm{keV} .^{(44)}$ The total width of the $\eta$ has been measured directly.

Table 8.3. Summary of the quantities necessary for the calculation of the radiative width of the $\eta$ and $\eta^{\prime}$.

\begin{tabular}{|c|c|c|}
\hline & $\eta$ & $\eta^{\prime}$ \\
\hline$\tilde{\sigma}, \mathrm{nb} / \mathrm{KeV}$ & $2.560 \pm .051$ & $.362 \pm .007$ \\
\hline Efficiency & $.0442 \pm .00395$ & $.133 \pm .00858$ \\
\hline$\gamma \gamma$ branching ratio & $.389 \pm .004$ & $.0223 \pm .0018$ \\
\hline Luminosity, $\mathrm{pb}^{-1}$ & $108.0 \pm 1.4$ & $108.0 \pm 1.4$ \\
\hline Events from fit & $2380 \pm 49 \pm 25$ & $568 \pm 24 \pm 56$ \\
\hline Background & $93 \pm 51$ & $21 \pm 4$ \\
\hline Events - Background & $2287 \pm 49 \pm 57$ & $547 \pm 24 \pm 56$ \\
\hline$\Gamma_{\gamma \gamma}, \mathrm{keV}$ & $.481 \pm .010 \pm .046$ & $4.71 \pm .22 \pm .70$ \\
\hline
\end{tabular}

It is useful to compare these results with other measurements of these quantities. In table 8.4 a summary of all the experimental measurements to date of the $\eta$ radiative width is given. ${ }^{(6)(12)(45-48)}$ The two-photon results arc all in good agreement with one another, but they are in disagreement with the earlier Primakoff measurements. The Primakoff measurements also appear to disagree with one another; however a later analysis of the Bemporad et al. data showed that it was consistent with the value quoted by Browman et al., so the latter measurement supersedes the former. The average of the two-photon measurements is $.511 \pm .026 \mathrm{keV}$, in serious disagreement 
Table 8.4. Summary of all experimental determinations of the radiative width of the $\eta$.

\begin{tabular}{|c|c|c|}
\hline Experiment & Technique & $\Gamma_{\gamma \gamma}(\eta), k c V$ \\
\hline Bemporad, et. al., 1967 & Primakoff & $1.00 \pm .22$ \\
\hline Browman, et. al., 1974 & Primakoff & $0.324 \pm .046$ \\
\hline Crystal Ball at SPEAR, 1984 & $\gamma \gamma \rightarrow \eta \rightarrow \gamma \gamma$ & $0.56 \pm .12 \pm .10$ \\
\hline JADE, 1985 & $\gamma \gamma \rightarrow \eta \rightarrow \gamma \gamma$ & $0.53 \pm .04 \pm .04$ \\
\hline TPC/2 $\bar{\gamma}, 1986$ & $\gamma \gamma \rightarrow \eta \rightarrow \gamma \gamma$ & $0.64 \pm .14 \pm .13$ \\
\hline Crystal Ball at DORIS, 1987 & $\gamma \gamma \rightarrow \eta \rightarrow \gamma \gamma$ & $0.514 \pm .017 \pm .035$ \\
\hline ASP, 1988 & $\gamma \gamma \rightarrow \eta \rightarrow \gamma \gamma$ & $0.481 \pm .010 \pm .046$ \\
\hline
\end{tabular}

with the result of Browman et al.. The inconsistency may be due to an underestimate of the systematic errors in the Primakoff experiments. ${ }^{(49)}$

There are many more results available for the radiative width of the $\eta^{\prime}$; it was in fact the first resonance observed in $\gamma \gamma$ collisions ${ }^{(50)}$ and has been a popular object of study ever since. In table $8.5,{ }^{*}$ the measured values of the radiative width of the $\eta^{\prime}$ are summarized. ${ }^{(12)(51-60)}$ A number of different decay modes of the $\eta^{\prime}$ have been used; each suffers from its own problems. In the case of the $\rho \gamma$ decay mode, earlier measurements (JADE, CELLO) did not use the correct M1 decay matrix element. A more general problem is that the energy of the photon at the nominal $\rho$ mass is only $170 \mathrm{MeV}$ for the decay of an $\eta^{\prime}$ at rest in the lab frame. For many detectors, the efficiency to detect such low energy photons is not very high and the result is sensitive to the photon energy spectrum and the details of the photon detection efficiency . The $\eta \pi \pi$ mode also requires good photon detection at low energies. The $\gamma \gamma$ final state would appear to avoid many of these difficulties; however the small branching ratio

* In cases where different branching ratios have been used the quoted experimental values have been changed from the published values for consistency. The branching ratios used are: $\operatorname{Br}\left(\eta^{\prime} \rightarrow \rho \gamma\right)=.300 \pm .016, \operatorname{Br}\left(\eta^{\prime} \rightarrow \eta \pi \pi\right)=.652 \pm .016, \operatorname{Br}\left(\eta^{\prime} \rightarrow\right.$ $\gamma \gamma)=.0223 \pm .0018$. 
to $\gamma \gamma$ relative to the other modes is a serious disadvantage. The recent controversy in the value of $\operatorname{Br}\left(\eta^{\prime} \rightarrow \gamma \gamma\right)$ adds an additional uncertainty. Given these experimental difficulties, the agreement which is obtained between the averages for the various modes is rather remarkable.

Table 8.5. Summary of all experimental determinations of the radiative width of the $\eta^{\prime}$.

\begin{tabular}{|c|c|c|}
\hline Experiment & Decay Mode & $\Gamma_{\gamma \gamma}(\eta), k e V$ \\
\hline JADE, 1982 & $\rho \gamma$ & $5.0 \pm 0.5 \pm 0.9$ \\
Mark II, 1983 & $\rho \gamma$ & $5.8 \pm 1.1 \pm 1.2$ \\
CELLO, 1983 & $\rho \gamma$ & $6.2 \pm 1.1 \pm 0.8$ \\
PLUTO, 1984 & $\rho \gamma$ & $3.80 \pm 0.26 \pm 0.43$ \\
TASSO, 1984 & $\rho \gamma$ & $5.1 \pm 0.4 \pm 0.7$ \\
TPC/2 $\gamma, 1987$ & $\rho \gamma$ & $4.5 \pm 0.3 \pm 0.7$ \\
ARGUS, 1987 & $\rho \gamma$ & $3.8 \pm 0.1 \pm 0.5$ \\
\hline Average & $\rho \gamma$ & $4.29 \pm 0.28$ \\
\hline Mark II, 1985 & $\eta \pi^{+} \pi^{-}$ & $4.7 \pm 0.6 \pm 0.9$ \\
Crystal Ball, 1987 & $\eta \pi^{\circ} \pi^{\circ}$ & $4.6 \pm 0.4 \pm 0.6$ \\
\hline Average & $\eta \pi \pi$ & $4.63 \pm 0.60$ \\
\hline JADE, 1985 & $\gamma \gamma$ & $3.3 \pm 0.8$ \\
\hline Crystal Ball,1987 & $\gamma \gamma$ & $4.7 \pm 0.5 \pm 0.5$ \\
\hline ASP,1988 & $\gamma \gamma$ & $4.71 \pm 0.22 \pm 0.70$ \\
\hline Average & $\gamma \gamma$ & $4.30 \pm 0.43$ \\
\hline$\Lambda$ verage & All Modes & $4.33 \pm 0.22$ \\
\hline
\end{tabular}

\subsection{The Pseudoscalar Mixing Angle}

As discussed in section 1.5 , the pseudoscalar mixing angle is related to the $\eta$ and 
$\eta^{\prime}$ radiative widths by:

$$
\begin{aligned}
& \Gamma_{\gamma \gamma}^{\eta}=\frac{\alpha^{2}}{64 \pi^{3}} \frac{M_{\eta}^{3}}{3}\left[\frac{1}{f_{8}} \cos \theta_{p}-\frac{\sqrt{8}}{f_{1}} \sin \theta_{p}\right]^{2} \\
& \Gamma_{\gamma \gamma}^{\eta^{\prime}}=\frac{\alpha^{2}}{64 \pi^{3}} \frac{M_{\eta^{\prime}}^{3}}{3}\left[\frac{1}{f_{8}} \sin \theta_{p}+\frac{\sqrt{8}}{f_{1}} \cos \theta_{p}\right]^{2}
\end{aligned}
$$

This is two equations in three unknowns: $\theta_{p}$, the pseudoscalar mixing angle, $f_{8}$ and $f_{1}$, the decay constants for the $\mathrm{SU}(3)$ basis states $\eta_{8}$ and $\eta_{1}$. The latter cannot be directly measured, so we must make some assumptions. The usual one is that of octet symmetry, in which $f_{8}=f_{\pi}$ because they belong in the same $\mathrm{SU}(3)$ octet. However, symmetry breaking effects turn out to be rather large in this case; a calculation by Donoghue, Holstein and Lin incorporating these corrections ${ }^{(61)}$ yielded the result:

$$
f_{8}=1.25 f_{\pi}
$$

A similar calculation by Gasser and Leutwyler ${ }^{(62)}$ gave a consistent result, $f_{8}=$ $1.21 f_{\pi}$. (Gilman and Kauffman ${ }^{(63)}$ estimate the uncertainty on these calculations to be on the order of $5 \%$.) Using eq. 8.1 to define $f_{8}$ in terms of the measured value for the pion decay constant, $f_{\pi}=93 \mathrm{MeV}$, the radiative widths of the $\eta$ and the $\eta^{\prime}$ as measured in this experiment give the following results:

$$
\begin{aligned}
\theta_{p} & =-19.8^{\circ} \pm 2.5^{\circ} \\
f_{1} & =1.04 \pm .11 f_{\pi}=96 \pm 11 \mathrm{MeV}
\end{aligned}
$$

(Because the equations are quadratic in $\theta_{p}$, there are two possible solutions; the ambiguity is resolved by independent measurements of $\theta_{p}$, for example in $\mathrm{J} / \psi$ decays.) The errors include the $5 \%$ uncertainty on $f_{1}$.

Using this value of the pseudoscalar mixing angle in equation 1.25 gives the quark content of the $\eta$ and $\eta^{\prime}$ mesons:

$$
\begin{aligned}
|\eta\rangle & =.58|\mathrm{u} \overline{\mathrm{u}}+\mathrm{d} \overline{\mathrm{d}}\rangle-.57|\mathrm{~s} \overline{\mathrm{s}}\rangle \\
\left|\eta^{\prime}\right\rangle & =.40|\mathrm{u} \overline{\mathrm{u}}+\mathrm{d} \overline{\mathrm{d}}\rangle+.82|\mathrm{~s} \overline{\mathrm{s}}\rangle
\end{aligned}
$$


According to this result, both the $\eta$ and $\eta^{\prime}$ have significant admixtures of $s \bar{s}$; this is not apparent in their decays, as they are both too light to decay into kaons. This situation is far from the case of 'ideal' mixing, as exemplified by the $J^{P C}=1^{--}$ nonet in which the mixing angle is approximately $35^{\circ}$ resulting in a $\rho$ which is mostly $\mathrm{u} \overline{\mathrm{u}}+\mathrm{d} \overline{\mathrm{d}}$, and decays primarily to pions, while the $\phi$ is mostly s $\bar{s}$ and decays primarily to kaons. The tensor nonet is also very close to ideally mixed. The pseudoscalar nonet may be so far from ideally mixed because the process $\mathrm{d} \overline{\mathrm{d}} \leftrightarrow$ gluons $\leftrightarrow$ ss̃ requires only two gluons, as opposed to three for the vector nonet, and $\alpha_{s}$ is larger due to the lighter masses involved. ${ }^{(64)}$

If we try to determine whether other states are also mixing with the $\eta$ or $\eta^{\prime}$, we immediately run into the following problem: the addition of another state requires a new mixing angle, and we must then attempt to solve 2 equations in 4 unknowns. Even assuming a relationship between $f_{8}$ and $f_{\pi}$, as we did above, this leaves us with one extra variable. Because of the lack of knowledge concerning the value of $f_{1}$ it is not possible to determine whether other states, such as charmonium or gluonium, are mixing with the $\eta$ or the $\eta^{\prime}$ from a measurement of the radiative widths alone.

There are other experiments which are sensitive to the pseudoscalar mixing angle, such as measurements of $J / \psi$ decays and of radiative decays of the light vector mesons. The results are compatible with measurements of the radiative widths, and so far there is no evidence for mixing with additional states. ${ }^{(65,66)}$ There is also agreement with the theoretical prediction using the quadratic Gell-Mann Okubo mass formula:

$$
4 M_{K}^{2}=M_{\pi}^{2}+3\left(M_{\eta}^{2} \cos ^{2} \theta_{p}+M_{\eta^{\prime}}^{2} \sin ^{2} \theta_{p}\right)
$$

if one-loop chiral corrections are taken into account. ${ }^{(61)}$ In a comprehensive review of the subject, Gilman and Kauffman concluded that both theoretical predictions and current experimental evidence are consistent with $\theta_{p} \simeq-20^{\circ}$. in the framework of the simple $\mathrm{SU}(3)$ model. ${ }^{(63)}$. 


\subsection{Summary and Conclusions}

Two-photon production of the $\eta$ and $\eta^{\prime}$ mesons has been observed with the ASP detector at the PEP $e^{+} e^{-}$storage ring. Both were detected in the $\gamma \gamma$ decay mode, in an untagged configuration. The radiative widths of the $\eta$ and $\eta^{\prime}$ were determined to be:

$$
\begin{aligned}
\Gamma_{\gamma \gamma}(\eta) & =.481 \pm .010 \pm .046 \mathrm{keV} \\
\Gamma_{\gamma \gamma}\left(\eta^{\prime}\right) & =4.71 \pm .22 \pm .70 \mathrm{keV}
\end{aligned}
$$

in agreement with previous measurements. (One exception to this is that the radiative width of $\eta$ as determined by the Primakoff technique is not in agreement with the value found here.) The statistical accuracy of the measurements exceeds that of any previous two-photon measurements using the $\gamma \gamma$ decay mode, and the combined statistical and systematic errors are comparable to the precision achieved in the most accurate measurements to date. Using the measured radiative widths of the $\eta$ and $\eta^{\prime}$, the pseudoscalar mixing angle is calculated. The result, $\theta_{p}=-19.8 \pm 2.5^{\circ}$, is in agreement with other experimental methods which have been employed. 


\section{Appendix A}

\section{Forward Drift Chambers}

In order to identify charged particles and measure their trajectory in the forward direction, a system of forward drift chambers was designed and built as part of the ASP detector. The forward drift chambers were located symmetrically on either side of the interaction point between the inner and outer forward shower counters. There were two drift chamber modules on each side, at $z= \pm 1.6 \mathrm{~m}$, the 'inner' drift chambers, and at $z= \pm 3.0 \mathrm{~m}$, the 'outer' drift chambers. Each module consisted of two drift chamber planes, one with the wires oriented vertically to measure the $\hat{x}$ coordinate and the other with wires oriented horizontally to measure the $\hat{y}$ coordinate.

The angular acceptance of the outer drift chambers was 20 to $100 \mathrm{mr}$ from the beamline; for the inner drift chambers the acceptance was 50 to $190 \mathrm{mr}$. For charged tracks which were from 50 to $100 \mathrm{mr}$ the drift chambers provided two measurements in $\hat{x}$ and $\hat{y}$ of the track. The forward drift chambers were the only components of the ASP detector which were surveyed, providing a known frame of reference for the geometry of the other detector elements.

In the following sections, the mechanical construction, electronic read-out and gas system of the forward drift chambers will be described, followed by a brief discussion of the performance. 


\section{A.1 Mechanical Construction}

As mentioned above, each of the four drift chamber modules consisted of two wire planes, oriented orthogonally. Each of the eight drift chamber planes was constructed from two halves which were rectangular in shape with a semi-circular cutout. Two of these chambers were mounted on either side of the beampipe to create a square drift chamber plane with a circular cutout to accomodate the beampipe. The radius of the cutout was 3.15 in for the inner drift chamber modules and 2.15 in for the outer drift chamber modules. Apartfrom the difference in the size of the semi-circular cutout, the sixteen individual chambers were identical in construction.

Each chamber had a machined aluminum skeleton consisting of a spine 39.6 in long, and two L-shaped end-pieces 11.89 in wide which were joined to the spine with dowels. The aluminum pieces were all .5 in thick and 1.77 in across. Holes for the feed-throughs were drilled in the L-shaped end-pieces, so that the wires could be strung parallel to the spine. There were two rows of 19 holes on .59 in spacing, for a total of 38 wires in each chamber. The active area of the each chambers was 25.5 by 11.5 in, minus the semi-circular cutout. The read-out electronics were mounted on both ends in the space formed by the $\mathrm{L}$ and the spine; slots in the aluminum were machined so that the circuit boards could be slid in and out.

The chambers were covered on both sides with .063 in thick copper-clad G-10. The G-10 was fastened to the aluminum skeleton with a row of screws on 1 in spacing. An O-ring groove was machined in the aluminum spine and end-pieces inside the row of screws to make the chambers gas tight. In addition, RTV was used on all joints. A center ring of machined G-10 was glued inside the semi-circular cutout in the G-10 plate. The semi-circular ring consisted of three layers, each with slots machined in them to allow the wires to be strung through them. After the wires were strung, Dolph's epoxy was injected into the slots to hold the wires. After it had cured the wires were trimmed out of the semi-circle. The openings on either side of the G-10 ring were closed up by soldering thin copper skins to the G-10 plates and sealing the edges with RTV.

The feedthroughs were machined from Delran and were a snug fit in the machined 
holes. To ensure that the chambers would be gas tight the feedthroughs were epoxied in place. The wires which were strung through the feedthroughs were alternating sense wires, of $30 \mu \mathrm{m}$ gold-plated tungsten, and field wires, of $150 \mu \mathrm{m}$ copper-plated beryllium. The two rows of wires were arranged in an alternating pattern so that the nearest neighbors of each sense wire were field wires. The tension in the wires was set by weighting them with weights strung over a pulley. This tension was chosen to ensure that the wire displacement due to gravitational sag would be insignificant. The displacement, $\delta$, of a wireof length $l$ and weight per length $\omega$, strung at a tension $T$, is given by

$$
\delta=\frac{\omega l^{2}}{8 T}
$$

For the sense wires, a tension of 100 gm ensured that the gravitational sag would be less than half the wire diameter. For the heavier field wires a tension of $300 \mathrm{gm}$ was required.

After the wires were strung they were held in place by inserting a pin into the feedthrough with a drop of epoxy. The pin made electrical contact with the wire, and it had an attached piece of multi-strand electrical wire which was soldered to a circuit board for the electronic read-out. In the case of the field wires, this read-out wire was soldered to a copper ground plate attached to the chamber body.

\section{A.2 Operation and Electronic Read-Out}

The chambers were operated at atmospheric pressure with a gas mixture of $48.2 \%$ Argon, $48.2 \%$ ethane and $1.6 \%$ ethyl alcohol vapor $\left(\mathrm{C}_{2} \mathrm{H}_{5} \mathrm{O}_{4}\right)$. The alcohol vapor was introduced by bubbling the gas through a container of ethyl alcohol (200 proof) at $0^{\circ} \mathrm{C}$. The purpose of the alcohol was to reduce the possibility of wire damage due to the occasional high currents drawn by the chambers, which were located close to the beampipe. Alcohol attacks epoxies which are not fully cured and may also attack RTV if present in quantities greater than a few percent. However no damage due to the alcohol was observed in the forward drift chambers.

The sense wires were operated at $+2.6 \mathrm{kV}$ and the field wires were at ground. The signals from the sense wires were capacitively coupled to a Lecroy HIL440 hybrid 
amplifier/discriminator, which came in a four-channel 24-pin dual in-line package. This hybrid model had an externally adjustable threshold via a control voltage, and required $\pm 5 \mathrm{~V}$ and $-2.5 \mathrm{~V}$ supplies. The output was a differential ECL twisted pair cable, which carried the signal a couple hundred feet away to the electronics building. There the signals were fed into time-to-amplitude modules (TAC's), which produced a signal proportional to the delay between the beam crossing and the observed signal. This information was recorded on magnetic tape.

Each sense wire wastead out on one end only. Sense wires which intersected the circular cut-out were of course read out on each end. For the large radius chambers there were 5 such bisected wires, and there were 3 for the small-radius chambers. Given 19 sense wires in each chamber, the total number of channels to read out was 24 and 22, respectively, for the large and small-radius chambers. The channel count for all sixteen chambers was 368 .

A circuit board was designed with four Lecroy hybrids, providing 16 channels of read-out. However, on half of them only two hybrids were actually mounted; one fully stuffed card and one half-stuffed card were then mounted on each end of the chambers. In order to make it easy to replace a circuit board with dead channels, the cards were designed to slide into Camac-style receptacles which were mounted on another board to which the sense wire leads were soldered.

\section{A.3 Performance}

The drift chambers performed well; there were almost no dead channels and the effects of beam-related noise were negligible due to copper shielding around the electronics and proper signal grounding. The occupancy was higher than expected from single charged tracks on low-angle Bhabha events, however, due to carly showering in the beam-pipe material. The extra hits made it difficult to determine the precise trajectory for such tracks.

For charged tracks which did not begin to shower in the beam-pipe, signals would be registered by two sense wires in each plane through which the track passed. The time of the hit on each wire was proportional to the distance of the track from the 
wire; by comparing the measurements of the two wires, the error of the measurement can be determined. This is shown in fig. A.1; the difference between the positions as measured by two adjacent wires is plotted. The resolution is given by $\frac{\sigma}{\sqrt{2}}$; the result is a resolution of about $300 \mu \mathrm{m}$.

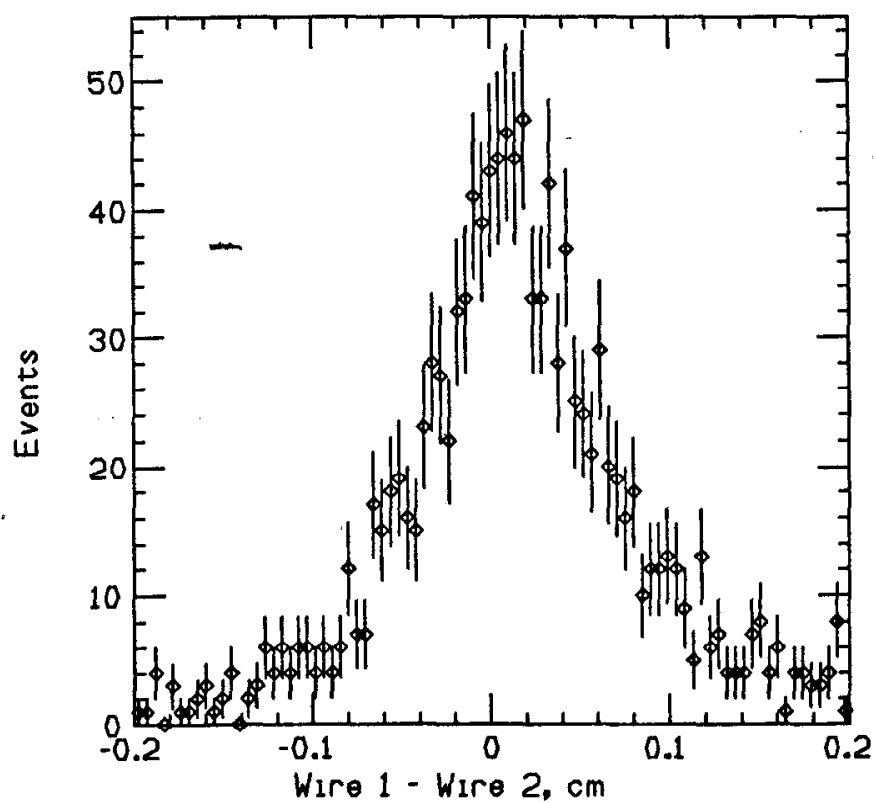

Figure A.1. Difference between measured position of track from two adjacent wires in the drift chambers. The resolution for a single measurement is estimated from this to be about $300 \mu \mathrm{m}$.

The resolution was somewhat worse than expected; this was due to the fact the drift velocity of ionized electrons was not constant over the width of a cell. Before the alcohol vapor was added, the drift velocity had been roughly constant; however the effect of alcohol is to raise the electric field strength at which the drift velocity reaches a plateau. The calculated drift velocity across the cell both with and without alcohol is shown in fig. A.2; the calculation used electrostatic field calculations performed on a computer together with drift velocity vs. electric field measurements performed in studies for the MarkII vertex chamber. ${ }^{(67)}$

A correction for this effect was applied but it was only an approximation, resulting in a somewhat worsened resolution. However due to the long lever arm of the forward drift chambers, the achieved resolution was satisfactory for studies of the beamspot position and determination of geometry constants. 


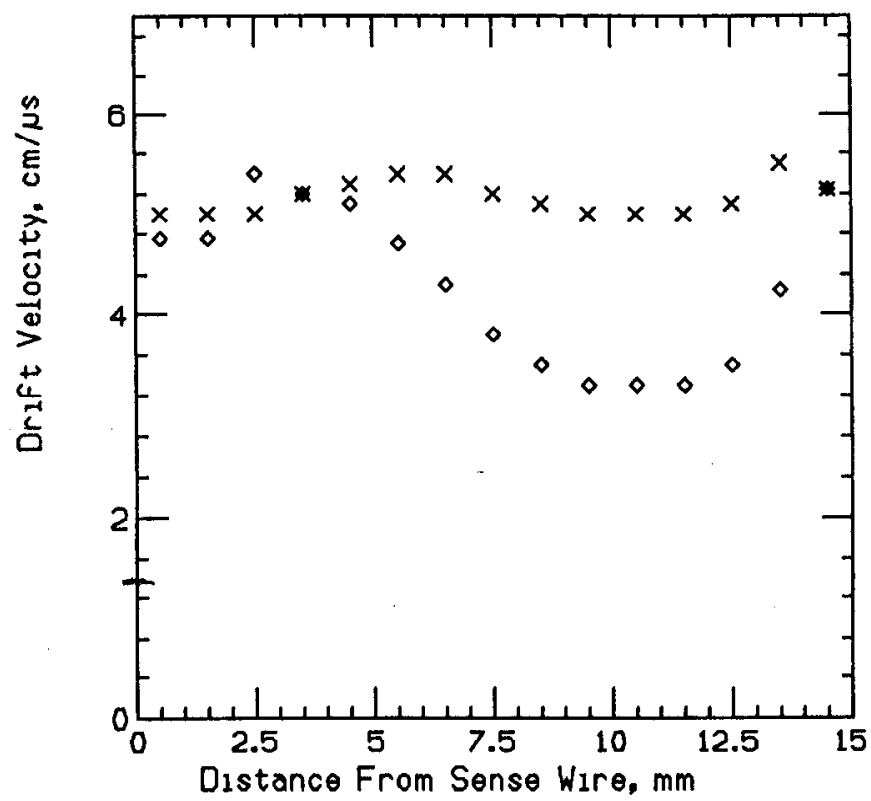

Figure A.2. Calculated drift velocity in forward drift chambers vs distance from sense wire. The X's are for a gas mixture of $50 \%$ argon, $50 \%$ ethane. The O's are the same with the addition of $1.6 \%$ ethyl alcohol vapor.

\section{References}

1. Landau and Lifshitz, Phys. Zs. Soviet Union 6 (1934) 244

2. J. D. Jackson, Classical Electrodynamics, John Wiley and Sons, New York. 1962 p. 10.

3. Euler and Kockel, Naturwissenschaften26 (1935) 246

4. Breit and Wheeler, Phys. Rev. 46 (1934) 1087

5. H. Primakoff, Phys. Rev. 81 (1951) 899

6. C. Bemporad, et al., Phys. Lett. B25 (1967) 280

7. F. E. Low, Phys. Rev. 120 (1960) 582 
8. E. Williams, Phys. Rev. 45 (1934) 729

9. C. F. von Weizsäcker, Jzp88 (1934) 612

10. V. Balakin, et al., Phys. Lett. B34 (1971) 663

11. C. Bacci, et al., Nuovo Cim. 3 (1972) 709

12. D. Williams, et al.(Crystal Ball collaboration), Phys. Rev. D38 (1988) 1365

13. For a summary of the Feynman rules, see J. Bjorken and S. Drell, Relativisitic Quantum Mechanics, McGraw Hill, New York, 1964. .

14. V. Budnev, et al., Phys. Rep. 15 (1975) 181

15. J. Bjorken and S. Drell, Relativisitic Quantum Mechanics, McGraw Hill, New York, 1964.

16. M. Poppe, Int. J. Mod. Phys. 1 (1986) 545

17. C. N. Yang, Jpr77 (1950) 242

18. S. Adler, Phys. Rev. 177 (1969) 2426 J.S. Bell and L. Jakiw, Nuovo Cim. 60 (1969) 47

19. D. L. Burke, R. Hollebeek, et al."Proposal to Search for Weakly-Interacting Particles using Photon Tagging", PEP Proposal 021, March 1983.

20. E. Ma and J. Okada, Phys. Rev. D18 (1978) 4219

21. G. Barbiellini, B. Richter, J. Siegrist, Phys. Lett. B106 (1981) 414

22. C. Hearty, et al., Phys. Rev. Lett. 58 (1987) 1711

23. P. Grannis, D. Jaffe and M. Marx, Nucl. Instr. and Meth. 188 (1981) 239

24. P. Levy, et al., Jour. Am. Ceram. Sos. 57 (1974) 176

25. The pulser used was similar to that described by L. R. Fortney in Proceedings of the Calorimeter Calibration Workshop,edited by H. Jostlein (Fermi National AcceleratorLaboratory, Batavia, Illinois, 1983), p. 1.

26. J. Menefee and Y. Cho, IEEE Trans. Nucl. Sci. NS-12 (1966) 159 
27. J. S. Beale, et al., Nucl. Instr. and Meth. 117 (1974) 501

28. E. Cisnero, et al., IEEE Trans. Nucl. Sci. NS-28 (1981) 465 M. Breidenbach et al., IEEE Trans. Nucl. Sci. NS-25 (1978) 706

29. For details of the ASP trigger system, see: R. J. Wilson, Proceedings of the Workshop on Triggering, Data Acquisition and Computing for High Encrgy/High Luminosity Hadron-Hadron Colliders, edited by B. Cox, R. Fenner, and P. Hale (Fermi National Accelerator Laboratory, Batavia, Illinois, 1986), p. 118.

30. R. L. Ford, W. R. Nelson, "The EGS Code System: Computer Programs for the Monte Carlo Simulation of Electromagnetic Cascade Showers (Version 3)", SLAC-PUB-0210, June 1978.

31. O.Dahl, et al., Lawrence Berkeley Laboratory, Group A Programming Note \# P-126, July 1968.

32. J. Vermaseren, Nucl. Phys. B229 (1983) 347

33. S. Brodsky and P. Lepage, Phys. Rev. D24 (1981) 1808

34. D. Karlen, Nucl. Phys. B289 (1987) 23

35. H. Cheng,E.Tsai,X.Zhu, Phys. Rev. D26 (1982) 922

36. Tony Barker and Willy Langeveld, private communication.

37. R.L. Walker, in Proceedings of the 4th International Symposium on Electron and Photon Interactions, p 36 (1969).

38. S.J. deJong, J.A.M. Vermaseren, AXO User Manual, NIKHEF-H, March 1988 (unpublished).

39. F.A. Berends, P.H. Daverveldt, and R. Kleiss, Comp. Phys. Comm. 40 (1986) 309

40. Christopher A. Hawkins, private communication.

41. For a more detailed description of the luminosity analysis, see Christopher A. Hawkins, thesis, Stanford University, 1988 (unpublished).

42. F. James and M. Roos, Comp. Phys. Comm. 10 (1975) 343 
43. D. Alde, et al., Z. Phys. C36 (1987) 603

44. D. Binnie, et al., Phys. Lett. B83 (1979) 141

45. A. Browman, et al., Phys. Rev. Lett. 32 (1974) 1067

46. A. Weinstein, et al.(Crystal Ball collaboration), Phys. Rev. D28 (1983) 2896

47. W. Bartel, et al.(JADE collaboration), Phys. Lett. B160 (1985) 421

48. H. Aihara, et al.(TPC/2 $\gamma$ collaboration), Phys. Rev. D33 (1986) 844

49. N. Roe, Mini-review of $\Gamma_{\gamma \gamma}(\eta)$, in the 1987 Review of Particle Properties, to be published.

50. G. Abrams, et al.(MarkII collaboration), Phys. Rev. Lett. 43 (1979) 477

51. P. Jenni, et al.(MarkII collaboration), Phys. Rev. D27 (1983) 1031

52. W. Bartel, et al.(JADE collaboration), Phys. Lett. B113 (1982) 190

53. H. Berend, et al.(CELLO collaboration), Phys. Lett. B114 (1982) 378

54. C. Berger, et al.(PLUTO collaboration), Phys. Lett. B142 (1984) 125

55. M. Althof, et al.(TASSO collaboration), Phys. Lett. B147 (1984) 487

56. H. Aihara, et al.(TPC/2 $\gamma$ collaboration), Phys. Rev. D35 (1987) 2650

57. H. Albrecht, et al.(ARGUS collaboration), Phys. Lett. B199 (1987) 457

58. G. Gidal, et al.(MarkII collaboration), Phys. Rev. Lett. 59 (1987) 2012

59. D. Antreasyan, et al.(Crystal Ball collaboration), Phys. Rev. D36 (1987) 2633

60. W. Bartel, et al.(JADE collaboration), Phys. Lett. B160 (1985) 421

61. J. Donoghue,B. Holstein and Y. Lin, Phys. Rev. Lett. 55 (1985) 2766

62. J. Gasser and H. Leutwyler, Nucl. Phys. B250 (1985) 465

63. F. Gilman and R. Kauffman, Phys. Rev. D36 (1987) 2761

64. A. De Rújula, H. Georgi, and S. Glashow, Phys. Rev. D12 (1975) 147 
65. J. Rosner, in Proceedings of the 1985 Inlernalional Symposium on Lepton and Photon Interactions at High Energies, Kyoto, Japan 1985, edited by M. Konuma and K. Takahashi, p. 448.

66. A. Seiden, H. Sadrozinski and H. Haber, SCIPP 87/73 (unpublished).

67. Rene Ong, private communication. 